\title{
ZERO-CYCLES ON VARIETIES OVER $p$-ADIC FIELDS AND BRAUER GROUPS
}

\author{
SHUJI SAITO AND KANETOMO SATO
}

\begin{abstract}
In this paper, we study the Brauer-Manin pairing of smooth proper varieties over local fields, and determine the $p$-adic part of the kernel of one side. We also compute the $A_{0}$ of a potentially rational surface which splits over a wildly ramified extension.
\end{abstract}

\section{INTRODUCTION}

Let $k$ be a $p$-adic local field, and let $X$ be a proper smooth geometrically integral variety over $k$. Let $\mathrm{CH}_{0}(X)$ be the Chow group of 0 -cycles on $X$ modulo rational equivalence. An important tool to study $\mathrm{CH}_{0}(X)$ is the natural pairing due to Manin [M1]

$$
\mathrm{CH}_{0}(X) \times \operatorname{Br}(X) \longrightarrow \mathbb{Q} / \mathbb{Z} \text {. }
$$

Here $\operatorname{Br}(X)$ denotes the Grothendieck-Brauer group $H_{\text {ét }}^{2}\left(X, \mathbb{G}_{\mathrm{m}}\right)$. When $\operatorname{dim}(X)=1$, using the Tate duality theorem for abelian varieties over $p$-adic local fields, Lichtenbaum [L1] proved that $(\mathbf{M})$ is non-degenerate and induces an isomorphism

$$
A_{0}(X) \stackrel{\sim}{\longrightarrow} \operatorname{Hom}(\operatorname{Br}(X) / \operatorname{Br}(k), \mathbb{Q} / \mathbb{Z}) .
$$

Here $\operatorname{Br}(X) / \operatorname{Br}(k)$ denotes the cokernel of the natural map $\operatorname{Br}(k) \rightarrow \operatorname{Br}(X)$, and $A_{0}(X)$ denotes the subgroup of $\mathrm{CH}_{0}(X)$ generated by 0 -cycles of degree 0 . An interesting question is as to whether the pairing $(\overline{\mathrm{M}})$ is non-degenerate when $\operatorname{dim}(X) \geq 2$. See [ $[\mathrm{PS}]$ for surfaces with non-zero left kernel. See [Y2] for varieties with trivial left kernel. In this paper, we are concerned with the right kernel of $(\underline{\mathrm{M}})$ in the higher-dimensional case.

1.1. We assume that $X$ has a regular model $\mathscr{X}$ which is proper flat of finite type over the integer ring $\mathfrak{o}_{k}$ of $k$. It is easy to see that the pairing $(\overline{\mathbf{M}})$ induces homomorphisms

$$
\begin{aligned}
\mathrm{CH}_{0}(X) & \longrightarrow \operatorname{Hom}(\operatorname{Br}(X) / \operatorname{Br}(\mathscr{X}), \mathbb{Q} / \mathbb{Z}), \\
A_{0}(X) & \longrightarrow \operatorname{Hom}(\operatorname{Br}(X) / \operatorname{Br}(k)+\operatorname{Br}(\mathscr{X}), \mathbb{Q} / \mathbb{Z}),
\end{aligned}
$$

where $\operatorname{Br}(X) / \operatorname{Br}(k)+\operatorname{Br}(\mathscr{X})$ denotes the quotient of $\operatorname{Br}(X)$ by the image of $\operatorname{Br}(k) \oplus$ $\operatorname{Br}(\mathscr{X})$. If $\operatorname{dim}(X)=1$, then $\operatorname{Br}(\mathscr{X})$ is zero, and the map (1.1.2) is the same as (L) (cf. [CTOP], 1.7 (c)). Our main result is the following:

Theorem 1.1.3. Assume that the purity of Brauer groups holds for $\mathscr{X}$ (Definition 1.7.1). Then:

(1) The right kernel of the pairing $(\mathrm{M})$ is exactly $\operatorname{Br}(\mathscr{X})$, that is, the map (1.1.1) has dense image with respect to the natural pro-finite topology on the right hand side.

(2) The map (1.1.2) is surjective.

Date: June 24, 2009.

2000 Mathematics Subject Classification: Primary 14C25, 14F22; Secondary 11G25, 14 G40. 
Restricted to the prime-to- $p$ part, the assertion (1) is due to Colliot-Thélène and Saito [CTS]. The assertion (2) gives an affirmative answer to [CT3], Conjecture 1.4 (c), assuming the purity of Brauer groups, which holds if $\operatorname{dim}(\mathscr{X}) \leq 3$ or if $\mathscr{X}$ has good or semistable reduction (cf. Remark 1.7.2 below). Roughly speaking, Theorem 1.1.3(1) asserts that if an element of $\omega \in \operatorname{Br}(X)$ ramifies along the closed fiber of $\mathscr{X}$, then there exists a closed point $x \in X$ such that the restriction $\omega_{x} \in \operatorname{Br}(x)$ of $\omega$ is non-zero. Using the Čebotarev density theorem, we reduce the problem to its local variant. To be more precise, we prove the following result on the ramification of Brauer groups of henselian local rings:

Theorem 1.1.4 (Corollary 3.1.2). Let $Y \subset \mathscr{X}$ be the closed fiber of $\mathscr{X} / \mathfrak{o}_{k}$ and let $U$ be a dense open regular subset of the reduced part of $Y$. Let $x$ be a closed point of $\mathscr{X}$ contained in $U$, and let $A_{x}$ be the henselization of $\mathscr{O}_{\mathscr{X}, x}$ at its maximal ideal. Let $Q_{x}$ be the set of all closed points on $\operatorname{Spec}\left(A_{x}\left[p^{-1}\right]\right)$. Assume that $k$ contains a primitive $p$-th root of unity and that the purity of Brauer groups holds for $\operatorname{Spec}(A)$. Then the map

$$
\psi_{x}: \operatorname{Br}\left(A_{x}\left[p^{-1}\right]\right) \longrightarrow \prod_{v \in Q_{x}} \mathbb{Q} / \mathbb{Z} ; \quad \omega \mapsto\left(\operatorname{inv}_{v}\left(\left.\omega\right|_{v}\right)\right)_{v \in Q_{x}}
$$

is injective.

The prime-to- $p$ part of Theorem 1.1 .4 follows from arguments in [CTS] (cf. Remark 3.4.2 below). We will prove the $p$-primary part of this local result using the computation by BlochKato on $p$-adic vanishing cycles ([BK], Corollary 1.4.1) and the Brauer group theory for 2dimensional local rings established by the first author [S1]. More precisely, because we deal with local rings which is not necessarily essentially smooth over $\mathfrak{o}_{k}$, we will generalize the Bloch-Kato theorem on $p$-adic vanishing cycles to a certain regular but non-smooth situation (see $\$ 2$ below).

1.2. As an application of Theorem 1.1.3 (2), we give an explicit calculation of $A_{0}(X)$ for a potentially rational suface $X / k$, a proper smooth geometrically connected surface $X$ over $k$ such that $\bar{X}:=X \otimes_{k} \bar{k}$ is rational. For such a surface $X$, the map (1.1.2) has been known to be injective (see Proposition 5.1.2 below), and hence bijective by Theorem 1.1.3(2). On the other hand, for such a surface $X$, we have

$$
\operatorname{Br}(X) / \operatorname{Br}(k) \simeq H_{\text {Gal }}^{1}\left(G_{k}, \mathrm{NS}(\bar{X})\right) .
$$

Here $\operatorname{NS}(\bar{X})$ denotes the Néron-Severi group of $\bar{X}$, and $G_{k}$ denotes the absolute Galois group of $X$. Thus knowing the $G_{k}$-module structure of $\operatorname{NS}(\bar{X})$, we can compute $A_{0}(X)$ by determining which element of $\operatorname{Br}(X)$ are unramified along the closed fiber of $\mathscr{X}$. For example, consider a cubic surface for $a \in k^{\times}$

$$
X: T_{0}^{3}+T_{1}^{3}+T_{2}^{3}+a T_{3}^{3}=0 \quad \text { in } \quad \mathbb{P}_{k}^{3}=\operatorname{Proj}\left(k\left[T_{0}, T_{1}, T_{2}, T_{3}\right]\right) .
$$

If $a$ is a cube in $k$, then $X$ is isomorphic to the blow-up of $\mathbb{P}_{k}^{2}$ at six $k$-valued points in the general position (Shafarevich) and we have $A_{0}(X)=0$. We will prove the following result, which is an extention of results in [CTS], Example 2.8.

Theorem 1.2.1 (Theorem 5.1.1). Assume that $\operatorname{ord}_{k}(a) \equiv 1 \bmod (3)$ and that $k$ contains a primitive cubic root of unity. Then we have

$$
A_{0}(X) \simeq(\mathbb{Z} / 3)^{2}
$$


In his paper [Da], Dalawat provided a method to compute $A_{0}(X)$ for a potentially rational surface $X$, which works under the assumption that the action of $G_{k}$ on $\operatorname{NS}(\bar{X})$ is unramified. Theorem 1.1.3 provides a new method to compute $A_{0}(X)$, which does not requires Dalawat's assumption. Note that $p$ may be 3 in Theorem 1.2.1, so that the action of $G_{k}$ on $\operatorname{NS}(\bar{X})$ may ramify even wildly.

1.3. Let $\mathfrak{o}_{k}$ be as before, and let $\mathscr{X}$ be a regular scheme which is proper flat of finite type over $\mathfrak{o}_{k}$. Assume that $\mathscr{X}$ has good or semistable reduction over $\mathfrak{o}_{k}$. Let $d$ be the absolute dimension of $\mathscr{X}$, and let $r$ be a positive integer. In [SS1], we proved that the cycle class map

$$
\varrho_{m}^{d-1}: \mathrm{CH}^{d-1}(\mathscr{X}) / m \longrightarrow H_{\text {ét }}^{2 d-2}\left(\mathscr{X}, \mu_{m}^{\otimes d-1}\right)
$$

is bijective for any positive integer $m$ prime to $p$. Here $\mu_{m}$ denotes the étale sheaf of $m$-th roots of unity. As a new tool to study $\mathrm{CH}^{d-1}(\mathscr{X})$, we introduce the $p$-adic cycle class map defined in [Sa2], Corollary 6.1.4:

$$
\varrho_{p^{r}}^{d-1}: \mathrm{CH}^{d-1}(\mathscr{X}) / p^{r} \longrightarrow H_{\text {ét }}^{2 d-2}\left(\mathscr{X}, \mathfrak{T}_{r}(d-1)\right) .
$$

Here $\mathfrak{T}_{r}(n)=\mathfrak{T}_{r}(n)_{\mathscr{X}}$ denotes the étale Tate twist with $\mathbb{Z} / p^{r} \mathbb{Z}$-coefficients [Sa2] (see also $[\mathrm{Sch}], \S 7)$, which is an object of $D^{b}\left(\mathscr{X}, \mathbb{Z} / p^{r} \mathbb{Z}\right)$, the derived category of bounded complexes of étale $\mathbb{Z} / p^{r} \mathbb{Z}$-sheaves on $\mathscr{X}$. This object $\mathfrak{T}_{r}(n)$ plays the role of $\mu_{m}^{\otimes n}$, and we expect that $\mathfrak{T}_{r}(n)$ agrees with $\mathbb{Z}(n)^{\text {ét }} \otimes \mathbb{L} \mathbb{Z} / p^{r} \mathbb{Z}$, where $\mathbb{Z}(n)^{\text {ét }}$ denotes the conjectural étale motivic

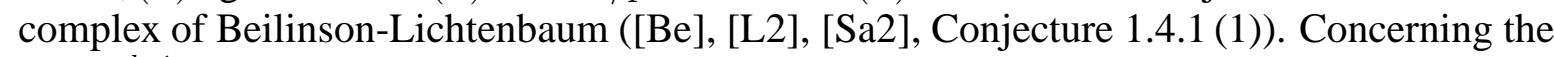
map $\varrho_{p^{r}}^{d-1}$, we will prove the following result:

Theorem 1.3.1. The cycle class map $\varrho_{p^{r}}^{d-1}$ is surjective.

We have nothing to say about the injectivity of $\varrho_{p^{r}}^{d-1}$ in this paper (compare with [YY1]). A key to the proof of Theorem 1.3 .1 is the non-degeneracy of a canonical pairing of finite $\mathbb{Z} / p^{r} \mathbb{Z}$ modules

$$
H_{\text {ét }}^{2 d-2}\left(\mathscr{X}, \mathfrak{T}_{r}(d-1)\right) \times H_{Y, \text { ét }}^{3}\left(\mathscr{X}, \mathfrak{T}_{r}(1)\right) \longrightarrow \mathbb{Z} / p^{r} \mathbb{Z}
$$

proved in [Sa2], Theorem 10.1.1. We explain an outline of the proof of Theorem 1.3.1, assuming that $k$ contains a primitive $p$-th root of unity. Let $Y, U, A_{x}$ be as in Theorem 1.1.4. Let $X_{0}$ and $Y_{0}$ be the sets of all closed points on $X$ and $Y$, respectively, and let sp : $X_{0} \rightarrow Y_{0}$ be the specialization map of points. By the duality mentioned above, there is an isomorphism of finite groups

$$
H_{\text {ét }}^{2 d-2}\left(\mathscr{X}, \mathfrak{T}_{r}(d-1)\right) \stackrel{\sim}{\longrightarrow} H_{Y \text {,ét }}^{3}\left(\mathscr{X}, \mathfrak{T}_{r}(1)\right)^{*},
$$

where we put $M^{*}:=\operatorname{Hom}(M, \mathbb{Q} / \mathbb{Z})$ for abelian group $M$. We will construct an injective map

$$
\theta_{p^{r}}: H_{Y, \text { ét }}^{3}\left(\mathscr{X}, \mathfrak{T}_{r}(1)\right) \hookrightarrow \prod_{x \in U_{0}} p^{r} \operatorname{Br}\left(A_{x}\left[p^{-1}\right]\right)
$$

whose dual fits into a commutative diagram

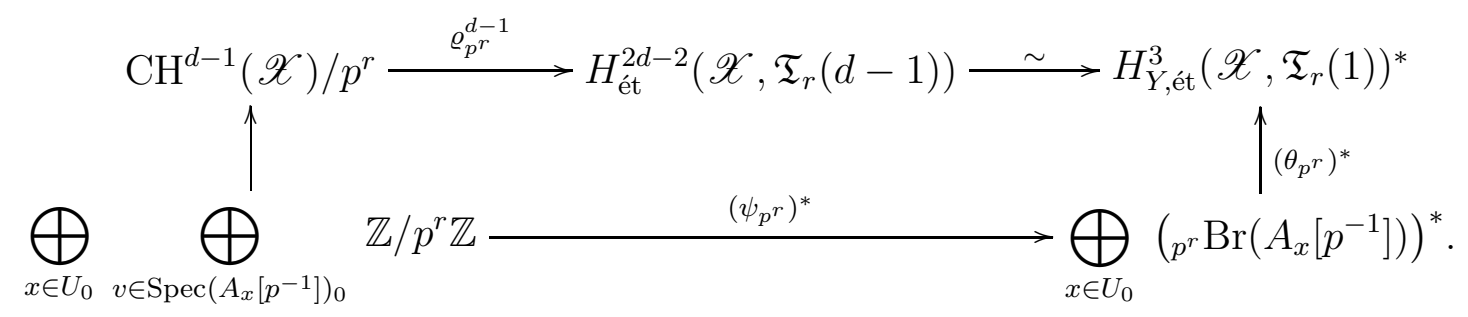


Here $\psi_{p^{r}}$ denotes the direct product of the $p^{r}$-torsion part of the map $\psi_{x}$ in Theorem 1.1.4 for all $x \in U_{0}$, which is injective by Theorem 1.1.4. Thus Theorem 1.3.1 will follow from this commutative diagram and the surjectivity of $\left(\theta_{p^{r}}\right)^{*}$ and $\left(\psi_{p^{r}}\right)^{*}$ (see $\oint 7$ for details).

1.4. This paper is organized as follows. In $\S 2$, we will study the structure of Brauer groups of certain regular local rings, using the computation on étale sheaves of $p$-adic vanishing cycles originally due to Bloch-Kato $[\overline{\mathrm{BK}}]$. In $\S 3$, we will prove the key local result Theorem 1.1.4 (see Remark 3.4.2 for the prime-to- $p$ part). In \$4, we will establish an unramified theory for Brauer groups of varieties over $p$-adic fields (cf. Theorem 4.2.1, Corollary 4.2.3). In $₫ 5$, we compute $A_{0}$ of cubic surfaces to prove Theorem 1.2.1, In $₫ 6$ and $\S 7$, we will prove Theorem 1.1.3 and Theorem 1.3.1, respectively.

Acknowledgements. The research for this article was partially supported by EPSRC grant and JSPS Core-to-Core Program. The authors express their gratitude to Professor Ivan Fesenko for valuable comments and discussions and to The University of Nottingham for their great hospitality. Thanks are also due to Professor Jean-Louis Colliot-Thélène for helpful conversations and allowing them to include his note [CT4] in this paper.

\section{NOTATION}

1.5. For an abelian group $M$ and a positive integer $n,{ }_{n} M$ and $M / n$ denote the kernel and the cokernel of the map $M \stackrel{\times n}{\longrightarrow} M$, respectively. For a field $k, \bar{k}$ denotes a fixed separable closure, and $G_{k}$ denotes the absolute Galois group $\operatorname{Gal}(\bar{k} / k)$. For a discrete $G_{k}$-module $M$, $H^{*}(k, M)$ denote the Galois cohomology groups $H_{\mathrm{Gal}}^{*}\left(G_{k}, M\right)$, which are the same as the étale cohomology groups of $\operatorname{Spec}(k)$ with coefficients in the étale sheaf associated with $M$.

1.6. Unless indicated otherwise, all cohomology groups of schemes are taken over the étale topology. For a commutatitive ring $R$ with unity and a sheaf $\mathscr{F}$ on $\operatorname{Spec}(R)_{\text {ét }}$, we often write $H^{*}(R, \mathscr{F})$ for $H^{*}(\operatorname{Spec}(R), \mathscr{F})$.

1.7. For a scheme $X$, a sheaf $\mathscr{F}$ on $X_{\text {ét }}$ and a point $x \in X$, we often write $H_{x}^{*}(X, \mathscr{F})$ for $H_{x}^{*}\left(\operatorname{Spec}\left(\mathscr{O}_{X, x}\right), \mathscr{F}\right)$. For a point $x \in X, \kappa(x)$ denotes its residue field. We often write $X_{0}$ for the set of all closed points on $X$. For a pure-dimensional scheme $X$ and a non-negative integer $q, X^{q}$ denotes the set of all points on $X$ of codimension $q$. For an integer $n \geq 0$ and a noetherian excellent scheme $X, \mathrm{CH}_{n}(X)$ denotes the Chow group of algebraic cycles on $X$ of dimension $n$ modulo rational equivalence; if $X$ is regular of pure dimension $d$, we often write $\mathrm{CH}^{n}(X)$ for $\mathrm{CH}_{d-n}(X)$. Finally we introduce the following terminology:

Definition 1.7.1. Let $X$ be a noetherian regular scheme.

(1) For a closed immersion $\iota_{Z}: Z \rightarrow X$ with $\operatorname{codim}_{X}(Z) \geq 2$, we say that the purity of Brauer groups holds for the pair $(X, Z)$, if $R^{3} \iota_{Z}^{!} \mathbb{G}_{\mathrm{m}, X}=0$.

(2) We say that the purity of Brauer groups holds for $X$, if the purity of Brauer groups holds for any pair $(X, Z)$ with $\operatorname{codim}_{X}(Z) \geq 2$.

Remark 1.7.2. There are some known cases on this purity problem:

(1) For a noetherian regular scheme $X$ with $\operatorname{dim}(X) \leq 3$, the purity of Brauer groups holds for $X([\overline{\mathrm{Ga}}])$. 
(2) For a noetherian regular scheme $X$ and a prime number $\ell$ invertible on $X$, the purity of Brauer groups holds for $X$ with respect to the $\ell$-primary torsion part ([RZ], [Th], $[\mathrm{F}])$.

(3) For a regular scheme $X$ over $\mathbb{F}_{p}$, the purity of Brauer groups holds for $X$ with respect

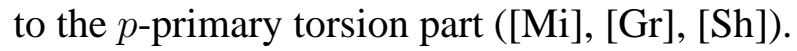

(4) Let $p$ be a prime number, and let $k$ be a henselian discrete valuation field of characteristic zero whose residue field is perfect of characteristic $p$. Then for a smooth or semistable family $X$ over the integer ring of $k$, the purity of Brauer groups holds for $X$ with respect to the $p$-primary torsion part ([Sa2], Corollary 4.5.2). This purity fact is a consequence of a result of Hagihara [Sa2], Theorem A.2.6, which relies on the Bloch-Kato-Hyodo theorem on étale sheaves of $p$-adic vanishing cycles $([\overline{\mathrm{BK}}],[\mathrm{H}])$. 


\section{Structure of Brauer GRoups of LOCAL RingS}

Let $p$ be a prime number, and let $k$ be a henselian discrete valuation field of characteristic 0 whose residue field $\mathbb{F}$ is finite and has characteristic $p$. Let $\mathfrak{o}_{k}$ be the integer ring of $k$.

2.1. Setting. Let $\mathscr{X}$ be a noetherian integral regular scheme of dimension at least 2 which is faithfully flat of finite type over $S:=\operatorname{Spec}\left(\mathfrak{o}_{k}\right)$. Let $Y$ be the divisor on $\mathscr{X}$ defined by the radical of $(p) \subset \mathscr{O}_{\mathscr{X}}$. We assume that $k$ contains a primitive $p$-th root of unity $\zeta_{p}$ and that $\mathscr{X}$ satisfies the following condition:

(2.1.1) The divisor $Y \subset \mathscr{X}$ is connected, principal and smooth over $\operatorname{Spec}(\mathbb{F})$.

Note that the generic fiber $X=\mathscr{X} \otimes_{\mathfrak{o}_{k}} k$ is smooth over $\operatorname{Spec}(k)$, because $k$ is perfect. Fix a uniformizer $T$ of $Y$ on $\mathscr{X}$. Throughout this section, we always work in this setting.

2.2. Impure part of Brauer groups. Let $\iota$ and $j$ be as follows:

$$
Y \stackrel{\iota}{\longrightarrow} \mathscr{X} \stackrel{j}{\longleftarrow} X \text {. }
$$

For a point $y$ on $Y$, let $i_{y}$ be the canonical map $y \rightarrow Y$. Put $\mathbb{G}_{\mathrm{m}}:=\mathscr{O}_{\mathscr{X}}^{\times}$. In this section, we are concerned with the following two étale sheaves on $Y$ :

$$
R^{3} \iota^{!} \mathbb{G}_{\mathrm{m}}\left(\simeq \iota^{*} R^{2} j_{*} \mathscr{O}_{X}^{\times}\right) \quad \text { and } \quad M^{2}:=\iota^{*} R^{2} j_{*} \mu_{p}^{\otimes 2} .
$$

The first result of this section will be stated in the next subsection (see Theorem 2.3.3 below). Since $\mathscr{X}$ is regular, $R^{3} \iota^{!} \mathbb{G}_{\mathrm{m}}$ is a torsion sheaf. We define the impure part $\mathscr{I}$ of Brauer groups as follows:

$$
\mathscr{I}:=\operatorname{Ker}\left(R^{3} \iota^{!} \mathbb{G}_{\mathrm{m}} \rightarrow \bigoplus_{\eta \in Y^{0}} R^{3} i_{\eta *}\left(i_{\eta}^{*} R \iota ! \mathbb{G}_{\mathrm{m}}\right)\right),
$$

where for $y \in Y, i_{y}$ denotes the canonical map $y \rightarrow Y$. In fact, the following lemma holds:

Lemma 2.2.1. For a closed immersion $i_{Z}: Z \rightarrow Y$ with $\iota_{Z}: Z \rightarrow Y \rightarrow \mathscr{X}$, the natural map $i_{Z *} R^{3} \iota_{Z} \mathbb{G}_{\mathrm{m}} \rightarrow R^{3} \iota^{!} \mathbb{G}_{\mathrm{m}}$ is injective, and we have

$$
\mathscr{I}=\bigcup_{Z \subset Y, \operatorname{codim}_{Y}(Z) \geq 1} \operatorname{Im}\left(i_{Z *} R^{3} \iota_{Z} \mathbb{G}_{\mathrm{m}} \rightarrow R^{3} \iota^{!} \mathbb{G}_{\mathrm{m}}\right),
$$

where $Z$ runs through all closed subschemes of $Y$ of codimension $\geq 1$ in $Y$. Consequently, the purity of Brauer groups holds for $\mathscr{X}$ if and only if $\mathscr{I}=0$.

Proof. Let $i_{Z}: Z \rightarrow Y$ be a closed immersion, and let $\psi$ be the open immersion $Y \backslash Z \rightarrow Y$. Let $\iota_{Z}$ be the composite map $Z \rightarrow Y \rightarrow \mathscr{X}$. We have the following localization exact sequence of sheaves on $Y_{\text {ét }}$ :

$$
R^{2} \psi_{*}\left(\psi^{*} R \iota^{!} \mathbb{G}_{\mathrm{m}}\right) \longrightarrow i_{Z *} R^{3} \iota_{Z}^{!} \mathbb{G}_{\mathrm{m}} \longrightarrow R^{3} \iota^{!} \mathbb{G}_{\mathrm{m}} \longrightarrow R^{3} \psi_{*}\left(\psi^{*} R \iota^{!} \mathbb{G}_{\mathrm{m}}\right) .
$$

To show the first assertion, we show that $R^{2} \psi_{*}\left(\psi^{*} R \iota^{!} \mathbb{G}_{\mathrm{m}}\right)$ is the zero sheaf. By the standard purity for $\mathbb{G}_{\mathrm{m}}$ in $[\mathrm{G}]$, III.6, we have

$$
R^{0} \iota^{!} \mathbb{G}_{\mathrm{m}}=0=R^{2} \iota^{!} \mathbb{G}_{\mathrm{m}} \text { and } \quad R^{1} \iota ! \mathbb{G}_{\mathrm{m}} \simeq \mathbb{Z}_{Y},
$$

and hence, $R^{2} \psi_{*}\left(\psi^{*} R \iota^{!} \mathbb{G}_{\mathrm{m}}\right) \simeq R^{1} \psi_{*} \mathbb{Z}_{Y \backslash Z}=0$. The second assertion follows from the exact sequence (2.2.2) and a standard limit argument. 
2.3. Structure of $M^{2}$. We define the sheaf $I$ on $Y_{\text {ét }}$ as follows:

$$
I:={ }_{p} \mathscr{I}=\operatorname{Ker}(\mathscr{I} \stackrel{\times p}{\longrightarrow} \mathscr{I}) .
$$

The sheaf $I \otimes \iota^{*} j_{*} \mu_{p}$ is regarded as a subsheaf of $M^{2}$ in the following canonical way. We have $R^{3} \iota^{!} \mathbb{G}_{\mathrm{m}} \simeq \iota^{*} R^{2} j_{*} \mathscr{O}_{X}^{\times}$and the following exact sequence:

$$
(0=) \iota^{*} R^{1} j_{*} \mathscr{O}_{X}^{\times} \longrightarrow \iota^{*} R^{2} j_{*} \mu_{p} \longrightarrow \iota^{*} R^{2} j_{*} \mathscr{O}_{X}^{\times} \stackrel{\times p}{\longrightarrow} \iota^{*} R^{2} j_{*} \mathscr{O}_{X}^{\times},
$$

where $\iota^{*} R^{1} j_{*} \mathscr{O}_{X}^{\times}$is zero because $\mathscr{O}_{\mathscr{X}, \bar{x}}^{\mathrm{sh}}\left[p^{-1}\right]$ is a UFD for any $x \in Y$. Hence we have

$$
I \otimes \iota^{*} j_{*} \mu_{p} \subset M^{2}
$$

by the isomorphism $\iota^{*} R^{2} j_{*} \mu_{p} \otimes \iota^{*} j_{*} \mu_{p} \simeq M^{2}$ (since $\zeta_{p} \in k$ ). We next define the descending filtration $U^{\bullet}$ on $M^{2}$ of different arithmetic nature as follows.

Definition 2.3.2. (1) We define the Milnor $K$-sheaf $\mathscr{K}_{2, X / Y}^{M}$ on $Y_{\text {ét }}$ as

$$
\mathscr{K}_{2, X / Y}^{M}:=\left(\iota^{*} j_{*} \mathscr{O}_{X}^{\times}\right)^{\otimes 2} / \mathfrak{J},
$$

where $\mathfrak{J}$ denotes the subsheaf of $\left(\iota^{*} j_{*} \mathscr{O}_{X}^{\times}\right)^{\otimes 2}$ generated by local sections

$$
f \otimes g \quad \text { with } f, g \in \iota^{*} j_{*} \mathscr{O}_{X}^{\times} \text {and } f+g=0 \text { or } 1 .
$$

In what follows, we put $\mathscr{K}_{2}^{M}:=\mathscr{K}_{2, X / Y}^{M}$ for simplicity.

(2) We define the étale subsheaf $U^{m} \mathscr{K}_{2}^{M} \subset \mathscr{K}_{2}^{M}(m \geq 0)$ as the full sheaf $\mathscr{K}_{2}^{M}$ if $m=0$, and as the subsheaf generated by local sections of the form

$$
\left\{1+f \cdot T^{m}, g\right\} \quad \text { with } f \in \iota^{*} \mathscr{O}_{\mathscr{X}} \text { and } g \in \iota^{*} j_{*} \mathscr{O}_{X}^{\times}
$$

if $m \geq 1$, where $T$ is the uniformizer of $Y \subset \mathscr{X}$ fixed in $\$ 2.1$.

(3) We define the filtration $U^{m}(m \geq 0)$ on $M^{2}$ as the image of $U^{m} \mathscr{K}_{2}^{M}$ under the symbol map $\mathscr{K}_{2}^{M} \rightarrow M^{2}$ of Tate-Bloch-Kato [BK], (1.2).

Let $e$ be the absolute ramification index of $\mathscr{X}$ along $Y$, that is, the order of $p$ with respect to $T$. Put $e^{\prime}:=p e /(p-1)$, which is a positive integer divided by $p$ by the assumption that $\zeta_{p} \in k$. The first result of this section is the following:

Theorem 2.3.3. (1) We have the following canonical direct decomposition on $Y_{\text {ét }}$ :

$$
M^{2} \stackrel{\sim}{\sim} U^{0} M^{2} \oplus\left(I \otimes \iota^{*} j_{*} \mu_{p}\right) .
$$

(2) We have $U^{e^{\prime}} M^{2}=0$, and for an integer $m$ with $0 \leq m<e^{\prime}$, we have the isomorphism

$$
\operatorname{gr}_{U}^{m} M^{2}\left(:=U^{m} M^{2} / U^{m+1} M^{2}\right) \simeq \begin{cases}\Omega_{Y, \log }^{2} \oplus \Omega_{Y, \log }^{1} & (m=0) \\ \Omega_{Y}^{1} & \left(0<m<e^{\prime}, p \nmid m\right) \\ \Omega_{Y}^{1} / \mathscr{Z}_{Y}^{1} \oplus \mathscr{O}_{Y} /\left(\mathscr{O}_{Y}\right)^{p} & \left(0<m<e^{\prime}, p \mid m\right)\end{cases}
$$

given by the following, respectively:

$$
\begin{aligned}
\left\{g_{1}, g_{2}\right\}+\left\{T, g_{3}\right\} & \mapsto\left(d \log \left(\overline{g_{1}}\right) \wedge d \log \left(\overline{g_{2}}\right), d \log \left(\overline{g_{3}}\right)\right) \\
\left\{1+f_{1} \cdot T^{m}, g_{1}\right\}+\left\{1+f_{2} \cdot T^{m}, T\right\} & \mapsto \overline{f_{1}} \cdot d \log \left(\overline{g_{1}}\right)-m^{-1} \cdot d f_{2} \\
\left\{1+f_{1} \cdot T^{m}, g_{1}\right\}+\left\{1+f_{2} \cdot T^{m}, T\right\} & \mapsto\left(\overline{f_{1}} \cdot d \log \left(\overline{g_{1}}\right), \overline{f_{2}}\right),
\end{aligned}
$$


where $g_{\lambda}$ 's (resp. $f_{\lambda}$ 's) are local sections of $\iota^{*} \mathbb{G}_{\mathrm{m}}\left(\right.$ resp. $\left.\iota^{*} \mathscr{O}_{\mathscr{X}}\right)$, and $\overline{g_{\lambda}}\left(\right.$ resp. $\left.\overline{f_{\lambda}}\right)$ denotes the residue class of $g_{\lambda}$ in $\mathscr{O}_{Y}^{\times}$(resp. the residue class of $f_{\lambda}$ in $\left.\mathscr{O}_{Y}\right)$. We defined $\mathscr{Z}_{Y}^{1}$ as the kernel of $d: \Omega_{Y}^{1} \rightarrow \Omega_{Y}^{2}$.

This theorem is due to Bloch-Kato if $\mathscr{X}$ is smooth over $S$ (cf. [BK], Theorem (1.4)). Our result is a generalization of their arguments to the situation in (2.1.1). Using Theorem 2.3.3, we will prove Theorem 2.6.2 below, which will play important roles in this paper. We prove Theorem 2.3.3 in $\S \S 2.42 .5$.

Remark 2.3.5. In our proof of Theorem 2.3.3, we will use the Bloch-Kato theorem for strict henselian discrete valuation fields $[\overline{\mathrm{BK}}]$, Theorem (5.12) and the Merkur'ev-Suslin theorem [MS] for fraction fields of strict local rings of $\mathscr{X}$ at points of codimension 2 (see Lemma 2.4.7 below).

2.4. Preliminary on Theorem 2.3.3. We prove two lemmas (Lemmas 2.4.1 and 2.4.7), which will play key roles in our proof of Theorem 2.3.3. We first show the following:

Lemma 2.4.1. Put $L^{2}:=\mathscr{K}_{2}^{M} / p \mathscr{K}_{2}^{M}$, and define the filtration $U^{\bullet}$ on $L^{2}$ as the image of $U^{\bullet} \mathscr{K}_{2}^{M}$. Then the following holds:

(1) We have $U^{e^{\prime}} L^{2}=0$.

(2) For $m$ with $0 \leq m<e^{\prime}$, there is a well-defined surjective homomorphism

$$
\varrho_{m}:\left\{\begin{array}{ll}
\Omega_{Y, \log }^{2} \oplus \Omega_{Y, \log }^{1} & (m=0) \\
\Omega_{Y}^{1} & \left(0<m<e^{\prime}, p \nmid m\right) \\
\Omega_{Y}^{1} / \mathscr{Z}_{Y}^{1} \oplus \mathscr{O}_{Y} /\left(\mathscr{O}_{Y}\right)^{p} & \left(0<m<e^{\prime}, p \mid m\right)
\end{array}\right\} \longrightarrow \operatorname{gr}_{U}^{m} L^{2}
$$

given by

$$
\begin{aligned}
\left(d \log \left(g_{1}\right) \wedge d \log \left(g_{2}\right), d \log \left(g_{3}\right)\right) & \mapsto\left\{\widetilde{g_{1}}, \widetilde{g}_{2}\right\}+\left\{T, \widetilde{g}_{3}\right\} \\
f_{1} \cdot d \log \left(g_{1}\right) & \mapsto\left\{1+\widetilde{f_{1}} \cdot T^{m}, \widetilde{g}_{1}\right\} \\
\left(f_{1} \cdot d \log \left(g_{1}\right), f_{2}\right) & \mapsto\left\{1+\widetilde{f_{1}} \cdot T^{m}, \widetilde{g}_{1}\right\}+\left\{1+\widetilde{f_{2}} \cdot T^{m}, T\right\},
\end{aligned}
$$

respectively, where $g_{\lambda}$ 's (resp. $f_{\lambda}$ 's) are local sections of $\mathscr{O}_{Y}^{\times}$(resp. of $\left.\mathscr{O}_{Y}\right)$, and $\widetilde{g_{\lambda}}$ (resp. $\left.\tilde{f}_{\lambda}\right)$ denotes a lift of $g_{\lambda}$ to $\iota^{*} \mathbb{G}_{\mathrm{m}}$ (resp. a lift of $f_{\lambda}$ to $\left.\iota^{*} \mathscr{O}_{\mathscr{X}}\right)$ for each $\lambda$.

Proof. (1) For $y \in Y$ and $f \in \mathscr{O}_{\mathscr{X}, \bar{y}}^{\mathrm{sh}}$, there exists $g \in \mathscr{O}_{\mathscr{X}, \bar{y}}^{\mathrm{sh}}$ satisfying

$$
1+f \cdot T^{e^{\prime}}=\left(1+g \cdot T^{e /(p-1)}\right)^{p},
$$

because $\mathscr{O}_{\mathscr{X}, \bar{y}}^{\mathrm{sh}}$ is strictly henselian. Hence we have $U^{e^{\prime}} L^{2}=0$.

(2) The well-definedness and the surjectivity of $\varrho_{m}$ for $m \geq 1$ is due to Bloch-Kato [BK], (4.3), Lemma (4.5), (4.7). We prove the well-definedness and the surjectivity of $\varrho_{0}$. We define the sheaf $\mathscr{K}_{2, Y}^{M}$ on $Y_{\text {ét }}$ as

$$
\mathscr{K}_{2, Y}^{M}:=\left(\mathbb{G}_{\mathrm{m}, Y}\right)^{\otimes 2} / \mathfrak{J}^{\prime}
$$

where $\mathfrak{J}^{\prime}$ denotes the subsheaf of $\left(\mathbb{G}_{\mathrm{m}}, Y\right)^{\otimes 2}$ generated by local sections

$$
f \otimes g \quad \text { with } f, g \in \mathbb{G}_{\mathrm{m}, Y} \text { and } f+g=0 \text { or } 1 .
$$

We define the homomorphism

$$
\sigma: \mathscr{K}_{2, Y}^{M} \oplus \mathbb{G}_{\mathrm{m}, Y} \longrightarrow \operatorname{gr}_{U}^{0} \mathscr{K}_{2}^{M}
$$


of sheaves on $Y_{\text {ét }}$ as

$$
\left(\left\{g_{1}, g_{2}\right\}, g_{3}\right) \mapsto\left\{\widetilde{g_{1}}, \widetilde{g_{2}}\right\}+\left\{T, \widetilde{g_{3}}\right\}+U^{1} \mathscr{K}_{2}^{M},
$$

where $g_{\lambda}$ 's and $\widetilde{g_{\lambda}}$ 's are the same as those in Lemma 2.4.1 (1). One can easily check that $\sigma$ is well-defined and surjective by an elementary computation on symbols. Now $\sigma$ induces the map

$$
\mathscr{K}_{2, Y}^{M} / p \mathscr{K}_{2, Y}^{M} \oplus \Omega_{Y, \log }^{1} \longrightarrow \operatorname{gr}_{U}^{0} L^{2}
$$

which is surjective as well. Thus we are reduced to the following lemma:

Lemma 2.4.4. The differential symbol map dlog : $\mathscr{K}_{2, Y}^{M} / p \mathscr{K}_{2, Y}^{M} \rightarrow \Omega_{Y, \log }^{2}$ is bijective on $Y_{\text {ét. }}$

Proof of Lemma 2.4.4 Let $y$ be an arbitrary point on $Y$ and let $R$ be the strict henselization of the local ring $\mathscr{O}_{Y, y}$ at its maximal ideal. Our task is to show that the map of stalks at $\bar{y}$ :

$$
d \log : K_{2}^{M}(R) / p K_{2}^{M}(R) \longrightarrow \Omega_{R, \log }^{2}
$$

is bijective. This follows from a theorem of Bloch-Gabber-Kato [BK] Theorem (2.1) (see also [CTSS], p. 777, Théorème) and the following classical result due to Stein, Matsumoto, and van der Kallen (cf. [ $[\mathrm{Sr}]$, Theorem 1.14, p. 17, Remark):

Lemma 2.4.5. Let $B$ be a local ring. Then the Steinberg symbol map

$$
s: K_{2}^{M}(B) \longrightarrow K_{2}(B)
$$

is surjective, which is bijective if $B$ is a field or the residue field of $B$ has at least 5 elements. This completes the proof of Lemma 2.4.4 and Lemma 2.4.1.

Remark 2.4.6. The map (2.4.2) is bijective. Indeed, one can check the injectivity by the section

$$
\begin{gathered}
\tau: \operatorname{gr}_{U}^{0} \mathscr{K}_{2}^{M} \longrightarrow \mathscr{K}_{2, Y}^{M} \oplus \mathbb{G}_{\mathrm{m}}, Y \\
\left\{g_{1}, g_{2}\right\}+\left\{T, g_{3}\right\}+U^{1} \mathscr{K}_{2}^{M} \longmapsto\left(\left\{\overline{g_{1}}, \overline{g_{2}}\right\}, \overline{g_{3}}\right),
\end{gathered}
$$

where $g_{\lambda}$ 's and $\overline{g_{\lambda}}$ 's are the same as in Theorem 2.3.3(2). Consequently, (2.4.3) is also bijective.

Lemma 2.4.7. We have $\left(U^{0} M^{2}\right)_{\bar{y}}=\left(M^{2}\right)_{\bar{y}}$ for any point $y \in Y$ with $\operatorname{codim}_{Y}(y) \leq 1$.

Proof. The case $y \in Y^{0}$ is due to Bloch-Kato $[\mathrm{BK}]$, Theorem (5.12). Let $y$ be a point in $Y^{1}$, and put $A:=\mathscr{O}_{\mathscr{X}, \bar{y}}^{\mathrm{sh}}$. Let $K$ be the fraction field of $A$. Our task is to show that the symbol map

$$
K_{2}^{M}\left(A\left[p^{-1}\right]\right) \longrightarrow H^{2}\left(A\left[p^{-1}\right], \mu_{p}^{\otimes 2}\right)
$$

is surjective. We prove that this map naturally factors through the Steinberg symbol map:

$$
K_{2}^{M}\left(A\left[p^{-1}\right]\right) \stackrel{s}{\longrightarrow} K_{2}\left(A\left[p^{-1}\right]\right) \stackrel{t}{\longrightarrow} H^{2}\left(A\left[p^{-1}\right], \mu_{p}^{\otimes 2}\right),
$$

and that $t$ is surjective; the surjectivity of $s$ is an easy exercise using Lemma 2.4.5 and left to the reader. Since $A\left[p^{-1}\right]$ is a PID, we have the following exact sequence coming from the localization sequence of algebraic $K$-groups:

$$
K_{2}\left(A\left[p^{-1}\right]\right) \longrightarrow K_{2}^{M}(K) \stackrel{\delta}{\longrightarrow} \bigoplus_{v \in Q} \kappa(v)^{\times} \longrightarrow 0
$$


where $Q$ denotes the set of all closed points on $\operatorname{Spec}\left(A\left[p^{-1}\right]\right)$ and $\delta$ is given by tame symbol maps. Now consider the following commutative diagram with exact rows:

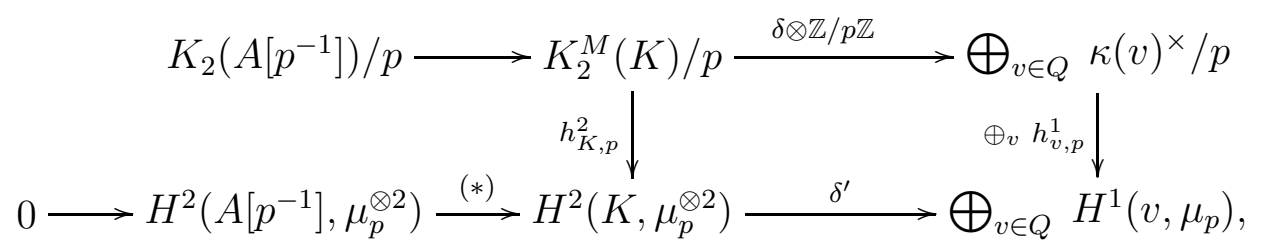

where $\delta^{\prime}$ denotes the boundary map of Galois cohomology groups, and the exactness of the lower row follows from the smooth purity in characteristic 0 . The injectivity of $(*)$ follows from the fact that $A\left[p^{-1}\right]$ is a PID containing a primitive $p$-th root of unity. The vertical maps are Galois symbol maps (cf. $[\bar{K}]$, p. 608, see also loc. cit., p. 610, Lemma 1 for the commutativity). By this diagram, we obtain the map $t$ in (2.4.9) decomposing (2.4.8). Finally, $t$ is surjective, because $\oplus_{v} h_{v, p}^{1}$ is injective and $h_{K, p}^{2}$ is bijective by the Merkur'ev-Suslin theorem [MS]. Thus we obtain Lemma 2.4.7.

2.5. Proof of Theorem 2.3.3. We begin the proof of Theorem 2.3.3. Let $\eta$ be the generic point of $Y$, and let $K_{\bar{\eta}}$ be the fraction field of $\mathscr{O}_{\mathscr{X}, \bar{\eta}}^{\mathrm{sh}}$. We first show Theorem 2.3.3(2). Note that $U^{e^{\prime}} M^{2}=0$ by Lemma2.4.1(1). By Lemma 2.4.1)(2) and [BK], Lemmas (5.2), (5.3) for $K_{\bar{\eta}}$, we have the following composite maps of sheaves for $0 \leq m<e^{\prime}$ :

$$
\begin{array}{r}
\left.\Omega_{Y, \log }^{2} \oplus \Omega_{Y, \log }^{1} \stackrel{\varrho_{m}}{\longrightarrow} \operatorname{gr}_{U}^{m} M^{2} \longrightarrow i_{\eta *} i_{\eta}^{*} \operatorname{gr}_{U}^{m} M^{2} \simeq i_{\eta *}\left(\Omega_{\eta, \log }^{2} \oplus \Omega_{\eta, \log }^{1}\right) \quad \text { (if } m=0\right), \\
\left.\Omega_{Y}^{1} \stackrel{\varrho_{m}}{\longrightarrow} \operatorname{gr}_{U}^{m} M^{2} \longrightarrow i_{\eta *} i_{\eta}^{*} \operatorname{gr}_{U}^{m} M^{2} \simeq i_{\eta *} \Omega_{\eta}^{1} \quad \text { (if } p \nmid m\right), \\
\Omega_{Y}^{1} / \mathscr{Z}_{Y}^{1} \oplus \mathscr{O}_{Y} /\left(\mathscr{O}_{Y}\right)^{p} \stackrel{\varrho_{m}}{\longrightarrow} \operatorname{gr}_{U}^{m} M^{2} \longrightarrow i_{\eta *} i_{\eta}^{*} \operatorname{gr}_{U}^{m} M^{2} \simeq i_{\eta *}\left(\Omega_{\eta}^{1} / \mathscr{Z}_{\eta}^{1} \oplus \mathscr{O}_{\eta} /\left(\mathscr{O}_{\eta}\right)^{p}\right) \\
\quad \text { (if } m \neq 0, p \mid m) .
\end{array}
$$

These maps are injective by the smoothness of $Y$. Hence we obtain Theorem 2.3.3 (2) by the surjectivity of $\varrho_{m}$. Next we prove Theorem 2.3.3(1). Put

$$
J:=\operatorname{Ker}\left(M^{2} \rightarrow i_{\eta *} i_{\eta}^{*} M^{2}\right) .
$$

By the proof of Theorem 2.3.3 (2), we have

$$
U^{0} M^{2} \cap J=0 .
$$

The problem is reduced to the following:

Proposition 2.5.2. (1) $M^{2}=U^{0} M^{2}+J$.

(2) We have $J=I \otimes \iota^{*} j_{*} \mu_{p}$ as subsheaves of $M^{2}$.

We prove this proposition in what follows.

Proof of Proposition 2.5.2 (1) Define the filtration $V^{m}(m \geq 0)$ on $M^{2}$ as the inverse image of $i_{\eta *} i_{\eta}^{*} U^{m} M^{2}$ under the canonical map $M^{2} \rightarrow i_{\eta *} i_{\eta}^{*} M^{2}$. We have $U^{m} M^{2} \subset V^{m} M^{2}$ and $V^{e^{\prime}} M^{2}=J$ by definition, and $V^{0} M^{2}=M^{2}$ by Lemma 2.4.7 for $\eta$. The assertion is reduced to the surjectivity of the natural map

$$
u^{m}: \operatorname{gr}_{U}^{m} M^{2} \longrightarrow \operatorname{gr}_{V}^{m} M^{2}
$$


for any $m$ with $0 \leq m<e^{\prime}$. Indeed, once we show this surjectivity, we will obtain

$$
\begin{aligned}
M^{2}=V^{0} M^{2} & =U^{0} M^{2}+V^{1} M^{2}=U^{0} M^{2}+\left(U^{1} M^{2}+V^{2} M^{2}\right)= \\
\cdots & =U^{0} M^{2}+V^{e^{\prime}} M^{2}=U^{0} M^{2}+J .
\end{aligned}
$$

We prove the surjectivity of (2.5.3). For $m$ with $0 \leq m<e^{\prime}$, consider the following maps:

$$
\operatorname{gr}_{U}^{m} M^{2} \stackrel{u^{m}}{\longrightarrow} \operatorname{gr}_{V}^{m} M^{2} \stackrel{v^{m}}{\longrightarrow} i_{\eta *} i_{\eta}^{*}\left(\operatorname{gr}_{U}^{m} M^{2}\right) \stackrel{\delta^{m}}{\longrightarrow} \bigoplus_{y \in Y^{1}} i_{y *} R^{1} i_{y}^{!}\left(\operatorname{gr}_{U}^{m} M^{2}\right)
$$

where $v^{m}$ denotes the natural injective map obtained from the definition of $V^{\bullet} M^{2}$, and $\delta^{m}$ denotes the boundary map of a localization long exact sequence. By Theorem 2.3.3 (2) and the smoothness of $Y$, the sequence

$$
\operatorname{gr}_{U}^{m} M^{2} \stackrel{v^{m} \circ u^{m}}{\longrightarrow} i_{\eta *} i_{\eta}^{*}\left(\operatorname{gr}_{U}^{m} M^{2}\right) \stackrel{\delta^{m}}{\longrightarrow} \bigoplus_{y \in Y^{1}} i_{y *} R^{1} i_{y}^{!}\left(\operatorname{gr}_{U}^{m} M^{2}\right) .
$$

is exact. To prove the surjectivity of $u_{m}(=(2.5 .3))$, it suffices to check that the composite of $v^{m}$ and $\delta^{m}$ is zero. We show that the stalk of $\delta^{m} \circ v^{m}$ is zero at any point $x \in Y$. Indeed, we are reduced to the case $x \in Y^{1}$ by [Sa1], Lemma 4.2.2, and then we have $\left(U^{m} M^{2}\right)_{\bar{x}}=\left(V^{m} M^{2}\right)_{\bar{x}}$ by Lemma 2.4.7, which implies that $\left(\delta^{m} \circ v^{m}\right)_{\bar{x}}=0$. Thus we obtain Proposition 2.5.2(1).

(2) Put $\mathbb{G}_{\mathrm{m}}=\mathscr{O}_{\mathscr{X}}^{\times}$and

$$
\mathscr{J}:=\operatorname{Ker}\left(R^{3} \iota^{!} \mathbb{G}_{\mathrm{m}} \rightarrow i_{\eta *} i_{\eta}^{*} R^{3} \iota^{!} \mathbb{G}_{\mathrm{m}}\right)
$$

By definition, we have $\mathscr{I} \subset \mathscr{J}$ and $\left({ }_{p} \mathscr{J}\right) \otimes \iota^{*} j_{*} \mu_{p}=J$ (see also (2.3.1)). Hence

$$
I \otimes \iota^{*} j_{*} \mu_{p}=\left({ }_{p} \mathscr{I}\right) \otimes \iota^{*} j_{*} \mu_{p} \subset\left({ }_{p} \mathscr{J}\right) \otimes \iota^{*} j_{*} \mu_{p}=J
$$

as subsheaves of $M^{2}$, and our task is to show the inclusion $\mathscr{I}_{p \text {-tors }} \supset \mathscr{J}_{p \text {-tors }}$. We prove this inclusion in what follows. We put $\mathscr{F}:=R^{3} \iota^{!} \mathbb{G}_{\mathrm{m}}$, and define the coniveau filtration $N^{\bullet} \mathscr{F}$ on $\mathscr{F}$ as follows. We define $N^{0} \mathscr{F}$ to be the full sheaf $\mathscr{F}$. For $\nu \geq 1$, we define

$$
N^{\nu} \mathscr{F}:=\bigcup_{Z \subset Y: \operatorname{codim}_{Y}(Z) \geq \nu} \operatorname{Im}\left(i_{Z *} i_{Z} \mathscr{F} \rightarrow \mathscr{F}\right),
$$

where $Z$ runs through all closed subschemes of $Y$ with $\operatorname{codim}_{Y}(Z) \geq \nu$, and $i_{Z}$ denotes the closed immersion $Z \rightarrow Y$. Since $N^{1} \mathscr{F}=\mathscr{J}$, it suffices to show the following two claims:

Claim. (i) We have $\left(N^{2} \mathscr{F}\right)_{p \text {-tors }}=\left(N^{1} \mathscr{F}\right)_{p \text {-tors }}$.

(ii) We have $N^{2} \mathscr{F} \subset \mathscr{I}$.

Proof of Claim. (i) Because $\mathscr{F}$ is a torsion sheaf, we have

$$
\left(N^{1} \mathscr{F}\right)_{p \text {-tors }} /\left(N^{2} \mathscr{F}\right)_{p \text {-tors }}=\left(\operatorname{gr}_{N}^{1} \mathscr{F}\right)_{p \text {-tors }}
$$

Since $N^{1} \mathscr{F}=\mathscr{J}$, we have

$$
\operatorname{gr}_{N}^{1} \mathscr{F} \subset \bigoplus_{y \in Y^{1}} i_{y *} i_{y}^{!} \mathscr{F}=\bigoplus_{y \in Y^{1}} i_{y *} i_{y}^{*} \mathscr{J}
$$

where for a point $y \in Y, i_{y}$ denotes the canonical map $y \rightarrow Y$. Our task is to show that the stalk $(p \mathscr{J})_{\bar{y}}$ is zero for any $y \in Y^{1}$. We have

$$
{ }_{p} \mathscr{J} \simeq{ }_{p} \mathscr{J} \otimes \iota^{*} j_{*} \mu_{p}=J
$$


by the last equality in (2.5.4) and the assumption that $\zeta_{p} \in k$. On the other hand, $J_{\bar{y}}$ is zero for any $y \in Y^{1}$ by (2.5.1) and Lemma 2.4.7. Hence $(p \mathscr{J})_{\bar{y}}$ is zero for any $y \in Y^{1}$.

(ii) For a closed subscheme $i_{Z}: Z \rightarrow Y$ with $\iota_{Z}: Z \rightarrow Y \rightarrow \mathscr{X}$, there is an exact sequence of torsion sheaves on $Z_{\text {ét }}$

$$
0 \longrightarrow R^{3} \iota_{Z}^{!} \mathbb{G}_{\mathrm{m}} \stackrel{(*)}{\longrightarrow} i_{Z}^{!} R^{3} \iota^{!} \mathbb{G}_{\mathrm{m}}\left(=i_{Z}^{!} \mathscr{F}\right) \longrightarrow\left(R^{3} i_{Z}^{!} \mathbb{Z}_{Y}\right)_{\text {tors }},
$$

which is obtained from the Leray spectral sequence

$$
E_{2}^{u, v}=R^{u} i_{Z}^{!} R^{v} \iota^{!} \mathbb{G}_{\mathrm{m}} \Longrightarrow R^{u+v} \iota_{Z}^{!} \mathbb{G}_{\mathrm{m}}
$$

and the standard purity (2.2.3) (see Lemma 2.2.1 for the injectivity of $(*)$ ). The last sheaf in (2.5.5) is zero if $\operatorname{codim}_{Y}(Z) \geq 2$, because we have a long exact sequence of sheaves

$$
\cdots \longrightarrow R^{2} i_{Z}^{!} \mathbb{Q}_{Y} \longrightarrow R^{2} i_{Z}^{!} \mathbb{Q} / \mathbb{Z}_{Y} \longrightarrow R^{3} i_{Z}^{!} \mathbb{Z}_{Y} \longrightarrow R^{3} i_{Z}^{!} \mathbb{Q}_{Y} \longrightarrow \cdots
$$

and the sheaf $R^{2} i_{Z}^{!} \mathbb{Q} / \mathbb{Z}_{Y}$ is zero by the purity of branch locus (cf. [Gr], II, (3.5.3)). Now we show the inclusion $N^{2} \mathscr{F} \subset \mathscr{I}$. Take a local section $a \in N^{2} \mathscr{F}$. By the definition of $N^{2} \mathscr{F}$, we have $a=i_{Z *}(b)$ for some $i_{Z}: Z \rightarrow Y$ with $\operatorname{codim}_{Y}(Z) \geq 2$ and some local section $b \in i_{Z}^{!} \mathscr{F}$. Since $b$ belongs to $R^{3} \iota_{Z}^{!} \mathbb{G}_{\mathrm{m}}$ by the above facts, $a$ belongs to $\mathscr{I}$ by Lemma 2.2.1. This completes the proof of Claim (ii), Proposition 2.5.2(2) and Theorem 2.3.3.

2.6. Arithmetic filtration. Let $x$ be a point on $Y$, let $A$ be the henselization of the local ring $\mathscr{O}_{\mathscr{X}, x}$ at its maximal ideal, and let $A_{\bar{x}}$ be the strict henselization of $A$ at its maximal ideal. In this subsection, we are concerned with the structure of the group ${ }_{p} \operatorname{Br}(A[1 / p])$. Because $A[1 / p]$ is a UFD containing a primitive $p$-th root of unity $\zeta_{p}$, we have isomorphisms

$$
{ }_{p} \operatorname{Br}(A[1 / p]) \stackrel{\sim}{\sim} H^{2}\left(A[1 / p], \mu_{p}\right) \stackrel{a \mapsto a \cup \zeta_{p}}{\sim} H^{2}\left(A[1 / p], \mu_{p}^{\otimes 2}\right)=: h^{2}\left(A\left[p^{-1}\right]\right) .
$$

We define a descending filtration $U^{\bullet}$ on $h^{2}\left(A\left[p^{-1}\right]\right)$ as follows.

Definition 2.6.1. (1) For $m$ with $0 \leq m \leq e^{\prime}$, we define $U^{m} h^{2}\left(A\left[p^{-1}\right]\right)$ as the inverse image of $\left(U^{m} M^{2}\right)_{\bar{x}}$ under the restriction map

$$
r_{x}: h^{2}\left(A\left[p^{-1}\right]\right) \longrightarrow H^{2}\left(A_{\bar{x}}\left[p^{-1}\right], \mu_{p}^{\otimes 2}\right)=\left(M^{2}\right)_{\bar{x}} .
$$

(2) For $m \geq e^{\prime}+1$, we define $U^{m} h^{2}\left(A\left[p^{-1}\right]\right):=0$.

By definition, we have $\operatorname{gr}_{U}^{e^{\prime}} h^{2}\left(A\left[p^{-1}\right]\right)=\operatorname{Ker}\left(r_{x}\right)$. The following result will play key roles in the next section:

Theorem 2.6.2. For $0 \leq m \leq e^{\prime}$ we have isomorphisms

$$
\operatorname{gr}_{U}^{m} h^{2}\left(A\left[p^{-1}\right]\right) \simeq \begin{cases}\Omega_{R, \log }^{2} \oplus \Omega_{R, \log }^{1} & (m=0), \\ \Omega_{R}^{1} & \left(0<m<e^{\prime}, p \nmid m\right), \\ \Omega_{R}^{1} / \mathscr{Z}_{R}^{1} \oplus R / R^{p} & \left(0<m<e^{\prime}, p \mid m\right), \\ \left.{ }_{p} \operatorname{Br}(x) \oplus H^{1}(x, \mathbb{Z} / p \mathbb{Z})\right) \otimes \mu_{p}(k) & \left(m=e^{\prime}\right),\end{cases}
$$

where we put $R:=A /(T)$ (see $\$ 2.1$ for $T$ ) and wrote $\Omega_{R}^{a}$, $\mathscr{Z}_{R}^{a}$ and $\Omega_{R, \log }^{a}$ for

$$
\Gamma\left(\operatorname{Spec}(R),\left.\Omega_{Y}^{a}\right|_{\operatorname{Spec}(R)}\right), \quad \Gamma\left(\operatorname{Spec}(R),\left.\mathscr{Z}_{Y}^{a}\right|_{\operatorname{Spec}(R)}\right) \quad \text { and } \quad \Gamma\left(\operatorname{Spec}(R),\left.\Omega_{Y, \log }^{a}\right|_{\operatorname{Spec}(R)}\right),
$$


respectively. Further if $x$ is a closed point on $Y$, then we have a canonical direct decomposition

$$
h^{2}\left(A\left[p^{-1}\right]\right) \simeq U^{0} h^{2}\left(A\left[p^{-1}\right]\right) \oplus\left(\left(I_{\bar{x}}\right)^{G_{x}} \otimes \mu_{p}(k)\right),
$$

where $G_{x}$ denotes the absolute Galois group of $\kappa(x)$, and I denotes the sheaf on $Y_{\text {ét }}$ defined in $\$ 2.3$

We first show the following lemma, where we do not assume that $x$ is a closed point on $Y$ :

Lemma 2.6.4. (1) There is a short exact sequence

$$
0 \longrightarrow{ }_{p} \operatorname{Br}(x) \oplus H^{1}(x, \mathbb{Z} / p \mathbb{Z}) \longrightarrow H^{2}\left(A\left[p^{-1}\right], \mu_{p}\right) \longrightarrow H^{2}\left(A_{\bar{x}}\left[p^{-1}\right], \mu_{p}\right)^{G_{x}} \longrightarrow 0 .
$$

(2) Let $A_{\eta_{x}}$ (resp. $A_{\eta_{\bar{x}}}$ ) be the henselization of $A$ (resp. $\left.A_{\bar{x}}\right)$ at the prime ideal TA (resp. $\left.T A_{\bar{x}}\right)$. Let $\eta_{x}\left(\right.$ resp. $\left.\eta_{\bar{x}}\right)$ be the closed point of $\operatorname{Spec}\left(A_{\eta_{x}}\right)\left(\operatorname{resp} . \operatorname{Spec}\left(A_{\eta_{\bar{x}}}\right)\right)$. Then there is a short exact sequence

$$
0 \longrightarrow H^{1}(x, \mathbb{Q} / \mathbb{Z}) \longrightarrow H_{\eta_{x}}^{3}\left(A_{\eta_{x}}, \mathbb{G}_{\mathrm{m}}\right) \longrightarrow H_{\eta_{\bar{x}}}^{3}\left(A_{\eta_{\bar{x}}}, \mathbb{G}_{\mathrm{m}}\right)^{G_{x}} \longrightarrow 0 .
$$

Proof of Lemma 2.6.4 (1) Consider the short exact sequence

$$
0 \longrightarrow H_{\text {Gal }}^{1}\left(G_{x},\left(A_{\bar{x}}\left[p^{-1}\right]\right)^{\times} / p\right) \longrightarrow H^{2}\left(A\left[p^{-1}\right], \mu_{p}\right) \longrightarrow H^{2}\left(A_{\bar{x}}\left[p^{-1}\right], \mu_{p}\right)^{G_{x}} \longrightarrow 0
$$

obtained from the Hochschild-Serre spectral sequence

$$
E_{2}^{u, v}=H_{\text {Gal }}^{u}\left(G_{x}, H^{v}\left(A_{\bar{x}}\left[p^{-1}\right], \mu_{p}\right)\right) \Longrightarrow H^{u+v}\left(A\left[p^{-1}\right], \mu_{p}\right),
$$

and the fact that $\operatorname{cd}_{p}\left(G_{x}\right)=1$. We calculate the first term in this short exact sequence. Let $U_{A_{\bar{x}}\left[p^{-1}\right]}^{1}$ be the subgroup $\left(1+T \cdot A_{\bar{x}}\right)$ of $\left(A_{\bar{x}}\left[p^{-1}\right]\right)^{\times}$and let $R_{\bar{x}}$ be the strict henselization of $R$ at its maximal ideal. By an easier variant of Lemma 2.4.1, we have the following short exact sequence of discrete $G_{x}$-modules:

$$
0 \longrightarrow U_{A_{\bar{x}}\left[p^{-1}\right]}^{1} / p \longrightarrow\left(A_{\bar{x}}\left[p^{-1}\right]\right)^{\times} / p \longrightarrow \Omega_{R_{\bar{x}}, \log }^{1} \oplus \mathbb{Z} / p \mathbb{Z} \longrightarrow 0,
$$

and $U_{A_{\bar{x}}\left[p^{-1}\right]}^{1} / p$ is a finitely successive extension of additive $G_{x}$-modules. Hence we have

$$
H_{\text {Gal }}^{1}\left(G_{x},\left(A_{\bar{x}}\left[p^{-1}\right]\right)^{\times} / p\right) \simeq H_{\text {Gal }}^{1}\left(G_{x}, \Omega_{R_{\bar{x}}, \log }^{1}\right) \oplus H_{\text {Gal }}^{1}\left(G_{x}, \mathbb{Z} / p \mathbb{Z}\right) .
$$

The assertion follows from the following fact ([G], I, Corollaire 6.2, II, Corollaire 2.5):

$$
H_{\text {Gal }}^{1}\left(G_{x}, \Omega_{R_{\bar{x}}, \log }^{1}\right) \simeq H^{1}\left(R,\left.\Omega_{Y, \log }^{1}\right|_{\operatorname{Spec}(R)}\right) \simeq{ }_{p} \operatorname{Br}(R) \simeq{ }_{p} \operatorname{Br}(x) .
$$

(2) One can check the assertion by calculating the Hochschild-Serre spectral sequence

$$
E_{2}^{u, v}=H_{\text {Gal }}^{u}\left(G_{x}, H_{\eta_{\bar{x}}}^{v}\left(A_{\eta_{\bar{x}}}, \mathbb{G}_{\mathrm{m}}\right)\right) \Longrightarrow H_{\eta_{x}}^{u+v}\left(A_{\eta_{x}}, \mathbb{G}_{\mathrm{m}}\right),
$$

using the standard purity (2.2.3). The details are straight-forward and left to the reader.

Proof of Theorem 2.6.2. The isomorphism (2.6.3) for $m=e^{\prime}$ follows from Lemma 2.6.4(1). The isomorphism (2.6.3) for $0 \leq m<e^{\prime}$ follows from Theorem 2.3.3 (2) and the fact that $\left(U^{1} M^{2}\right)_{\bar{x}}$ is a finitely successive extension of additive $G_{x}$-modules. Next we prove the direct decomposition. Assume that $x$ is a closed point on $Y$. Then since $\kappa(x)$ is finite, we have $\operatorname{Br}(x)=0$ and a commutative diagram with exact rows by Lemma 2.6.4

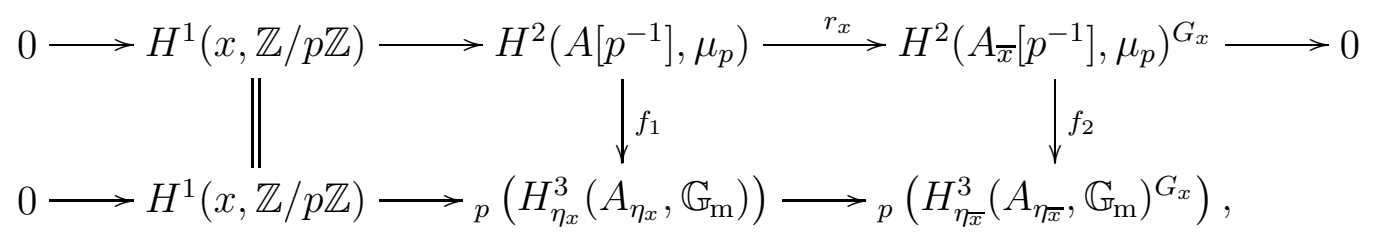


where $f_{1}$ and $f_{2}$ are the following composite maps, respectively (cf. (2.3.1)):

$$
\begin{aligned}
& f_{1}: H^{2}\left(A\left[p^{-1}\right], \mu_{p}\right)={ }_{p} H^{2}\left(A\left[p^{-1}\right], \mathbb{G}_{\mathrm{m}}\right) \longrightarrow{ }_{p} H_{\eta_{x}}^{3}\left(A_{\eta_{x}}, \mathbb{G}_{\mathrm{m}}\right), \\
& f_{2}: H^{2}\left(A_{\bar{x}}\left[p^{-1}\right], \mu_{p}\right)={ }_{p} H^{2}\left(A_{\bar{x}}\left[p^{-1}\right], \mathbb{G}_{\mathrm{m}}\right) \longrightarrow{ }_{p} H_{\eta_{\bar{x}}}^{3}\left(A_{\eta_{\bar{x}}}, \mathbb{G}_{\mathrm{m}}\right) .
\end{aligned}
$$

Note that $\operatorname{Ker}\left(f_{2}\right) \simeq\left(I_{\bar{x}}\right)^{G_{x}}$ by the definition of $I$ and the fact that $\operatorname{Br}\left(A_{\bar{x}}\right) \simeq \operatorname{Br}(\bar{x})=0$. Hence applying the snake lemma to (2.6.5), we obtain $\operatorname{Ker}\left(f_{1}\right) \simeq\left(I_{\bar{x}}\right)^{G_{x}}$ and a canonical injective map

$$
\left(I_{\bar{x}}\right)^{G_{x}} \otimes \mu_{p}(k) \hookrightarrow h^{2}\left(A\left[p^{-1}\right]\right) .
$$

Finally by Theorem 2.3.3 (1) and a diagram chase on the diagram (2.6.5) tensored with $\mu_{p}(k)$, one can easily check that

$$
\begin{aligned}
& U^{0} h^{2}\left(A\left[p^{-1}\right]\right)+\left(\left(I_{\bar{x}}\right)^{G_{x}} \otimes \mu_{p}(k)\right)=h^{2}\left(A\left[p^{-1}\right]\right), \\
& U^{0} h^{2}\left(A\left[p^{-1}\right]\right) \cap\left(\left(I_{\bar{x}}\right)^{G_{x}} \otimes \mu_{p}(k)\right)=0 .
\end{aligned}
$$

This completes the proof.

Corollary 2.6.6. Let $A_{\eta_{x}}$ be as in Lemma 2.6.4 and assume that $x$ is a closed point on $Y$. Put $K_{\eta_{x}}:=\operatorname{Frac}\left(A_{\eta_{x}}\right)=A_{\eta_{x}}\left[p^{-1}\right]$. Then the following natural map is injective:

$$
U^{0} h^{2}\left(A\left[p^{-1}\right]\right) \rightarrow h^{2}\left(K_{\eta_{x}}\right):=H^{2}\left(K_{\eta_{x}}, \mu_{p}^{\otimes 2}\right) .
$$

Proof. Define the filtration $U^{m}\left(0 \leq m \leq e^{\prime}\right)$ on $h^{2}\left(K_{\eta_{x}}\right)$ as the inverse image of $\left(U^{m} M^{2}\right)_{\bar{\eta}}$ under the natural map $h^{2}\left(K_{\eta_{x}}\right) \rightarrow\left(M^{2}\right)_{\bar{\eta}}$, where $\eta$ denotes the generic point of $Y$. For $0 \leq m<e^{\prime}$, the induced map $\operatorname{gr}_{U}^{m} h^{2}\left(A\left[p^{-1}\right]\right) \rightarrow \operatorname{gr}_{U}^{m} h^{2}\left(K_{\eta_{x}}\right)$ is injective by Theorem 2.3 .3 (2). The map $U^{e^{\prime}} h^{2}\left(A\left[p^{-1}\right]\right) \rightarrow U^{e^{\prime}} h^{2}\left(K_{\eta_{x}}\right)$ is injective by (2.6.3) for $m=e^{\prime}$ and Lemma2.6.4(2).

Remark 2.6.7. We mention here the relation between the isomorphisms in (2.6.3) and symbols in $K_{2}^{M}\left(A\left[p^{-1}\right]\right)$. We define

$$
U^{0} K_{2}^{M}\left(A\left[p^{-1}\right]\right):=K_{2}^{M}\left(A\left[p^{-1}\right]\right),
$$

and define $U^{m} K_{2}^{M}\left(A\left[p^{-1}\right]\right)(m \geq 1)$ as the subgroup generated by symbols of the form

$$
\left\{1+f \cdot T^{m}, g\right\} \quad \text { with } f \in A \text { and } g \in\left(A\left[p^{-1}\right]\right)^{\times} .
$$

We define the filtration $U_{\mathrm{sym}}^{m}(m \geq 0)$ on $h^{2}\left(A\left[p^{-1}\right]\right)$ as the image of $U^{m} K_{2}^{M}\left(A\left[p^{-1}\right]\right)$ under the symbol map $K_{2}^{M}\left(A\left[p^{-1}\right]\right) \rightarrow h^{2}\left(A\left[p^{-1}\right]\right)$. Then we have

$$
U_{\text {sym }}^{m} h^{2}\left(A\left[p^{-1}\right]\right) \subset U^{m} h^{2}\left(A\left[p^{-1}\right]\right) \quad\left(0 \leq m \leq e^{\prime}\right)
$$

by definition. By the same arguments as for Lemma 2.4.1, we obtain

$$
\begin{aligned}
& U_{\mathrm{sym}}^{e^{\prime}+1} h^{2}\left(A\left[p^{-1}\right]\right)=U^{e^{\prime}+1} h^{2}\left(A\left[p^{-1}\right]\right)=0 \\
& \operatorname{gr}_{U_{\mathrm{sym}}^{m}}^{m} h^{2}\left(A\left[p^{-1}\right]\right) \simeq \operatorname{gr}_{U}^{m} h^{2}\left(A\left[p^{-1}\right]\right) \stackrel{(2.6 .3)}{\sim} \begin{cases}\Omega_{R}^{1} & \left(0<m<e^{\prime}, p \nmid m\right) \\
\Omega_{R}^{1} / Z_{R}^{1} \oplus R / R^{p} & \left(0<m<e^{\prime}, p \mid m\right) .\end{cases}
\end{aligned}
$$

By more detailed calculations, one can show that

$$
\operatorname{gr}_{U_{\text {sym }}}^{m} h^{2}\left(A\left[p^{-1}\right]\right) \simeq \operatorname{gr}_{U}^{m} h^{2}\left(A\left[p^{-1}\right]\right)
$$

for $m=0, e^{\prime}$ as well (hence $U_{\mathrm{sym}}^{m} h^{2}\left(A\left[p^{-1}\right]\right)=U^{m} h^{2}\left(A\left[p^{-1}\right]\right)$ for any $m \geq 0$ ), but we will not use this fact in this paper. 


\section{KEY THEOREM FOR BRAUER GROUPS OF LOCAL RINGS}

In this section, we are concerned with a local ring $A$ satisfying the following condition:

(\$) $A$ is obtained by the henselization of a regular scheme $\mathscr{X}$ satisfying the conditions in $\$ 2.1$ at a closed point of characteristic $p$.

Note that $A\left[p^{-1}\right]$ contains primitive $p$-th roots of unity. $\operatorname{Put} \operatorname{br}\left(A\left[p^{-1}\right]\right):={ }_{p} \operatorname{Br}\left(A\left[p^{-1}\right]\right)$, and fix a primitive $p$-th root of unity $\zeta_{p} \in A\left[p^{-1}\right]$.

3.1. Let $\mathrm{br}^{\mathrm{a}}\left(A\left[p^{-1}\right]\right)$ be the image of $U^{0} h^{2}\left(A\left[p^{-1}\right]\right)$ (cf. $\left.\$ 2.6\right)$ under the composite of isomorphisms

$$
h^{2}\left(A\left[p^{-1}\right]\right)=H^{2}\left(A\left[p^{-1}\right], \mu_{p}^{\otimes 2}\right) \stackrel{x \otimes \zeta_{p} \mapsto x}{\sim} H^{2}\left(A\left[p^{-1}\right], \mu_{p}\right) \stackrel{\sim}{\longrightarrow} \operatorname{br}\left(A\left[p^{-1}\right]\right) .
$$

Note that $\operatorname{br}^{\mathrm{a}}\left(A\left[p^{-1}\right]\right)$ does not depend on the choice of $\zeta_{p}$. The aim of this section is to prove the following key result.

Theorem 3.1.1. Let $A$ be as above, and let $Q$ be the set of all closed points on $\operatorname{Spec}\left(A\left[p^{-1}\right]\right)$. Then the specialization map

$$
\operatorname{br}^{\mathrm{a}}\left(A\left[p^{-1}\right]\right) \longrightarrow \prod_{v \in Q} \operatorname{br}(v)
$$

is injective.

We will prove Theorem 3.1.1 in $\S \S 3.2+3.4$ below. The following direct consequence of Theorem 3.1.1 and Theorem 2.6.2 will be used in the proof of Theorem 1.3.1 (see $\$ 7$ below).

Corollary 3.1.2. Let $A$ and $Q$ be as above, and assume that the purity of Brauer groups holds for $\operatorname{Spec}(A)$. Then the specialization map

$$
\operatorname{Br}\left(A\left[p^{-1}\right]\right)_{p \text {-tors }} \longrightarrow \prod_{v \in Q} \operatorname{Br}(v)_{p \text {-tors }}
$$

is injective.

The prime-to- $p$ variant of Corollary 3.1.2 will be mentioned at the end of this section (see Remark 3.4.2 below). Before starting the proof of Theorem 3.1.1, we explain an outline of the proof. We will prove Theorem 3.1.1 by induction on $\operatorname{dim}(A) \geq 2$. More precisely, for a given $A$ satisfying $(\boldsymbol{A})$ with $\operatorname{dim}(A) \geq 3$, we will construct a subset $P$ of prime ideals of $A$ of height 1 for which the residue ring $B_{\mathfrak{p}}:=A / \mathfrak{p}$ for any $\mathfrak{p} \in P$ again satisfies $(\boldsymbol{\uparrow})$ and for which the specialization map

$$
\operatorname{br}^{\mathrm{a}}\left(A\left[p^{-1}\right]\right) \longrightarrow \prod_{\mathfrak{p} \in P} \operatorname{br}^{\mathrm{a}}\left(B_{\mathfrak{p}}\left[p^{-1}\right]\right)
$$

is injective (note that the subgroup $\mathrm{br}^{\mathrm{a}} \subset$ br is functorial by definition). By the transitivity of specialization maps, this argument reduces Theorem 3.1 .1 to the case $\operatorname{dim}(A)=2$. We construct such a set of prime ideals assuming that $\operatorname{dim}(A)>3$ in $\$ 3.2$ (see Proposition 3.2.3 below). The case $\operatorname{dim}(A)=3$ is more delicate and will be treated in $\S 3.3$ (see Corollary 3.3 .2 below). Finally in $\$ 3.4$ we will prove the 2-dimensional case using results in [S1]. 
3.2. Reduction to the 3-dimensional case. We reduce Theorem 3.1.1 with $\operatorname{dim}(A)>3$ to the 3-dimensional case. Since $\operatorname{br}^{\mathrm{a}}\left(A\left[p^{-1}\right]\right)$ is isomorphic to $U^{0} h^{2}\left(A\left[p^{-1}\right]\right)$ by definition, we investigate $U^{0} h^{2}\left(A\left[p^{-1}\right]\right)$. Fix a prime element $T \in A$ which generates the radical of $(p) \subset A$. We define the arithmetic filtration $U^{i}(i \geq 1)$ on $U^{0} h^{2}\left(A\left[p^{-1}\right]\right)$ using this $T$ (see $\$ 2.6)$. Let $R$ be the residue ring $A /(T)$, and let $e$ be the absolute ramification index of $A$, i.e., the order of $p$ with respect to $T$. Put $e^{\prime}:=p e /(p-1)$. We have $U^{e^{\prime}+1} h^{2}\left(A\left[p^{-1}\right]\right)=0$ and the following isomorphisms for $0 \leq i \leq e^{\prime}$ by Theorem 2.6.2 (see also Remark 2.6.7):

$$
\operatorname{gr}_{U}^{i} h^{2}\left(A\left[p^{-1}\right]\right) \simeq \begin{cases}\Omega_{R, \log }^{2} \oplus \Omega_{R, \log }^{1} & (i=0), \\ \Omega_{R}^{1} & \left(0<i<e^{\prime},(p, i)=1\right), \\ \Omega_{R}^{1} / \mathscr{Z}_{R}^{1} \oplus R / R^{p} & \left(0<i<e^{\prime}, p \mid i\right), \\ H^{1}\left(\mathbb{F}_{q}, \mathbb{Z} / p \mathbb{Z}\right) \otimes \mu_{p}(k) & \left(i=e^{\prime}\right),\end{cases}
$$

where $\mathbb{F}_{q}$ denotes the residue field of $A$. Now put $r:=\operatorname{dim}(A)-1$, and fix prime elements $X_{1}, X_{2}, \ldots, X_{r} \in A$ such that $\left(T, X_{1}, X_{2}, \ldots, X_{r}\right)$ is a system of regular parameters of $A$. Let $P_{0} \subset A$ be the subset of polynomials in $X_{1}, X_{2}, \ldots, X_{r}$ with $\mathbb{Z}$-coefficients whose constant term is zero and whose linear part is non-zero. For each $f \in P_{0}$, the residue ring $A /(f)$ is an integral $r$-dimensional henselian regular local ring with residue field $\mathbb{F}_{q}$ satisfying $(\boldsymbol{\beta})$ (its regularity can be checked by counting the dimension of its cotangent space), and $T+(f)$ generates the radical of $(p) \subset A /(f)$. In particular, an arbitrary contained in $P_{0}$ is a prime element of $A$. Now we define the equivalence relation $\sim$ on $P_{0}$ by the law that $f_{1} \sim f_{2}$ iff $f_{1}=u \cdot f_{2}$ for some $u \in A^{\times}$. Put $P:=P_{0} / \sim$, which corresponds to a subset of prime ideals of $A$ of height 1 . For a prime ideal $\mathfrak{p} \in P$, put

$$
B_{\mathfrak{p}}:=A / \mathfrak{p}, \quad T_{\mathfrak{p}}:=T+\mathfrak{p} \in B_{\mathfrak{p}} \quad \text { and } \quad C_{\mathfrak{p}}:=B_{\mathfrak{p}} /\left(T_{\mathfrak{p}}\right)=R / \mathfrak{p} R .
$$

We define the arithmetic filtration $U^{\bullet}$ on $h^{2}\left(B_{\mathfrak{p}}\left[p^{-1}\right]\right)$ using the prime element $T_{\mathfrak{p}} \in B_{\mathfrak{p}}$ (see $\$ 2.6)$. By definition, the specialization map $h^{2}\left(A\left[p^{-1}\right]\right) \rightarrow h^{2}\left(B_{\mathfrak{p}}\left[p^{-1}\right]\right)$ preserves $U^{\bullet}$ and induces the following map for each $i$ with $0 \leq i \leq e^{\prime}$ :

$$
\operatorname{gr}_{U}^{i} h^{2}\left(A\left[p^{-1}\right]\right) \longrightarrow \operatorname{gr}_{U}^{i} h^{2}\left(B_{\mathfrak{p}}\left[p^{-1}\right]\right)
$$

We first note the following standard fact:

Lemma 3.2.2. Suppose that $\operatorname{dim}(A) \geq 3$, and let a be an integer with $0 \leq a \leq \operatorname{dim}(A)-2$. Then the specialization map of differential modules

$$
\Omega_{R}^{a} \longrightarrow \prod_{\mathfrak{p} \in P} \Omega_{C_{\mathfrak{p}}}^{a}
$$

is injective.

Proof. This is directly shown by taking $\mathfrak{p}=\left(X_{j}-X_{j^{\prime}}^{m}\right)$ with $j \neq j^{\prime}$ and $m=1,2, \ldots$

Proposition 3.2.3. Suppose that $\operatorname{dim}(A)>3$. Then for any $i$ with $0 \leq i \leq e^{\prime}$ the specialization map

$$
\theta_{i}: \operatorname{gr}_{U}^{i} h^{2}\left(A\left[p^{-1}\right]\right) \longrightarrow \prod_{\mathfrak{p} \in P} \operatorname{gr}_{U}^{i} h^{2}\left(B_{\mathfrak{p}}\left[p^{-1}\right]\right)
$$

is injective. Consequently, the specialization map

$$
\operatorname{br}^{\mathrm{a}}\left(A\left[p^{-1}\right]\right) \longrightarrow \prod_{\mathfrak{p} \in P} \operatorname{br}^{\mathrm{a}}\left(B_{\mathfrak{p}}\left[p^{-1}\right]\right)
$$


is injective.

Proof. By (3.2.1) for $i=e^{\prime}, \theta_{e^{\prime}}$ is injective, because the residue fields of $A$ and $B_{\mathfrak{p}}$ are the same. Next suppose that $i<e^{\prime}$. Then the groups on the both hand sides of the map $\theta_{i}$ are described by differential modules of $R$ and $C_{\mathfrak{p}}$, respectively, by (3.2.1). Because $\theta_{i}$ is compatible with the specialization of differential forms, it is injective by Lemma 3.2.2 and the fact that $\Omega_{R}^{q} / \mathscr{Z}_{R}^{q}$ is a subgroup of $\Omega_{R}^{q+1}$. This completes the proof.

Thus Theorem 3.1.1 with $\operatorname{dim}(A)>3$ is reduced to the 3 -dimensional case.

3.3. Reduction to the 2-dimensional case. We reduce Theorem 3.1.1 to the 2-dimensional case. Let the notation be as in the previous subsection, and suppose that $\operatorname{dim}(A)=3$. Lemma 3.2.2 together with (3.2.1) implies that the map $\theta_{i}$ in Proposition 3.2.3 is injective if $i=e^{\prime}$ or if $0<i<e^{\prime}$ with $p \nmid i$. If $i=0$, then the restriction of $\theta_{0}$ to $\Omega_{R, \log }^{1}$ is still injective:

$$
\Omega_{R, \log }^{1} \longleftrightarrow \operatorname{gr}_{U}^{0} h^{2}\left(A\left[p^{-1}\right]\right) \stackrel{\theta_{0}}{\longrightarrow} \prod_{\mathfrak{p} \in P} \operatorname{gr}_{U}^{0} h^{2}\left(B_{\mathfrak{p}}\left[p^{-1}\right]\right) \simeq \prod_{\mathfrak{p} \in P} \Omega_{C_{\mathfrak{p}}, \log }^{1} .
$$

If $0<i<e^{\prime}$ and $p \mid i$, then the restriction of $\theta_{i}$ to $R / R^{p}$ is also injective:

$$
R / R^{p} \longleftrightarrow \operatorname{gr}_{U}^{i} h^{2}\left(A\left[p^{-1}\right]\right) \stackrel{\theta_{i}}{\longrightarrow} \prod_{\mathfrak{p} \in P} \operatorname{gr}_{U}^{i} h^{2}\left(B_{\mathfrak{p}}\left[p^{-1}\right]\right) \simeq \prod_{\mathfrak{p} \in P} C_{\mathfrak{p}} /\left(C_{\mathfrak{p}}\right)^{p} .
$$

Thus it remains to deal with the following subgroups in case $\operatorname{dim}(A)=3$ :

$$
\begin{aligned}
\Omega_{R, \log }^{2} \subset \operatorname{gr}_{U}^{0} h^{2}\left(A\left[p^{-1}\right]\right) & \text { and } \\
\Omega_{R}^{1} / \mathscr{Z}_{R}^{1} \subset \operatorname{gr}_{U}^{i} h^{2}\left(A\left[p^{-1}\right]\right) & \text { with } 0<i<e^{\prime} \text { and } p \mid i .
\end{aligned}
$$

These subgroups are treated by a subset of prime ideals of height 1 which is different from $P$. Let $X_{1}, X_{2}, T \in A$ and $e$ be as in $\oint 3.2$ (note that $r=2$ ). For integer $m>0$, put

$$
B_{m}:=A /\left(T-X_{1}^{m}\right) \text {. }
$$

It is easy to see that $B_{m}$ is a 2-dimensional henselian regular local ring satisfying $(\boldsymbol{A})$, and that $x_{m}:=X_{1}+\left(T-X_{1}^{m}\right) \in B_{m}$ generates the radical of $(p) \subset B_{m}$ (hence the absolute ramification index of $B_{m}$ is $m \cdot e$ ). In particular, $T-X_{1}^{m}$ is a prime element of $A$ which does not belong to $P$. Now let us define the arithmetic filtration $U^{\bullet}$ on $h^{2}\left(B_{m}\left[p^{-1}\right]\right)$ using the prime $x_{m} \in B_{m}$. Since $T=\left(x_{m}\right)^{m}$ in $B_{m}$, the specialization map $h^{2}\left(A\left[p^{-1}\right]\right) \rightarrow h^{2}\left(B_{m}\left[p^{-1}\right]\right)$ induces the following maps:

$$
\begin{aligned}
U^{i} h^{2}\left(A\left[p^{-1}\right]\right) & \longrightarrow U^{m i} h^{2}\left(B_{m}\left[p^{-1}\right]\right), \\
\operatorname{gr}_{U}^{i} h^{2}\left(A\left[p^{-1}\right]\right) & \longrightarrow U^{m i} / U^{m(i+1)} h^{2}\left(B_{m}\left[p^{-1}\right]\right),
\end{aligned}
$$

where for integers $b>a \geq 0, U^{a} / U^{b} h^{2}\left(B_{m}\left[p^{-1}\right]\right)$ denotes $U^{a} h^{2}\left(B_{m}\left[p^{-1}\right]\right) / U^{b} h^{2}\left(B_{m}\left[p^{-1}\right]\right)$. We investigate the following composite map for integers $i, m>0$ with $0<i<e^{\prime}$ and $p \mid i$ :

$$
\pi_{i, m}: \Omega_{R}^{1} / \mathscr{Z}_{R}^{1} \longleftrightarrow \operatorname{gr}_{U}^{i} h^{2}\left(A\left[p^{-1}\right]\right) \longrightarrow U^{m i} / U^{m(i+1)} h^{2}\left(B_{m}\left[p^{-1}\right]\right)
$$

and the following composite map for $m>0$ :

$$
\tau_{0, m}: \Omega_{R, \log }^{2} \longleftrightarrow \operatorname{gr}_{U}^{0} h^{2}\left(A\left[p^{-1}\right]\right) \longrightarrow U^{0} / U^{m} h^{2}\left(B_{m}\left[p^{-1}\right]\right) .
$$

The following proposition will reduce Theorem 3.1.1 to the 2-dimensional case: 
Proposition 3.3.1. $\quad$ (1) Let $i$ be an integer with $0<i<e^{\prime}$ and $p \mid i$. Then the map

$$
\pi_{i}: \Omega_{R}^{1} / \mathscr{Z}_{R}^{1} \longrightarrow \prod_{m=2}^{\infty} U^{m i} / U^{m(i+1)} h^{2}\left(B_{m}\left[p^{-1}\right]\right),
$$

defined as the product of $\left\{\pi_{i, m}\right\}_{m \geq 2}$, is injective.

(2) The map

$$
\tau_{0}: \Omega_{R, \log }^{2} \longrightarrow \prod_{m=2}^{\infty} U^{0} / U^{m} h^{2}\left(B_{m}\left[p^{-1}\right]\right)
$$

defined as the product of $\left\{\tau_{0, m}\right\}_{m \geq 2}$, is injective.

Corollary 3.3.2. The specialization map

$$
\operatorname{br}^{\mathrm{a}}\left(A\left[p^{-1}\right]\right) \longrightarrow \prod_{\mathfrak{p} \in P} \operatorname{br}^{\mathrm{a}}\left(B_{\mathfrak{p}}\left[p^{-1}\right]\right) \times \prod_{m=2}^{\infty} \operatorname{br}^{\mathrm{a}}\left(B_{m}\left[p^{-1}\right]\right)
$$

is injective.

We prove Proposition 3.3.1 in what follows. We define the elements $x, y \in R$ as

$$
x:=X_{1}+(T) \quad \text { and } \quad y:=X_{2}+(T) .
$$

Put

$$
C:=R /(x) \quad \text { and } \quad z:=y+(x) \in C .
$$

For $m>0$, put

$$
x_{m}:=X_{1}+\left(T-X_{1}^{m}\right) \in B_{m} .
$$

Note that $B_{m} /\left(x_{m}\right) \simeq C$ for any $m>0$ and that $z$ is a prime element of $C$. We start the proof of Proposition 3.3.1(1). Fix a positive integer $i$ with $0<i<e^{\prime}$ and $p \mid i$. For integer $\nu \geq 0$, put

$$
\mathrm{F}^{\nu} \Omega_{R}^{1}:=x^{\nu} \cdot \Omega_{R}^{1} \quad \text { and } \quad \mathrm{F}^{\nu}\left(\Omega_{R}^{1} / \mathscr{Z}_{R}^{1}\right):=\left(\mathrm{F}^{\nu} \Omega_{R}^{1}+\mathscr{Z}_{R}^{1}\right) / \mathscr{Z}_{R}^{1} \subset \Omega_{R}^{1} / \mathscr{Z}_{R}^{1} .
$$

These filtrations are not finite, but exhaustive, i.e.,

$$
\bigcap_{\nu \geq 0} \mathrm{~F}^{\nu} \Omega_{R}^{1}=0=\bigcap_{\nu \geq 0} \mathrm{~F}^{\nu}\left(\Omega_{R}^{1} / \mathscr{Z}_{R}^{1}\right),
$$

because $\mathrm{F}^{\nu}\left(\Omega_{R}^{1} / \mathscr{Z}_{R}^{1}\right) \subset x^{\nu-1} \cdot \Omega_{R}^{2}$. Hence it is enough to show that

$$
\operatorname{Ker}\left(\prod_{m=2}^{\nu+2} \pi_{i, m}\right) \subset \mathrm{F}^{\nu+1}\left(\Omega_{R}^{1} / \mathscr{Z}_{R}^{1}\right) \quad \text { for } \quad \nu \geq 0 .
$$

Here $\prod_{m=2}^{\nu+2} \pi_{i, m}$ means the map

$$
\Omega_{R}^{1} / \mathscr{Z}_{R}^{1} \longrightarrow \prod_{m=2}^{\nu+2} U^{m i} / U^{m(i+1)} h^{2}\left(B_{m}\left[p^{-1}\right]\right)
$$

induced by $\pi_{i, m}$ with $m=2,3, \ldots, \nu+2$. We compute the filtration $\mathrm{F}^{\bullet}\left(\Omega_{R}^{1} / \mathscr{Z}_{R}^{1}\right)$ in Lemma 3.3.6 below and then prove this inclusion in Lemma 3.3.8 below. For $\nu \geq 0$, we have the following exact sequence of $C$-modules:

$$
0 \longrightarrow C \stackrel{\alpha_{\nu}}{\longrightarrow} \operatorname{gr}_{\mathrm{F}}^{\nu} \Omega_{R}^{1} \stackrel{\beta_{\nu}}{\longrightarrow} \Omega_{C}^{1} \longrightarrow 0,
$$


where $\alpha_{\nu}$ and $\beta_{\nu}$ are defined as follows:

$$
\begin{aligned}
\alpha_{\nu}(\bar{a}) & :=a x^{\nu} d x+\mathrm{F}^{\nu+1} \Omega_{R}^{1} & & (a \in R, \bar{a}=a+(x) \in C) \\
\beta_{\nu}\left(x^{\nu}(a d x+b d y)\right) & :=\bar{b} d z & & (b \in R, \bar{b}=b+(x) \in C) .
\end{aligned}
$$

Let

$$
\gamma_{\nu}: C \longrightarrow \operatorname{gr}_{\mathrm{F}}^{\nu}\left(\Omega_{R}^{1} / \mathscr{Z}_{R}^{1}\right), \quad \bar{a} \mapsto a x^{\nu} d x+\left(\mathrm{F}^{\nu+1} \Omega_{R}^{1}+\mathscr{Z}_{R}^{1}\right)
$$

be the map induced by $\alpha_{\nu}$. We prove the following lemma:

Lemma 3.3.6. For $\nu \geq 0$, the following holds:

(1) $\gamma_{\nu}$ annihilates $C^{p}$.

(2) If $p \nmid(\nu+1)$, then $\gamma_{\nu}$ is zero.

(3) If $p \mid \nu$, then $\operatorname{gr}_{\mathrm{F}}^{\nu}\left(\Omega_{R}^{1} / \mathscr{Z}_{R}^{1}\right)=0$.

Proof. (1) follows from the fact that $d\left(a^{p} x^{\nu} d x\right)=0$ in $\Omega_{R}^{2}$.

(2) If $\nu+1$ is prime to $p$, then we have

$$
a x^{\nu} d x=-\frac{x^{\nu+1}}{\nu+1} d a+d\left(\frac{a x^{\nu+1}}{\nu+1}\right) \quad(a \in R)
$$

in $\Omega_{R}^{1}$. Hence $a x^{\nu} d x \in \mathrm{F}^{\nu} \Omega_{R}^{1}$ is contained in $\mathrm{F}^{\nu+1}\left(\Omega_{R}^{1}\right)+\mathscr{Z}_{R}^{1}$ and $\gamma_{\nu}$ is the zero map.

(3) Let $E$ be the completion of $R$ at its maximal ideal. We define the filtration $\mathrm{F}^{\bullet}$ on $\Omega_{E}^{1} / \mathscr{Z}_{E}^{1}$ in the same way as $\mathrm{F}^{\bullet}\left(\Omega_{R}^{1} / \mathscr{Z}_{R}^{1}\right)$ using $x \in S$. Because $E^{p}$ is faithfully flat over $R^{p}$ and $\mathrm{F}^{\nu}\left(\Omega_{R}^{1} / \mathscr{Z}_{R}^{1}\right) \otimes_{R^{p}} E^{p} \simeq \mathrm{F}^{\nu}\left(\Omega_{E}^{1} / \mathscr{Z}_{E}^{1}\right)$, it suffices to show that $\operatorname{gr}_{\mathrm{F}}^{\nu}\left(\Omega_{E}^{1} / \mathscr{Z}_{E}^{1}\right)=0$ for any $\nu$ with $p \mid \nu$. Let $D$ be the following subring of $E$ :

$$
D:=\mathbb{F}_{q} \llbracket y \rrbracket \subset \mathbb{F}_{q} \llbracket x, y \rrbracket=E,
$$

where $\mathbb{F}_{q}$ denotes the residue field of $E$. Let $\omega=a x^{\nu} d x+b x^{\nu} d y \in \mathrm{F}^{\nu} \Omega_{E}^{1}$ with $a, b \in E$. Take $f \in D$ and $g \in E$ such that $b=f+x g$. Then $\omega \equiv a x^{\nu} d x+f x^{\nu} d y \bmod \mathrm{F}^{\nu+1} \Omega_{E}^{1}$. Since $f \in D=\mathbb{F}_{q} \llbracket y \rrbracket$, we have $d(f d y)=0$ in $\Omega_{E}^{2}$. Hence if $p \mid \nu$, then $f x^{\nu} d y$ belongs to $\mathscr{Z}_{E}$, and

$$
\omega \equiv a x^{\nu} d x \bmod \mathrm{F}^{\nu+1} \Omega_{E}^{1}+\mathscr{Z}_{E}^{1}
$$

Thus (3) follows from the same argument as (2).

We turn to the proof of the inclusion (3.3.3). We compute the map

$$
\pi_{i, m}: \Omega_{R}^{1} / \mathscr{Z}_{R}^{1} \stackrel{\sqrt[3.2 .1]{\longrightarrow}}{\longrightarrow} \operatorname{gr}_{U}^{i} h^{2}\left(A\left[p^{-1}\right]\right) \longrightarrow U^{m i} / U^{m(i+1)} h^{2}\left(B_{m}\left[p^{-1}\right]\right)
$$

using the filtration $\mathrm{F}^{\bullet}$ on $\Omega_{R}^{1} / \mathscr{Z}_{R}^{1}$. For $\nu \geq 0, \pi_{i, m}$ sends $a x^{\nu} d \log (b)+\mathscr{Z}_{R}^{1} \in \mathrm{F}^{\nu}\left(\Omega_{R}^{1} / \mathscr{Z}_{R}^{1}\right)$ with $a \in R$ and $b \in R^{\times}$to

$$
\left\{1+\widetilde{a} x_{m}^{m i+\nu}, \widetilde{b}\right\}+U^{m(i+1)} h^{2}\left(B_{m}\left[p^{-1}\right]\right)
$$

where $\widetilde{a} \in B_{m}$ (resp. $\widetilde{b} \in\left(B_{m}\right)^{\times}$) denotes the residue class of a lift of $a$ to $A$ (resp. the residue class of a lift of $b$ to $A^{\times}$) (cf. Remark 2.6.7). Hence we have

$$
\pi_{i, m}\left(\mathrm{~F}^{\nu}\left(\Omega_{R}^{1} / \mathscr{Z}_{R}^{1}\right)\right) \subset U^{m i+\nu} / U^{m(i+1)} h^{2}\left(B_{m}\left[p^{-1}\right]\right) \text { for } m \geq \nu .
$$

If $m \geq \nu+1$, then $\pi_{i, m}$ induces a map

$$
\varrho_{i, m, \nu}: \operatorname{gr}_{\mathrm{F}}^{\nu}\left(\Omega_{R}^{1} / \mathscr{Z}_{R}^{1}\right) \longrightarrow \operatorname{gr}_{U}^{m i+\nu} h^{2}\left(B_{m}\left[p^{-1}\right]\right)
$$


On the other hand, if $m \geq \nu+2$ and $p \mid(\nu+1)$, then we have

$$
\pi_{i, m}\left(\mathrm{~F}^{\nu+1}\left(\Omega_{R}^{1} / \mathscr{Z}_{R}^{1}\right)\right)=\pi_{i, m}\left(\mathrm{~F}^{\nu+2}\left(\Omega_{R}^{1} / \mathscr{Z}_{R}^{1}\right)\right) \subset U^{m i+\nu+2} / U^{m(i+1)} h^{2}\left(B_{m}\left[p^{-1}\right]\right)
$$

by Lemma 3.3.6(3), so that $\pi_{i, m}$ induces the following map:

$$
\widetilde{\varrho}_{i, m, \nu}: \operatorname{gr}_{\mathrm{F}}^{\nu}\left(\Omega_{R}^{1} / \mathscr{Z}_{R}^{1}\right) \longrightarrow U^{m i+\nu} / U^{m i+\nu+2} h^{2}\left(B_{m}\left[p^{-1}\right]\right) .
$$

By Lemma 3.3.6(3), the inclusion (3.3.3) in question is reduced to the following lemma:

Lemma 3.3.8. Let $\nu$ and $m$ be positive integers with $p \nmid \nu$.

(1) If $m \geq \nu+1$ and $p \nmid(\nu+1)$, then $\varrho_{i, m, \nu}$ is bijective.

(2) If $m \geq \nu+2$ and $p \mid(\nu+1)$, then $\widetilde{\varrho}_{i, m, \nu}$ is bijective.

Proof of Lemma 3.3.8 Put $e_{m}:=m \cdot e, e_{m}^{\prime}:=p e_{m} /(p-1)=m \cdot e^{\prime}$ and $s:=x_{m} \in B_{m}$, for simplicity. Note that $e_{m}$ is the absolute ramification index of $B_{m}$.

(1) Note that $m i+\nu<e_{m}^{\prime}$ and $p \nmid m i+\nu$. Consider the following diagram:

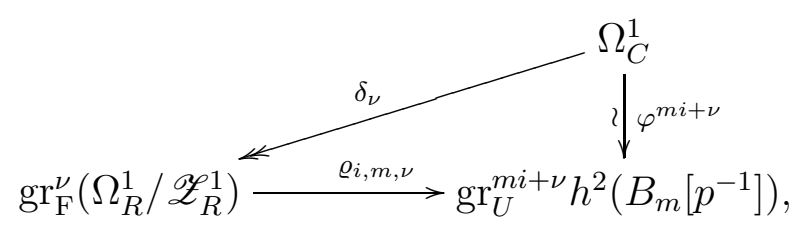

where $\varphi^{m i+\nu}$ is the inverse of the isomorphism (3.2.1) for $B_{m}$ :

$$
\varphi^{m i+\nu}(a \cdot d \log (b))=\left\{1+\widetilde{a} s^{m i+\nu}, \widetilde{b}\right\}+U^{m i+\nu+1} h^{2}\left(B_{m}\left[p^{-1}\right]\right)
$$

with $\widetilde{a} \in B_{m}$ (resp. $\left.\tilde{b} \in\left(B_{m}\right)^{\times}\right)$a lift of $a \in C$ (resp. $\left.b \in C^{\times}\right)$. The arrow $\delta_{\nu}$ is the surjective map induced by the exact sequence (3.3.4) and Lemma 3.3.6(2):

$$
\delta_{\nu}(a \cdot d z)=a^{\prime} \cdot d y+\left(\mathrm{F}^{\nu+1} \Omega_{R}^{1}+\mathscr{Z}_{R}^{1}\right),
$$

where $a^{\prime} \in R$ denotes a lift of $a \in C$. This diagram is obviously commutative, and hence $\varrho_{i, m, \nu}$ is bijective.

(2) Note that $m i+\nu+1<e_{m}^{\prime}$ and $p \mid m i+\nu+1$. The map $\beta_{\nu}: \operatorname{gr}_{\mathrm{F}}^{\nu}\left(\Omega_{R}^{1}\right) \rightarrow \Omega_{C}^{1}$ (cf. (3.3.4)) factors through $\operatorname{gr}_{\mathrm{F}}^{\nu}\left(\Omega_{R}^{1} / \mathscr{Z}_{R}^{1}\right)$ by the following commutative diagram:

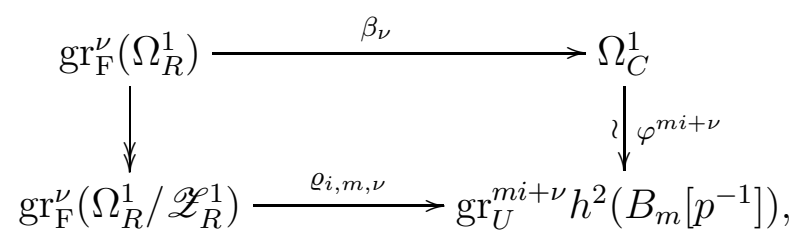

where $\varphi^{m i+\nu}$ is the same as in the proof of (1). Let

$$
\varepsilon_{\nu}: \operatorname{gr}_{\mathrm{F}}^{\nu}\left(\Omega_{R}^{1} / \mathscr{Z}_{R}^{1}\right) \longrightarrow \Omega_{C}^{1}
$$

be the map obtained by factorizing $\beta_{\nu}$. The existence of $\varepsilon_{\nu}$ and the exactness of (3.3.4) imply the exactness of the upper row in the following diagram whose lower row is obviously exact and whose commutativity will be proved below:

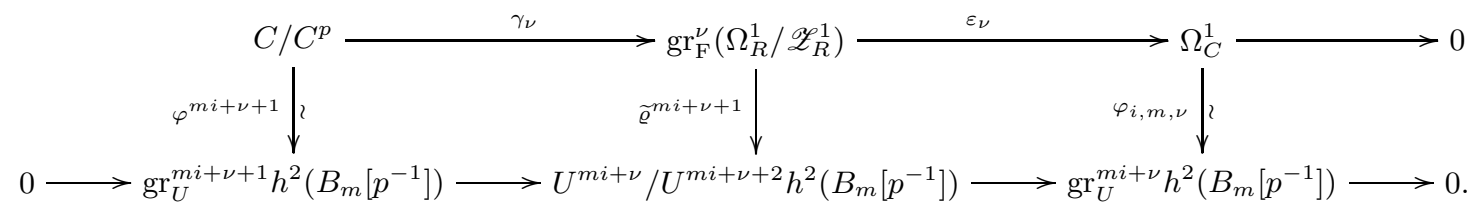


Here $\varphi^{m i+\nu+1}$ is the inverse of the isomorphism (3.2.1) for $B_{m}$ :

$$
\varphi^{m i+\nu+1}(a):=\left\{1+\widetilde{a} s^{m i+\nu+1}, s\right\}+U^{m i+\nu+2} h^{2}\left(B_{m}\left[p^{-1}\right]\right)
$$

with $\widetilde{a} \in B_{m}$ (resp. $\widetilde{b} \in\left(B_{m}\right)^{\times}$) a lift of $a \in C$ (resp. $b \in C^{\times}$) (note that $\Omega_{C}^{1} / \mathscr{Z}_{C}^{1} \subset \Omega_{C}^{2}=$ 0 ). Therefore once we show the commutativity of this diagram, we will have obtained the bijectivity of $\widetilde{\varrho}_{i, m, \nu}$. The commutativity of the right square follows from the construction of $\varepsilon_{\nu}$. We prove the commutativity of the left square for $a \in C / C^{p}$. The case $a=0$ is obvious. Assume that $a \neq 0$. By definition (cf. (3.3.5), (3.3.7)), we have

$$
\widetilde{\varrho}_{i, m, \nu} \circ \gamma_{\nu}(a)=\left\{1+\widetilde{a}(1+s) s^{m i+\nu}, 1+s\right\}+U^{m i+\nu+2} h^{2}\left(B_{m}\left[p^{-1}\right]\right),
$$

where $\widetilde{a} \in B_{m}$ denotes a lift of $a$, and we have used the equality

$$
a x^{\nu} d x=a x^{\nu}(1+x) d \log (1+x) \quad \text { in } \mathrm{F}^{\nu}\left(\Omega_{R}^{1} / \mathscr{Z}_{R}^{1}\right)
$$

(note that $1+x \in R^{\times}$). Let $K_{m}$ be the fraction field of the henselization of $B_{m}$ at the prime ideal $(s)=\left(x_{m}\right)$, and put

$$
h^{2}\left(K_{m}\right):=H^{2}\left(K_{m}, \mu_{p}^{\otimes 2}\right) .
$$

We define the subgroup $U^{m i+\nu+2} h^{2}\left(K_{m}\right) \subset h^{2}\left(K_{m}\right)$ in the same way as for the proof of Corollary 2.6.6, using the prime $s$. Since the natural map

$$
U^{0} / U^{m i+\nu+2} h^{2}\left(B_{m}\left[p^{-1}\right]\right) \longrightarrow h^{2}\left(K_{m}\right) / U^{m i+\nu+2} h^{2}\left(K_{m}\right)
$$

is injective (cf. Proof of Corollary 2.6.6), it suffices to show the congruity relation

$$
\left\{1+\widetilde{a}(1+s) s^{m i+\nu}, 1+s\right\} \equiv\left\{1+\widetilde{a} s^{m i+\nu+1}, s\right\} \bmod U^{m i+\nu+2} h^{2}\left(K_{m}\right)
$$

in $h^{2}\left(K_{m}\right)$. To prove this relation, we first show

$$
\left\{1+\widetilde{a}(1+s) s^{m i+\nu}, 1+s\right\} \equiv-\left\{1+\widetilde{a} s^{m i+\nu+1},-\widetilde{a} s^{m i+\nu}\right\} \bmod U^{m i+\nu+2} h^{2}\left(K_{m}\right) .
$$

Put $c:=m i+\nu$. Indeed, we have

$$
\begin{gathered}
\left\{1+\widetilde{a} s^{c}(1+s), 1+s\right\} \stackrel{(1)}{=}-\left\{1+\widetilde{a} s^{c}(1+s),-\widetilde{a} s^{c}\right\} \stackrel{(2)}{=}-\left\{1+\widetilde{a} s^{c+1}\left(1+\widetilde{a} s^{c}\right)^{-1},-\widetilde{a} s^{c}\right\} \\
\stackrel{(3)}{=}-\left\{1+\widetilde{a} s^{c+1},-\widetilde{a} s^{c}\right\} \bmod U^{c+2} h^{2}\left(K_{m}\right),
\end{gathered}
$$

where (1) and (2) follow from the following equalities in $h^{2}\left(K_{m}\right)$, respectively:

$$
\begin{aligned}
\left(1^{\prime}\right): \quad\{1+v w, v\} & =-\{1+v w,-w\} & & (v, w \neq 0, v w \neq-1), \\
\left(2^{\prime}\right):\{1+w+v,-w\} & =\left\{1+v(1+w)^{-1},-w\right\} & & (w \neq 0,-1, w+v \neq-1)
\end{aligned}
$$

and the congruity (3) follows from Theorem 2.3.3 (2) (note that $c+1<e_{m}^{\prime}$ ). Hence we obtain the congruity (3.3.9) in question again by the equality ( $\left.1^{\prime}\right)$ with $v=s$ and $w=\widetilde{a} s^{m i+\nu}$. Thus we obtain Lemma 3.3.8, 3.3.3) and Proposition 3.3.1(1).

Next we show Proposition 3.3.1(2). Let $R^{\text {sh }}$ be the strict henselization of $R$ at its maximal ideal. We define the filtration $V^{\nu} K_{2}^{M}\left(R^{\mathrm{sh}}[1 / x]\right) \subset K_{2}^{M}\left(R^{\mathrm{sh}}[1 / x]\right)(\nu \geq 1)$ as the subgroup generated by symbols

$$
\left\{1+a x^{\nu}, b\right\} \quad \text { with } a \in R^{\mathrm{sh}} \text { and } b \in\left(R^{\mathrm{sh}}[1 / x]\right)^{\times} .
$$

We define $V^{\bullet}$ on $\Omega_{R, \log }^{2}$ as $V^{0} \Omega_{R, \log }^{2}:=\Omega_{R, \log }^{2}$ and

$$
V^{\nu} \Omega_{R, \log }^{2}:=\Omega_{R, \log }^{2} \cap \operatorname{Im}\left(V^{\nu} K_{2}^{M}\left(R^{\mathrm{sh}}[1 / x]\right) \stackrel{d \log }{\longrightarrow} \Omega_{R^{\operatorname{sh}}[1 / x], \log }^{2}\right) \quad(\nu \geq 1) .
$$


It is easy to see that $V^{\nu} \Omega_{R, \log }^{2} \subset x^{\nu-1} \cdot \Omega_{R}^{2}$ for $\nu \geq 1$, so that we have

$$
\bigcap_{\nu \geq 1} V^{\nu} \Omega_{R, \log }^{2}=0 \text {. }
$$

It suffices to show that the kernel of the map

$$
\prod_{m=2}^{\nu+1} \tau_{0, m}: \Omega_{R, \log }^{2} \longrightarrow \prod_{m=2}^{\nu+1} U^{0} / U^{m} h^{2}\left(B_{m}\left[p^{-1}\right]\right)
$$

is contained in $V^{\nu+1} \Omega_{R, \log }^{2}$ for any $\nu \geq 1$. If $m \geq \nu \geq 0$, the image of the composite map

$$
V^{\nu} \Omega_{R, \log }^{2} \longleftrightarrow \operatorname{gr}_{U}^{0} h^{2}\left(A\left[p^{-1}\right]\right) \longrightarrow U^{0} / U^{m} h^{2}\left(B_{m}\left[p^{-1}\right]\right)
$$

lies in $U^{\nu} / U^{m} h^{2}\left(B_{m}\left[p^{-1}\right]\right)$, which induces the following map:

$$
\sigma_{m, \nu}: \operatorname{gr}_{V}^{\nu} \Omega_{R, \log }^{2} \longrightarrow \operatorname{gr}_{U}^{\nu} h^{2}\left(B_{m}\left[p^{-1}\right]\right) \quad \text { if } m>\nu .
$$

Now we are reduced to the following lemma:

Lemma 3.3.10. (1) We have $\operatorname{gr}_{V}^{0} \Omega_{R, \log }^{2}=0$.

(2) If $m>\nu \geq 1$, then $\sigma_{m, \nu}$ is bijective.

Proof of Lemma 3.3.10 (1) We define $V^{\bullet}$ on $\Omega_{R^{\text {sh }}, \log }^{2}$ in the same way as for $V^{\bullet} \Omega_{R, \log }^{2}$. We have $\operatorname{gr}_{V}^{0} \Omega_{R, \log }^{2} \subset \operatorname{gr}_{V}^{0} \Omega_{R^{\text {sh }}, \log }^{2}$ by definition. By a similar argument as for Lemma 2.4.1(2) (see also (2.4.3), Lemma 2.4.4 and Remark 2.4.6), we have

$$
\operatorname{gr}_{V}^{0} \Omega_{R^{\text {sh }, \log }}^{2} \subset \Omega_{R^{\text {sh }} /(x), \log }^{2} .
$$

The group on the right hand side is zero because $R^{\mathrm{sh}} /(x)$ is the strict henselization of a local ring of a smooth curve over a finite field. Thus we obtain $\operatorname{gr}_{V}^{0} \Omega_{R, \log }^{2}=0$.

(2) We first prove the case $p \nmid \nu$. Consider the following commutative diagram:

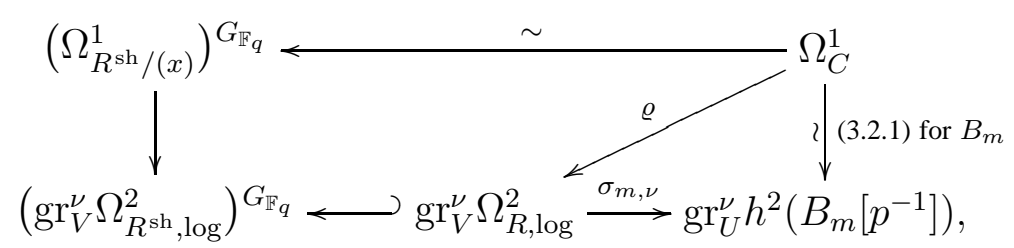

where $\mathbb{F}_{q}$ denotes the residue field of $R$ and the arrow $\varrho$ is the Bloch-Kato map ([BK $\left.],(4.3)\right)$ defined as

$$
a \cdot d \log (b) \mapsto d \log \left(\left\{1+\widetilde{a} \cdot x^{\nu}, \widetilde{b}\right\}\right)+V^{\nu+1} \Omega_{R, \log }^{2}
$$

with $\widetilde{a} \in R$ (resp. $\widetilde{b} \in R^{\times}$) a lift of $a \in R /(x)$ (resp. $\left.b \in(R /(x))^{\times}\right)$. The left vertical arrow is defined in the same way as $\varrho$. In this diagram, the left vertical arrow is bijective by Lemma 2.4.4 and loc. cit., Remark (4.8), which implies the bijectivity of $\varrho$. Hence $\sigma_{m, \nu}$ is bijective as well. If $p \mid \nu$, then noting that $\Omega_{C}^{1} / \mathscr{Z}_{C}^{1}=0$, we have

$$
\operatorname{gr}_{V}^{\nu} \Omega_{R, \log }^{2} \simeq C / C^{p} \simeq \operatorname{gr}_{U}^{\nu} h^{2}\left(B_{m}\left[p^{-1}\right]\right)
$$

by the same argument as before, and $\sigma_{m, \nu}$ is bijective. This completes the proof of Lemma 3.3.10 and Proposition 3.3.1.

Thus Theorem 3.1.1 is reduced to the 2-dimensional case. 
3.4. Proof of the 2-dimensional case. Assume that $\operatorname{dim}(A)=2$, i.e., $A$ is a 2-dimensional excellent henselian regular local ring with finite residue field such that the radical of $(p) \subset A$ is a prime ideal of height 1 . We prove that the specialization map

$$
\operatorname{Br}\left(A\left[p^{-1}\right]\right) \longrightarrow \prod_{v \in Q} \operatorname{Br}(v)
$$

is injective, which will complete the proof of Theorem 3.1.1. We first review the Brauer group theory of $A\left[p^{-1}\right]$ established in [S1], briefly. Let $\mathfrak{p}_{\eta}$ be the radical of $(p) \subset A$. Let $A_{\eta}$ be the henselization of $A$ at $\mathfrak{p}_{\eta}$ and let $K_{\eta}$ be the fraction field of $A_{\eta}$. We regard $K_{\eta}^{\times}$as a topological group in the following way. For a positive integer $n>0$ and an ideal $\mathfrak{I} \subset A$ with $\mathfrak{I} \not \subset \mathfrak{p}_{\eta}$, let $U_{\eta}^{n}(\mathfrak{I})$ be the subgroup of $K_{\eta}^{\times}$generated by

$$
1+\mathfrak{I}\left(\subset A^{\times}\right) \quad \text { and } \quad 1+\mathfrak{p}_{\eta}^{n} A_{\eta}\left(\subset A_{\eta}^{\times}\right) .
$$

We regard $K_{\eta}^{\times}$as a topological group by taking $\left\{U_{\eta}^{n}(\mathfrak{I})\right\}_{n>0, \Im \not \subset \mathfrak{p}_{\eta}}$ as a basis of neighborhoods of $1 \in K_{\eta}^{\times}$(cf. [S1], (2.3)). Let $K$ be the fraction field of $A$. We define the group $\mathscr{C}_{A\left[p^{-1}\right]}$ as

$$
\mathscr{C}_{A\left[p^{-1}\right]}:=\operatorname{Coker}\left(K^{\times} \stackrel{(*)}{\longrightarrow} K_{\eta}^{\times} \oplus \bigoplus_{v \in Q} \mathbb{Z}\right),
$$

where the $v$-component of $(*)$ for $v \in Q$ is the discrete valuation at $v$. We endow $\mathscr{C}_{A\left[p^{-1}\right]}$ with the finest topology that is compatible with its group structure and such that the canonical projection $K_{\eta}^{\times} \rightarrow \mathscr{C}_{A\left[p^{-1}\right]}$ is continuous. Then by [S1], (0.7), Theorem (2.10.1) (see also loc. cit., §3), we have a canonical injective homomorphism

$$
\Phi_{A\left[p^{-1}\right]}: \operatorname{Br}\left(A\left[p^{-1}\right]\right) \longleftrightarrow \operatorname{Hom}_{\text {cont }}\left(\mathscr{C}_{A\left[p^{-1}\right]}, \mathbb{Q} / \mathbb{Z}\right),
$$

where $\operatorname{Hom}_{\text {cont }}(\bullet, \mathbb{Q} / \mathbb{Z})$ denotes the group of all continuous $\mathbb{Q} / \mathbb{Z}$-valued homomorphisms. This map satisfies the following compatibility. For $v \in Q$, let $i_{v}: \mathbb{Z} \rightarrow C_{A\left[p^{-1}\right]}$ be the natural map sending $1 \in \mathbb{Z}$ to $\left(a_{v^{\prime}}\right)_{v^{\prime} \in Q}$ with $a_{v}=1$ and $a_{v^{\prime}}=0$ for $v^{\prime} \neq v$. Then $\Phi_{A\left[p^{-1}\right]}$ fits into the following commutative diagram (see the construction of $\Phi_{A\left[p^{-1}\right]}$ in loc. cit., introduction):

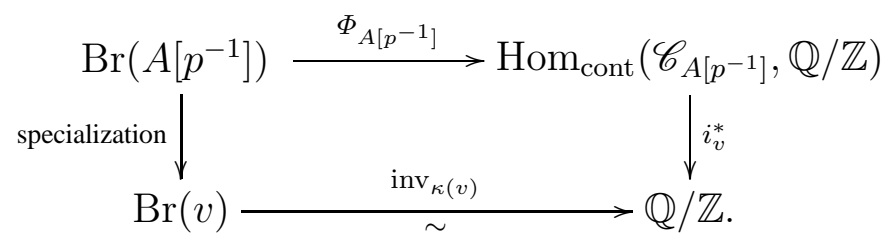

Now suppose that $\omega \in \operatorname{Br}\left(A\left[p^{-1}\right]\right)$ is contained in the kernel of (3.4.1). To show that $\omega=0$, we have only to prove that the continuous character $\Phi_{A\left[p^{-1}\right]}(\omega)$ is zero. By assumption, the pull-back of $\omega$ to $\operatorname{Br}(v)$ is zero for any $v \in Q$, which implies that $\Phi_{A\left[p^{-1}\right]}(\omega)$ factors through

$$
\mathscr{C}_{A\left[p^{-1}\right]} / \bigoplus_{v \in Q} \mathbb{Z} \simeq K_{\eta}^{\times} / K^{\times}
$$

Hence the assertion follows from the continuity of $\Phi_{A\left[p^{-1}\right]}(\omega)$ and the fact that $K^{\times}$is dense in $K_{\eta}^{\times}$, that is, we have $K^{\times} \cdot U_{\eta}^{n}(\mathfrak{I})=K_{\eta}^{\times}$for any integer $n>0$ and any ideal $\mathfrak{I} \subset A$ with $\mathfrak{I} \not \subset \mathfrak{p}_{\eta}$. This completes the proof of Theorem 3.1.1.

Remark 3.4.2. The prime-to- $p$ variant of Corollary 3.1 .2 is stated as follows. Let $k$ be a henselian discrete valuation field with finite residue field $\mathbb{F}$. Let $\mathfrak{o}_{k}$ be the integer ring of $k$ and let $\pi$ be a prime element of $\mathfrak{o}_{k}$. Let $\mathscr{X}$ be a regular scheme which is faithfully flat of finite type over $\operatorname{Spec}\left(\mathfrak{o}_{k}\right)$. Let $Y \subset \mathscr{X}$ be the divisor $\mathscr{X}$ defined by the radical of $(\pi) \subset \mathscr{X}$ 
and let $A$ be a henselian regular local ring obtained by the henselization of $\mathscr{X}$ at a closed point of $Y$ where $Y$ is smooth. For a torsion abelian group $M$, let $M^{\prime}$ be its prime-to-ch $(\mathbb{F})$ torsion part. Then the specialization map

$$
\operatorname{Br}\left(A\left[\pi^{-1}\right]\right)^{\prime} \longrightarrow \prod_{v \in Q} \operatorname{Br}(v)^{\prime}
$$

is injective, where $Q$ denotes the set of all closed points on $\operatorname{Spec}\left(A\left[\pi^{-1}\right]\right)$. This assertion is proved as follows. Let $\mathbb{F}_{q}$ be the residue field of $A$, and let $T \in A$ be a prime element which generates the radical of $(\pi) \subset A$. Put $r:=\operatorname{dim}(A)-1$ and let $\left(T, X_{1}, X_{2}, \ldots, X_{r}\right)$ be a system of regular parameters of $A$. Let $B$ be the residue ring $A /\left(X_{1}, X_{2}, \ldots, X_{r}\right)$. By the absolute purity (cf. Remark 1 .7.2), we have $\operatorname{Br}(A[1 / \pi])^{\prime} \simeq H_{\text {Gal }}^{1}\left(\mathbb{F}_{q}, \mathbb{Q} / \mathbb{Z}\right)^{\prime}$, and the following specialization map is bijective:

$$
\operatorname{Br}\left(A\left[\pi^{-1}\right]\right)^{\prime} \stackrel{\sim}{\longrightarrow} \operatorname{Br}\left(B\left[\pi^{-1}\right]\right)^{\prime} .
$$

Noting that $\operatorname{Spec}(B[1 / \pi])$ is a closed point of $\operatorname{Spec}(A[1 / \pi])$, we obtain the assertion. 


\section{UNRAMIFIEDNESS THEOREM FOR BRAUER GROUPS OF VARIETIES}

Let $k$ be a henselian discrete valuation field of characteristic 0 whose residue field $\mathbb{F}$ is finite and has characteristic $p$. Let $\mathfrak{o}_{k}$ be the integer ring of $k$ and put $S:=\operatorname{Spec}\left(\mathfrak{o}_{k}\right)$. We do not assume that $k$ contains a primitive $p$-th root of unity in this and the later sections.

4.1. Two generalizations on unramifiedness. Let $\mathscr{X}$ be an integral normal scheme which is faithfully flat of finite type over $S$ and such that $X:=\mathscr{X} \otimes_{\mathfrak{o}_{k}} k$ is regular, i.e., smooth over $\operatorname{Spec}(k)$. Let $Y$ be the divisor on $\mathscr{X}$ defined by the radical of $(p) \subset \mathscr{O}_{\mathscr{X}}$. We call $\omega \in \operatorname{Br}(X)$ unramified along $Y$, if $\omega$ is contained in the image of $\operatorname{Br}(\mathscr{X}) \rightarrow \operatorname{Br}(X)$. If $\mathscr{X}$ is regular, this condition is equivalent to that $\omega$ belongs to the subgroup $\operatorname{Br}(\mathscr{X}) \subset \operatorname{Br}(X)$. Following the ideas of Colliot-Thélène-Saito in [CTS], 2 , we introduce two generalized notions of unramifiedness.

Definition 4.1.1. (0) We say that an étale morphism $f: B \rightarrow \mathscr{X}$ is quasi-cs along $Y$, if it satisfies the following two conditions.

(i) For any generic point $\eta$ of $Y$, there exists exactly one connected component $B^{\prime}$ of $B$ which splits completely over $\eta$ (namely, the image of $g:=\left.f\right|_{B^{\prime}}$ contains $\eta$ and $g^{-1}(\eta)$ is isomorphic to the sum of finitely many copies of $\eta$ ).

(ii) Each connected component of $B$ splits completely over some generic point of $Y$.

(1) We say that $\omega \in \operatorname{Br}(X)$ is quasi-unramified along $Y$, if there exists an étale map $B \rightarrow \mathscr{X}$ quasi-cs along $Y$ such that $\left.\omega\right|_{B_{k}} \in \operatorname{Br}\left(B_{k}\right)$ belongs to the image of $\operatorname{Br}(B)$.

(2) We say that $\omega \in \operatorname{Br}(X)$ is 0-unramified, if its specialization $\left.\omega\right|_{v} \in \operatorname{Br}(v)$ is zero for any closed point $v$ on $X$ whose closure in $\mathscr{X}$ is finite over $S$.

Remark 4.1.2. (1) Let $\omega \in \operatorname{Br}(X)$ be unramified along $Y$. Then $\omega$ is quasi-unramified along $Y$ obviously, and we see that $\omega$ is 0 -unramified as follows. Indeed, for a closed point $i: v \rightarrow X$ whose closure in $\mathscr{X}$ is finite over $S$, there is a commutative diagram of schemes

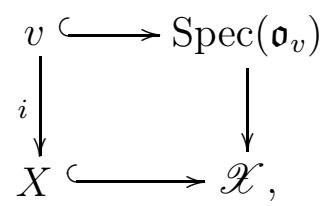

where $\mathfrak{o}_{v}$ is the integer ring of $\kappa(v)$. Hence $\left.\omega\right|_{v}$ is zero by the fact that $\operatorname{Br}\left(\mathfrak{o}_{v}\right)$ is zero.

(2) For a generic point $\eta$ of $Y$, let $A_{\eta}$ be the henselization of $\mathscr{O}_{\mathscr{X}, \eta}$ at its maximal ideal, and let $K_{\eta}$ be the fraction field of $A_{\eta}$. Then we have

$$
\bigoplus_{\eta \in Y^{0}} \operatorname{Br}\left(A_{\eta}\right) \simeq \underset{B}{\underset{\lim }{\longrightarrow}} \operatorname{Br}(B)
$$

where $B$ ranges over all étale $\mathscr{X}$-schemes which are quasi-cs along $Y$ (note that the set of such $B$ 's endowed with a natural semi-order is co-filtered). Hence $\omega \in \operatorname{Br}(X)$ is quasi-unramified along $Y$ if and only if its restriction to $\bigoplus_{\eta \in Y^{0}} \operatorname{Br}\left(K_{\eta}\right)$ belongs to the subgroup $\bigoplus_{\eta \in Y^{0}} \operatorname{Br}\left(A_{\eta}\right)$.

(3) If $\mathscr{X}$ is regular and the purity of Brauer groups holds for $\mathscr{X}$ (in the sense of Definition 1.7.1), then $\omega \in \operatorname{Br}(X)$ is quasi-unramified along $Y$ if and only if $\omega$ is unramified 
along $Y$. Indeed, assuming the purity of Brauer groups, one can easily see that the restriction map

$$
H_{Y}^{3}\left(\mathscr{X}, \mathbb{G}_{\mathrm{m}}\right) \longrightarrow \bigoplus_{\eta \in Y^{0}} H_{\eta}^{3}\left(\mathscr{X}_{\eta}, \mathbb{G}_{\mathrm{m}}\right)
$$

is injective, and that the restriction map

$$
\operatorname{Br}(X) / \operatorname{Br}(\mathscr{X}) \longrightarrow \bigoplus_{\eta \in Y^{0}} \operatorname{Br}\left(K_{\eta}\right) / \operatorname{Br}\left(A_{\eta}\right)
$$

is injective as well.

4.2. Unramifiedness theorem. Let $\mathscr{X}$ and $Y$ be as in 4.1 By Remark 4.1.2(1), the following implications hold for elements of $\operatorname{Br}(X)$ :

$$
\text { quasi-unramified along } Y \Longleftarrow \text { unramified along } Y \Longrightarrow 0 \text {-unramified. }
$$

The main result of this section is the following implication:

Theorem 4.2.1. Any 0-unramified element of $\operatorname{Br}(X)$ is quasi-unramified along $Y$.

Remark 4.2.2. (1) By Remark 4.1.2(2), the assertion in Theorem 4.2.1 is equivalent to the claim that any 0-unramified element maps to zero under the natural map

$$
\operatorname{Br}(X) \longrightarrow \bigoplus_{\eta \in Y^{0}} \operatorname{Br}\left(K_{\eta}\right) / \operatorname{Br}\left(A_{\eta}\right)
$$

(2) $\operatorname{Br}(X)$ is torsion by the regularity of $X$. The prime-to- $p$ part of Theorem 4.2 .1 is due to Colliot-Thélène-Saito [CTS], Théorème 2.1.

By Remark 4.1.2(3), we obtain the following corollary:

Corollary 4.2.3. Assume that $\mathscr{X}$ is regular and that the purity of Brauer groups holds for $\mathscr{X}$. Then the following three conditions for $\omega \in \operatorname{Br}(X)$ are equivalent:

(1) $\omega$ is 0-unramified.

(2) $\omega$ is quasi-unramified along $Y$.

(3) $\omega$ is unramified along $Y$, i.e., belongs to $\operatorname{Br}(\mathscr{X})$.

We will prove the $p$-primary part of Theorem 4.2.1 in $\S 4.3-\$ 4.5$ below. Our proof will proceed as follows. In $\$ 4.3$, we will reduce the problem to the $p$-torsion part; In $\$ 4.4$, it will be further reduced to a special case where $\mathscr{X}$ satisfies certain conditions (see [4.4,2) below). Finally in $\$ 4.5$, we will prove the assertion using Theorem 3.1 .1 . We will often use the following lemma in our proof of Theorem 4.2.1, whose proof is straight-forward and left to the reader:

Lemma 4.2.4. Let $Z$ and $B$ be noetherian normal schemes which are faithfully flat of finite type over $S$, and let $f: B \rightarrow Z$ be an $S$-morphism. Let $\omega \in \operatorname{Br}\left(Z_{k}\right)$ be 0 -unramified. Then $\left.\omega\right|_{B_{k}} \in \operatorname{Br}\left(B_{k}\right)$ is again 0-unramified. 
4.3. Reduction to the $\boldsymbol{p}$-torsion part. For an $S$-scheme $Z$, we put $Z\left[p^{-1}\right]:=Z \otimes_{\mathfrak{o}_{k}} k$. We first prove Theorem 4.2.1 for $p$-primary torsion elements assuming the following:

$(\sharp)$ Theorem 4.2.1 holds for any integral scheme $\mathscr{X}^{\prime}$ étale over $\mathscr{X}$ and any 0 -unramified $\omega \in{ }_{p} \operatorname{Br}\left(\mathscr{X}^{\prime}\left[p^{-1}\right]\right)$ with $p \cdot \omega=0$.

By this step, Theorem 4.2.1 will be reduced to the case of $p$-torsion elements. We proceed by induction on the exponent of the order of $\omega$. Let $\omega \in \operatorname{Br}(X)$ be 0 -unramified with $p^{n} \cdot \omega=0$ $(n \geq 2)$. Put $\theta:=p \cdot \omega$. Clearly, $\theta$ is 0 -unramified. By the induction hypothesis, there exists an étale morphism $f_{1}: \mathscr{X}_{1} \rightarrow \mathscr{X}$ quasi-cs along $Y$ such that the pullback $\theta_{1}:=\left.\theta\right|_{\mathscr{X}_{1}\left[p^{-1}\right]} \in$ $\operatorname{Br}\left(\mathscr{X}_{1}\left[p^{-1}\right]\right)$ belongs to $\operatorname{Br}\left(\mathscr{X}_{1}\right)$. Here let us recall the following fact:

Lemma 4.3.1. Let $O$ be a henselian discrete valuation ring whose residue field $E$ has characteristic $p>0$. Then $\operatorname{Br}(O)$ is p-divisible.

Proof. $\operatorname{Br}(O)$ is isomorphic to $\operatorname{Br}(E)$ (cf. [G], I, Corollaire 6.2, II, Corollaire 2.5) and the last group is $p$-divisible in view of the fact that $\operatorname{cd}_{p}(E)=1$.

By this lemma and the isomorphism (4.1.3) for $\mathscr{X}_{1}$, there exist an étale morphism $f_{2}$ : $\mathscr{X}_{2} \rightarrow \mathscr{X}_{1}$ and an element $\omega^{\prime} \in \operatorname{Br}\left(\mathscr{X}_{2}\right)$ such that $f_{2}$ is quasi-cs along $Y_{1}:=f_{1}^{-1}(Y)$ and such that $\left.\theta_{1}\right|_{\mathscr{X}_{2}\left[p^{-1}\right]}=p \cdot \omega^{\prime}$. Now put $\tau:=\left.\omega\right|_{\mathscr{X}_{2}\left[p^{-1}\right]}-\omega^{\prime} \in \operatorname{Br}\left(\mathscr{X}_{2}\left[p^{-1}\right]\right)$. Because $\tau$ is 0-unramified (cf. Lemma 4.2.4 and Remark 4.1.2(1)) and satisfies

$$
p \cdot \tau=\left.\theta\right|_{\mathscr{X}_{2}\left[p^{-1}\right]}-f_{2}^{*}\left(\theta_{1}\right)=0,
$$

there exists an étale map $f_{3}: \mathscr{X}_{3} \rightarrow \mathscr{X}_{2}$ which is quasi-cs along $f_{2}^{-1}\left(Y_{1}\right)$ and for which $\left.\tau\right|_{\mathscr{X}_{3}\left[p^{-1}\right]} \in \operatorname{Br}\left(\mathscr{X}_{3}\left[p^{-1}\right]\right)$ belongs to $\operatorname{Br}\left(\mathscr{X}_{3}\right)$, where we used $(\sharp)$ for connected components of $\mathscr{X}_{2}$. Finally, let $f: \mathscr{X}_{3} \rightarrow \mathscr{X}$ be the composite of $f_{1}, f_{2}$ and $f_{3}$. Then $\left.\omega\right|_{\mathscr{X}_{3}\left[p^{-1}\right]}$ belongs to $\operatorname{Br}\left(\mathscr{X}_{3}\right)$. Moreover $f$ is quasi-cs along $Y$ or otherwise the disjoint union of étale maps

$$
f^{\prime} \coprod f^{\prime \prime}:\left(\mathscr{X}_{3}\right)^{\prime} \coprod\left(\mathscr{X}_{3}\right)^{\prime \prime} \longrightarrow \mathscr{X}
$$

with $f^{\prime}$ quasi-cs along $Y$. Thus Theorem 4.2.1 is reduced to the $p$-torsion part.

4.4. Reduction to a special case. We show here the following lemma:

Lemma 4.4.1. Let $\mathscr{X}$ and $Y$ be as in 94.1 Then there exists a dense open regular subset $U \subset \mathscr{X}$ which contains all generic points of $Y$.

Proof. Let $\eta$ be a generic point of $Y$. Take a non-empty integral open subset $D_{\eta} \subset Y$ whose generic point is $\eta$ and which is smooth over $\operatorname{Spec}(\mathbb{F})$. Because $\mathscr{X}$ is normal, there exist a dense open subset $V_{\eta}$ of $D_{\eta}$ and a dense open subset $U_{\eta}$ of $\mathscr{X}$ satisfying the following two conditions:

(1) $V_{\eta}$ is a principal Weil divisor on $U_{\eta}$ defined by a prime element of $\Gamma\left(U_{\eta}, \mathscr{O}_{U_{\eta}}\right)$.

(2) $\eta$ is the unique generic point of $U_{\eta} \otimes_{\mathfrak{o}_{k}} \mathbb{F}$.

We show that $U_{\eta}$ is regular at each point on $V_{\eta}$, which implies that $U_{\eta}$ is regular by the condition (2). Let $x$ be a point on $V_{\eta}$ and let $A$ be the local ring $\mathscr{O}_{U_{\eta}, x}=\mathscr{O}_{\mathscr{X}, x}$. Put $c:=$ $\operatorname{dim}(A)$. Using the condition (1) and the smoothness of $V_{\eta}$, one can easily check that the maximal ideal $\mathfrak{m}$ of $A$ is generated by $c$ elements of $A$ as $A$-submodule, which implies that $\operatorname{dim}_{\kappa(x)}\left(\mathfrak{m} / \mathfrak{m}^{2}\right)=c$. Hence $U_{\eta}$ is regular. Now for each $\eta \in Y^{0}$, take a dense open regular subset $U_{\eta} \subset \mathscr{X}$ as above. Then the union $U:=\bigcup_{\eta \in Y^{0}} U_{\eta}$ gives a desired dense open regular subset of $\mathscr{X}$. 
Using the above lemma, we reduce Theorem 4.2.1 to the following case:

(4.4,2) $\mathscr{X}$ is regular, $\zeta_{p} \in \Gamma\left(X, \mathscr{O}_{X}\right)$, and $Y$ is smooth over $\operatorname{Spec}(\mathbb{F})$.

Let $\mathscr{X}$ be a normal scheme as in Theorem 4.2.1 and let $K$ be the function field of $\mathscr{X}$. Let $\mathscr{X}^{\prime}$ be the normalization of $\mathscr{X}$ in $K\left(\zeta_{p}\right)$. Put $X^{\prime}:=\mathscr{X}^{\prime} \otimes_{\mathfrak{o}_{k}} k$. Let $Y^{\prime}$ be the divisor on $\mathscr{X}^{\prime}$ defined by the radical of $(p) \subset \mathscr{O}_{\mathscr{X}}$. Then it is easy to see that the map $X^{\prime} \rightarrow X$ is a finite étale abelian covering whose Galois group $G$ has order prime to $p$ (in fact, $X^{\prime}$ is isomorphic to a scalar extension of $X$ ). By this fact, we obtain isomorphisms

$$
\begin{aligned}
& \operatorname{Br}(X)_{p \text {-tors }} \simeq\left(\operatorname{Br}\left(X^{\prime}\right)_{p \text {-tors }}\right)^{G}, \\
& \operatorname{Br}(X)_{p \text {-tors }}^{0 \text {-ur }} \simeq\left(\operatorname{Br}\left(X^{\prime}\right)_{p \text {-tors }}^{0 \text {-ur }}\right)^{G}, \\
& \operatorname{Br}(X)_{p \text {-tors }}^{\text {qur- } Y} \simeq\left(\operatorname{Br}\left(X^{\prime}\right)_{p \text {-tors }}^{\text {qur- } Y^{\prime}}\right)^{G},
\end{aligned}
$$

where $\operatorname{Br}(X)^{0 \text {-ur }}$ and $\operatorname{Br}\left(X^{\prime}\right)^{0 \text {-ur }}$ denote the subgroups of 0 -unramified elements. $\operatorname{Br}(X)^{\text {qur- } Y}$ (resp. $\operatorname{Br}\left(X^{\prime}\right)^{\text {qur- } Y^{\prime}}$ ) denotes the subgroup of elements which are quasi-unramified along $Y$ (resp. along $Y^{\prime}$ ). Hence Theorem 4.2.1 for $\mathscr{X}$ is reduced to that for $\mathscr{X}^{\prime}$. By Lemma 4.4.1, there exists a dense open regular subset $U \subset \mathscr{X}^{\prime}$ which contains all generic points of $Y^{\prime}$ and for which $Y^{\prime} \cap U$ is smooth over $\operatorname{Spec}(\mathbb{F})$. It is easy to see that Theorem 4.2.1 for $\mathscr{X}^{\prime}$ is reduced to that for $U$. Thus Theorem 4.2.1 is reduced to the situation of (4.4,2).

4.5. End of proof. For a ring or a scheme $Z$, put $\operatorname{br}(Z):={ }_{p} \operatorname{Br}(Z)$. Assuming that $\mathscr{X}$ and $Y$ satisfy $(4.4 .2)$, we prove that any 0 -unramified element of $\operatorname{br}(X)$ is quasi-unramified along $Y$, which will complete our proof of Theorem 4.2.1. For $x \in \mathscr{X}$, let $A_{x}$ be the henselization of the local ring $\mathscr{O}_{\mathscr{X}, x}$ at its maximal ideal, and let $K_{x}$ be the fraction field of $A_{x}$. Now let $Y^{\prime}$ be a connected component of $Y$, which is integral and smooth over $\operatorname{Spec}(\mathbb{F})$ by the assumption (4.4,2). Let $\eta$ be the generic point of $Y^{\prime}$. For a closed point $x$ on $Y^{\prime}$, let $\eta_{x}$ be the point on $\operatorname{Spec}\left(A_{x}\right)$ lying over $\eta$, and let $A_{\eta_{x}}$ be the henselization of $A_{x}$ at $\eta_{x}$. Let $K_{\eta_{x}}$ be the fraction field of $A_{\eta_{x}}$. We prove here the following lemma:

Lemma 4.5.1. The restriction map

$$
\operatorname{br}\left(K_{\eta}\right) / \operatorname{br}\left(A_{\eta}\right) \longrightarrow \prod_{x \in\left(Y^{\prime}\right)_{0}} \operatorname{br}\left(K_{\eta_{x}}\right) / \operatorname{br}\left(A_{\eta_{x}}\right)
$$

is injective. See Remark4.1.2(2) for $A_{\eta}$ and $K_{\eta}$.

Proof. Let $A_{\bar{\eta}}$ be the strict henselization of $A_{\eta}$, and let $K_{\bar{\eta}}$ be the fraction field of $A_{\bar{\eta}}$. Note that the strict henselization of $A_{\eta_{x}}$ coincides with $A_{\bar{\eta}}$. We have a commutative diagram with exact rows

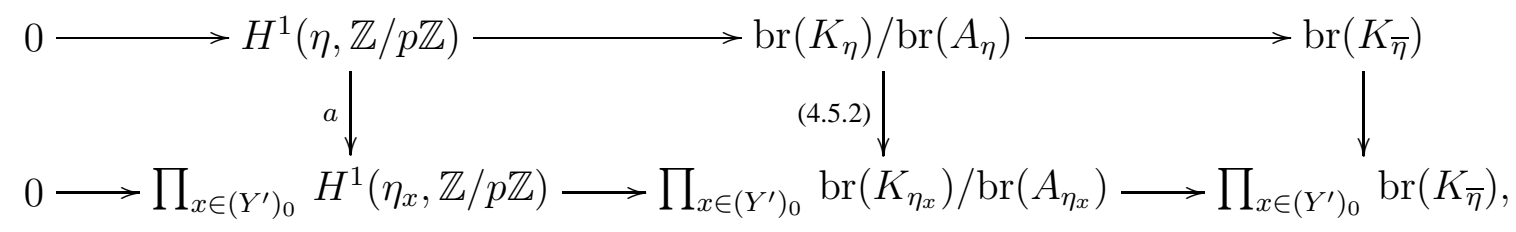

where the vertical arrows are restriction maps, and the exactness of each row follows from a similar argument to Lemma 2.6.4(1). The right vertical map is clearly injective. In what follows, we prove that the left vertical map $a$ is injective, which implies the injectivity of 
(4.5.2). For $x \in\left(Y^{\prime}\right)_{0}$, put $Y_{x}:=\operatorname{Spec}\left(\mathscr{O}_{Y^{\prime}, x}^{\mathrm{h}}\right)$ where $\mathscr{O}_{Y^{\prime}, x}^{\mathrm{h}}$ denotes the henselization of $\mathscr{O}_{Y^{\prime}, x}$ at its maximal ideal. Consider the following commutative diagram with exact rows:

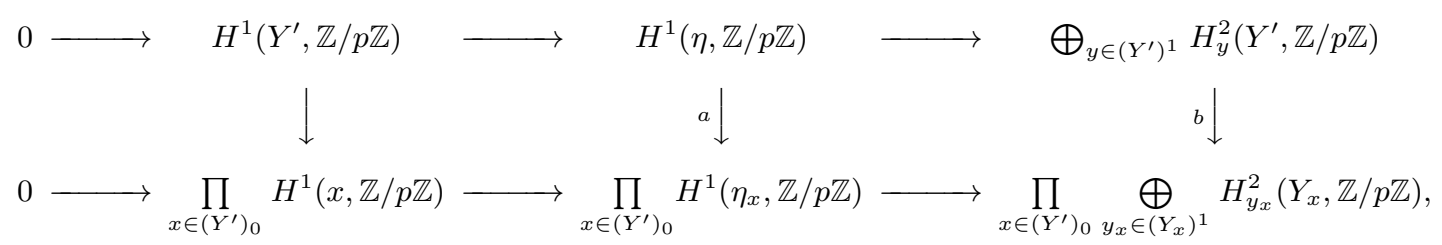

where the vertical arrows are natural restriction maps, and the upper row is obtained from the spectral sequence

$$
E_{1}^{u, v}=\bigoplus_{y \in\left(Y^{\prime}\right)^{u}} H_{y}^{u+v}\left(Y^{\prime}, \mathbb{Z} / p \mathbb{Z}\right) \Longrightarrow H^{u+v}\left(Y^{\prime}, \mathbb{Z} / p \mathbb{Z}\right)
$$

and the fact that $E_{1}^{u, v}=0$ for $(u, v)$ with $v \leq 0$ and $u \geq 1$ (cf. [Gr] , II, (3.5.3)). The lower row is obtained from a similar argument for $\bar{Y}_{x}$ together with the isomorphism

$$
H^{1}\left(Y_{x}, \mathbb{Z} / p \mathbb{Z}\right) \simeq H^{1}(x, \mathbb{Z} / p \mathbb{Z}) .
$$

In this diagram, the left vertical arrow is injective by the Čebotarev density theorem [Se2], Theorem 7. The injectivity of the arrow $b$ is checked as follows. We have

$$
H_{y}^{2}\left(Y^{\prime}, \mathbb{Z} / p \mathbb{Z}\right) \simeq H^{0}\left(y, R^{2} i_{y}^{!} \mathbb{Z} / p \mathbb{Z}\right)
$$

for any $y \in\left(Y^{\prime}\right)^{1}$ with $i_{y}: y \rightarrow Y$, and we have

$$
H_{y_{x}}^{2}\left(Y_{x}, \mathbb{Z} / p \mathbb{Z}\right) \simeq H^{0}\left(y_{x}, R^{2} i_{y_{x}}^{!} \mathbb{Z} / p \mathbb{Z}\right)
$$

for any $x \in\left(Y^{\prime}\right)_{0}$ and any $y_{x} \in\left(Y_{x}\right)^{1}$ with $i_{y_{x}}: y_{x} \rightarrow Y_{x}$. Hence $b$ is the restriction map by pro-finite étale morphisms on groups of global sections of étale sheaves, which is injective. Thus $a$ is injective as well and we obtain the lemma.

We turn to the proof of Theorem 4.2.1. For a $k$-algebra $R$ containing a primitive $p$-th root of unity $\zeta_{p}$, put $h^{2}(R):=H^{2}\left(R, \mu_{p}^{\otimes 2}\right)$ and define

$$
\operatorname{br}(R)^{\mathrm{sym}}:=\operatorname{Im}\left(K_{2}^{M}(R) \longrightarrow h^{2}(R) \stackrel{(*)}{\longrightarrow} \operatorname{br}(R)\right),
$$

where $(*)$ is given by the composite map

$$
h^{2}(R)=H^{2}\left(R, \mu_{p}\right) \otimes \mu_{p} \stackrel{a \otimes \zeta_{p} \mapsto a}{\sim} H^{2}\left(R, \mu_{p}\right) \longrightarrow \mathrm{br}(R) .
$$

Now we finish the proof of Theorem 4.2.1 as follows. Let $\omega \in \operatorname{br}(X)$ be 0 -unramified. Our task is to show that its value under the natural map

$$
\operatorname{br}(X) \longrightarrow \bigoplus_{\eta \in Y^{0}} \operatorname{br}\left(K_{\eta}\right) / \mathrm{br}\left(A_{\eta}\right)
$$

is zero (cf. Remark4.2.2 (1), Lemma4.3.1). We use here the following trick. By the assumption (4.4.2), we have $\operatorname{br}\left(K_{\eta}\right) \simeq h^{2}\left(K_{\eta}\right)$. Any element of $h^{2}\left(K_{\eta}\right)$ is represented by an element of $K_{2}^{M}\left(K_{\eta}\right)$ by the Merkur'ev-Suslin theorem [MS], and we have

$$
\bigoplus_{\eta \in Y^{0}} K_{2}^{M}\left(K_{\eta}\right) \simeq \underset{B}{\lim _{B}} K_{2}^{M}\left(\Gamma\left(B, \mathscr{O}_{B}\right)\left[p^{-1}\right]\right)
$$

where $B$ runs through all affine schemes which are étale over $\mathscr{X}$ and quasi-cs along $Y$ (cf. (4.1.3). Hence replacing $\mathscr{X}$ by a suitable affine scheme étale over $\mathscr{X}$ and using Lemma 
4.2.4, we may suppose that the given 0 -unramified $\omega \in \operatorname{br}(X)$ is contained in $\operatorname{br}(X)^{\mathrm{sym}}$. Consider the following commutative diagram of restriction maps:

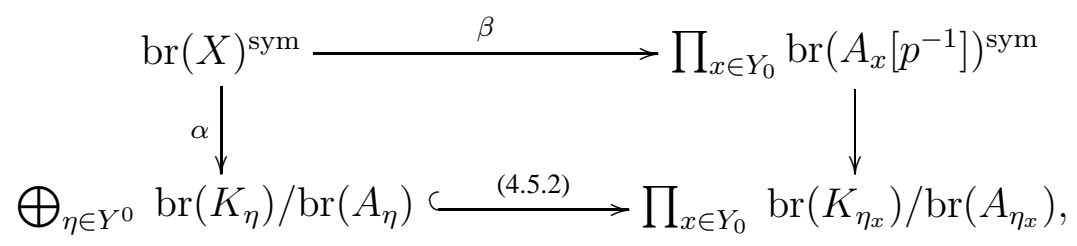

where $\alpha$ and $\beta$ are natural maps. By a variant of Lemma 4.2.4, $\beta(\omega)$ is contained in

$$
\prod_{x \in Y_{0}} \operatorname{Ker}\left(\operatorname{br}\left(A_{x}\left[p^{-1}\right]\right)^{\mathrm{sym}} \rightarrow \prod_{v \in \operatorname{Spec}\left(A_{x}\left[p^{-1}\right]\right)_{0}} \operatorname{br}(v)\right)
$$

which is zero by Theorem 3.1 .1 and the fact that

$$
\operatorname{br}\left(A_{x}\left[p^{-1}\right]\right)^{\mathrm{sym}} \subset \operatorname{br}^{\mathrm{a}}\left(A_{x}\left[p^{-1}\right]\right) \quad \text { for any } \quad x \in Y_{0}
$$

(cf. Remark 2.6.7). Thus $\alpha(\omega)$ is zero. This completes the proof of Theorem 4.2.1,

4.6. Application to arithmetic schemes. Let $\mathscr{X}$ be an integral regular scheme which is proper flat of finite type over $\operatorname{Spec}(\mathbb{Z})$. Put $X:=\mathscr{X} \otimes_{\mathbb{Z}} \mathbb{Q}$.

Theorem 4.6.1. Assume that the purity of Brauer groups holds for $\mathscr{X}$. Then there is an exact sequence:

$$
0 \longrightarrow \operatorname{Br}(\mathscr{X}) \longrightarrow \operatorname{Br}(X) \longrightarrow \prod_{x \in X_{0}} \operatorname{Br}(x) / \operatorname{Br}\left(\mathfrak{o}_{x}\right),
$$

where for $x \in X_{0}, \mathfrak{o}_{x}$ denotes the integral closure of $\mathbb{Z}$ in $\kappa(x)$.

Remark 4.6.2. For $x \in X_{0}, \operatorname{Br}\left(\mathfrak{o}_{x}\right)$ is a finite 2-torsion group by the classical Hasse principle.

Proof. The above sequence is a complex by the properness of $\mathscr{X}$. We show that the resulting specialization map

$$
\operatorname{Br}(X) / \operatorname{Br}(\mathscr{X}) \longrightarrow \prod_{x \in X_{0}} \operatorname{Br}(x) / \operatorname{Br}\left(\mathfrak{o}_{x}\right)
$$

is injective. Let $P$ be the set of all prime numbers. For $p \in P$, let $\mathbb{Z}_{p}^{\text {h }}$ be the henselization of $\mathbb{Z}$ at $(p)$. Put $\mathbb{Q}_{p}^{\mathrm{h}}:=\operatorname{Frac}\left(\mathbb{Z}_{p}^{\mathrm{h}}\right)$, and let $Q_{p}$ be the set of all closed points on $X_{\mathbb{Q}_{p}^{\mathrm{h}}}$. We construct the map

$$
\alpha: \prod_{x \in X_{0}} \operatorname{Br}(x) / \operatorname{Br}\left(\mathfrak{o}_{x}\right) \longrightarrow \prod_{p \in P} \prod_{v \in Q_{p}} \operatorname{Br}(v)
$$

as follows. Let $x$ be a closed point on $X$ and let $v$ be a closed point on $X_{\mathbb{Q}_{p}^{\text {h }}}$. We define the $(x, v)$-component of $\alpha$ as the natural restriction map (resp. the zero map), if the composite map $v \rightarrow X_{\mathbb{Q}_{p}^{\mathrm{h}}} \rightarrow X$ factors through $x \rightarrow X$ (resp. otherwise). Note that for $v \in Q_{p}$, there exists a unique $x \in X_{0}$ such that the composite map $v \rightarrow X_{\mathbb{Q}_{p}^{\mathrm{h}}} \rightarrow X$ factors through $x \rightarrow X$, and that this uniqueness implies the well-definedness of $\alpha$. Now let us consider a commutative diagram of specialization maps

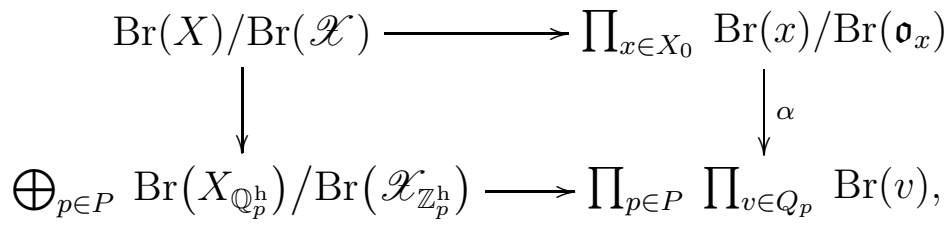


whose commutativity follows from the definition of $\alpha$. In this diagram, the bottom horizontal arrow is injective by Corollary 4.2.3, and the left vertical arrow is injective by the localization exact sequences

$$
\begin{gathered}
\cdots \longrightarrow \operatorname{Br}(\mathscr{X}) \longrightarrow \operatorname{Br}(X) \longrightarrow \bigoplus_{p \in P} H_{Y_{p}}^{3}\left(\mathscr{X}, \mathbb{G}_{\mathrm{m}}\right) \longrightarrow \cdots, \\
\cdots \longrightarrow \operatorname{Br}\left(\mathscr{X}_{\mathbb{Z}_{p}^{\mathrm{h}}}\right) \longrightarrow \operatorname{Br}\left(X_{\mathbb{Q}_{p}^{\mathrm{h}}}\right) \longrightarrow H_{Y_{p}}^{3}\left(\mathscr{X}_{\mathbb{Z}_{p}^{\mathrm{h}}}, \mathbb{G}_{\mathrm{m}}\right) \longrightarrow \cdots,
\end{gathered}
$$

with $Y_{p}:=\mathscr{X} \otimes_{\mathbb{Z}} \mathbb{F}_{p}$, and the excision isomorphism

$$
H_{Y_{p}}^{3}\left(\mathscr{X}, \mathbb{G}_{\mathrm{m}}\right) \simeq H_{Y_{p}}^{3}\left(\mathscr{X}_{\mathbb{Z}_{p}^{\mathrm{h}}}, \mathbb{G}_{\mathrm{m}}\right) \text { for each } p \in P .
$$

Hence the upper horizontal map is injective as well and we obtain the theorem. 


\section{ZERO-CYCLES ON CUBIC SURFACES}

In this section, we compute $A_{0}$ of cubic surfaces explicitly using the unramifiedness theorem proved in the previous section.

5.1. Setting and results. Let $k, \mathfrak{o}_{k}, \mathbb{F}$ and $p$ be as in the beginning of $₫ 4$. Let $a$ be an element of $k^{\times}$which is not a cube in $k$. We are concerned with a cubic surface

$$
X:=\left\{T_{0}^{3}+T_{1}^{3}+T_{2}^{3}+a T_{3}^{3}=0\right\} \subset \operatorname{Proj}\left(k\left[T_{0}, T_{1}, T_{2}, T_{3}\right]\right)=\mathbb{P}_{k}^{3} .
$$

Let $\zeta_{3}$ be a primitive cubic root of unity in $\bar{k}$.

Theorem 5.1.1. (1) Assume $p \neq 3$. Then we have

$$
A_{0}(X) \simeq\left\{\begin{array}{cl}
0 & \text { if } \operatorname{ord}_{k}(a) \equiv 0 \bmod 3, \\
\mathbb{Z} / 3 & \text { if } \operatorname{ord}_{k}(a) \neq 0 \bmod 3 \text { and } \zeta_{3} \notin k, \\
\mathbb{Z} / 3 \oplus \mathbb{Z} / 3 & \text { if } \operatorname{ord}_{k}(a) \neq \equiv \bmod 3 \text { and } \zeta_{3} \in k .
\end{array}\right.
$$

(2) Assume $p=3 \operatorname{ord}_{k}(a) \equiv 1 \bmod 3$ and $\zeta_{3} \in k$. Then we have

$$
A_{0}(X) \simeq \mathbb{Z} / 3 \oplus \mathbb{Z} / 3
$$

(1) is stated in [CTS], Example 2.8 under a slightly simpler setting. We include a proof of (1) here for the convenience of the reader. (2) is a new result and would be the first example of a potentially rational surface which splits over a wildly ramified extension and whose $A_{0}$ is computed explicitly. It would be interesting to find cycles which generate $A_{0}(X)$ in the theorem.

To prove Theorem 5.1.1, we need the following three facts, where $X$ is as before.

Proposition 5.1.2 (Colliot-Thélène). The following map induced by the Brauer-Manin pairing is injective:

$$
A_{0}(X) \longrightarrow \operatorname{Hom}(\operatorname{Br}(X) / \operatorname{Br}(k), \mathbb{Q} / \mathbb{Z}) .
$$

Proof. The case $X(k) \neq \emptyset$ is stated in [CT1], Proposition 5. Otherwise, the assertion follows from his injectivity result in loc. cit., Proposition 7 (b) and the same arguments as in loc. cit., Proposition 5 (cf. [B]1], Theorem (2.1), Proposition (A.1)). See also [S2], Theorem A, [CT2], Théorème 8.4 , and [ $[\mathrm{Kh}]$, p. 70, Corollaire 2 for generalizations.

Proposition 5.1.3. Let $\mathscr{U}$ be a regular scheme which is faithfully flat over $S$ and satisfies $\mathscr{U} \otimes_{\mathfrak{o}_{k}} k \simeq X$. Let $\eta$ be a generic point of $\mathscr{U} \otimes_{\mathfrak{o}_{k}} \mathbb{F}$, let $A_{\eta}$ be the henselization of $\mathscr{O}_{\mathscr{U}, \eta}$ and let $K_{\eta}$ be the fraction field of $A_{\eta}$. Assume that

$$
\iota^{-1}\left(\operatorname{Br}\left(A_{\eta}\right)\right)=0, \quad \text { where } \iota: \operatorname{Br}(X) \rightarrow \operatorname{Br}\left(K_{\eta}\right) .
$$

Then the following map induced by the Brauer-Manin pairing is surjective:

$$
A_{0}(X) \longrightarrow \operatorname{Hom}(\operatorname{Br}(X) / \operatorname{Br}(k), \mathbb{Q} / \mathbb{Z}),
$$

Proof. By (5.1.4), Theorem 4.2.1 and Remark 4.1.2(2), the map in question has dense image. As we mentioned in $1.2, \operatorname{Br}\left(X_{L}\right) / \operatorname{Br}(L)$ is zero for $L=k(\sqrt[3]{a})$. Hence $\operatorname{Br}(X) / \operatorname{Br}(k)$ is a finite 3 -torsion and the assertion follows.

To state the third fact, we assume that $k$ contains $\zeta_{3}$, and fix an isomorphism

$$
{ }_{3} \operatorname{Br}(k(X)) \simeq H^{2}\left(k(X), \mu_{3}^{\otimes 2}\right), \quad x \mapsto x \otimes \zeta_{3},
$$


where $k(X)$ is the function field of $X$. Consider rational functions

and put

$$
f:=\frac{T_{0}+\zeta_{3} T_{1}}{T_{0}+T_{1}}, g:=\frac{T_{0}+T_{2}}{T_{0}+T_{1}} \in k(X)^{\times}
$$

$$
\boldsymbol{e}_{1}=(a, f)_{\zeta_{3}}, \boldsymbol{e}_{2}=(a, g)_{\zeta_{3}} \in{ }_{3} \operatorname{Br}(k(X)),
$$

where for $u, v \in k(X)^{\times},(u, v)_{\zeta_{3}}$ denotes the inverse image of $\{u, v\} \in H^{2}\left(k(X), \mu_{3}^{\otimes 2}\right)$ under the isomorphism (5.1.5).

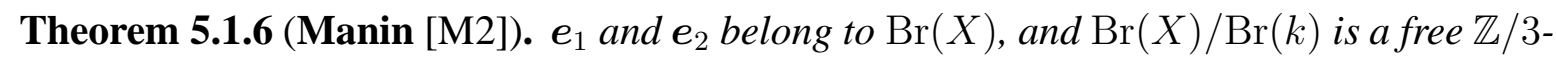
module of rank 2 generated by $\boldsymbol{e}_{1}$ and $\boldsymbol{e}_{2}$.

5.2. Proof of Theorem 5.1.1(1). Without loss of generality, we may assume that $a \in \mathfrak{o}_{k}-$ $\{0\}$ with $\operatorname{ord}_{k}(a)=0,1$ or 2 . Consider a projective flat model of $X$ over $S:=\operatorname{Spec}\left(\mathfrak{o}_{k}\right)$

$$
\mathscr{X}=\operatorname{Proj}\left(\mathfrak{o}_{k}\left[T_{0}, T_{1}, T_{2}, T_{3}\right] /\left(T_{0}^{3}+T_{1}^{3}+T_{2}^{3}+a T_{3}^{3}\right)\right) \subset \mathbb{P}_{S}^{3} .
$$

Let $\eta$ be the generic point of $Y:=\mathscr{X} \otimes_{\mathfrak{o}_{k}} \mathbb{F}$, let $A_{\eta}$ be the henselization of $\mathscr{O}_{\mathscr{X}, \eta}$ and let $K_{\eta}$ be the fraction field of $A_{\eta}$. We divide the problem into 4 cases as follows.

Case (i): $p \neq 3, a \in \mathfrak{o}_{k}^{\times}$and $\zeta_{3} \in k$. In this case it is easy to see that $\mathscr{X}$ is smooth over $S$. Once we show

$$
\operatorname{Br}(X)=\operatorname{Br}(\mathscr{X})+\operatorname{Im}(\operatorname{Br}(k) \rightarrow \operatorname{Br}(X)),
$$

then $A_{0}(X)=0$ by Proposition 5.1.2. Since we have

$$
\operatorname{Br}(\mathscr{X})=\operatorname{Ker}\left(\operatorname{Br}(X) \rightarrow \operatorname{Br}\left(K_{\eta}\right) / \operatorname{Br}\left(A_{\eta}\right)\right),
$$

by the purity of Brauer groups for $\mathscr{X}$, it is enough to show

$$
\operatorname{Im}\left(\operatorname{Br}(X) \rightarrow \operatorname{Br}\left(K_{\eta}\right)\right) \subset \operatorname{Br}\left(A_{\eta}\right)+\operatorname{Im}\left(\operatorname{Br}(k) \rightarrow \operatorname{Br}\left(K_{\eta}\right)\right),
$$

in order to show (5.2.2). Since $p \neq 3$ and $\zeta_{3} \in k$, there is an exact sequence

$$
0 \longrightarrow{ }_{3} \operatorname{Br}\left(A_{\eta}\right) \otimes \mu_{3}(k) \longrightarrow H^{2}\left(k(X), \mu_{3}^{\otimes 2}\right) \stackrel{\delta}{\longrightarrow} \kappa(\eta)^{\times} / 3 \longrightarrow 0
$$

and it is easy to check $\delta\left(\boldsymbol{e}_{i} \otimes \zeta_{3}\right)=0$ for $i=1,2$, which implies (5.2.3) by Theorem 5.1.6.

Case (ii): $p \neq 3, a \in \mathfrak{o}_{k}^{\times}$and $\zeta_{3} \notin k$. In this case, the assertion is reduced to the case (i) immediately by a standard norm argument.

Case (iii): $p \neq 3 \operatorname{ord}_{k}(a)=1,2$ and $\zeta_{3} \in k$. One can easily check that the fixed model $\mathscr{X}$ is regular at $\eta \in Y$, i.e., $\mathscr{O}_{\mathscr{X}, \eta}$ is a discrete valuation ring. By Propositions 5.1.2, 5.1.3 and Theorem 5.1.6, we have only to show

$$
\iota^{-1}\left(\operatorname{Br}\left(A_{\eta}\right)\right)=0, \quad \text { with } \iota: \operatorname{Br}(X) \rightarrow \operatorname{Br}\left(K_{\eta}\right) .
$$

Note that we have the exact sequence (5.2.4) in this case as well. For

$$
\boldsymbol{\omega}=\alpha \boldsymbol{e}_{1}+\beta \boldsymbol{e}_{2} \in{ }_{3} \operatorname{Br}(X) \subset{ }_{3} \operatorname{Br}(k(X)) \quad(\alpha, \beta \in \mathbb{Z} / 3),
$$

we have

$$
\delta\left(\boldsymbol{\omega} \otimes \zeta_{3}\right)=\operatorname{ord}_{k}(a) \cdot f^{\alpha} g^{\beta}=\operatorname{ord}_{k}(a) \cdot\left(\frac{T_{0}+\zeta_{3} T_{1}}{T_{0}+T_{1}}\right)^{\alpha}\left(\frac{T_{0}+T_{2}}{T_{0}+T_{1}}\right)^{\beta} \in \kappa(\eta)^{\times} / 3,
$$

where we regarded $f$ and $g$ as rational functions on $Y$. Thus it is enough to show 
Lemma 5.2.5. For $\alpha, \beta \in \mathbb{Z}$, assume that $f^{\alpha} g^{\beta} \in \kappa(\eta)^{\times}$belongs to $\left(\kappa(\eta)^{\times}\right)^{3}$. Then we have $\alpha \equiv \beta \equiv 0 \bmod 3$.

Proof. Let $E$ be the elliptic curve over $\mathbb{F}$ defined as

$$
E:=\operatorname{Proj}\left(\mathbb{F}\left[T_{0}, T_{1}, T_{2}\right] /\left(T_{0}^{3}+T_{1}^{3}+T_{2}^{3}\right)\right) \subset \mathbb{P}_{\mathbb{F}}^{2} .
$$

It is easy to see that $\kappa(\eta)$ is the rational function field in one variable over the function field $\mathbb{F}(E)$. Since $\left(\mathbb{F}(Y)^{\times}\right)^{3} \cap \mathbb{F}(E)^{\times}=\left(\mathbb{F}(E)^{\times}\right)^{3}$ and $f, g \in \mathbb{F}(E)^{\times}$, the assumption of the lemma implies that $f^{\alpha} g^{\beta} \in\left(\mathbb{F}(E)^{\times}\right)^{3}$. We now look at the divisors on $E$

$$
\operatorname{div}_{E}(f)=3([P]-[O]), \quad \operatorname{div}_{E}(g)=3([Q]-[O]),
$$

where we put

$$
O:=(1:-1: 0), P:=\left(1:-\zeta_{3}: 0\right), Q:=(1: 0:-1) .
$$

Take $O$ to be the origin of the elliptic curve $E$, and define zero-cycles $C, C^{\prime}$ on $E$ as $C:=$ $[P]-[O], C^{\prime}:=[Q]-[O]$. Since $f^{\alpha} g^{\beta}=h^{3}$ for some $h \in \kappa(\eta)^{\times}$by assumption, we have

$$
\alpha \cdot C+\beta \cdot C^{\prime}=\operatorname{div}_{E}(h)
$$

as zero-cycles, and the residue class $\alpha \cdot \bar{C}+\beta \cdot \overline{C^{\prime}}$ is zero in $A_{0}(E) \simeq E(\mathbb{F})$. Hence the assertion follows from the linear independence of $\bar{C}$ and $\overline{C^{\prime}}$ in the $\mathbb{Z} / 3$-vector space ${ }_{3} E(\mathbb{F})$.

Case (iv): $p \neq 3, \operatorname{ord}_{k}(a)=1,2$ and $\zeta_{3} \notin k$. Consider the scalar extension

$$
\mathscr{X} \otimes_{\mathfrak{o}_{k}} \mathfrak{o}_{L} \longrightarrow \mathscr{X} \quad\left(L:=k\left(\zeta_{3}\right)\right),
$$

which is étale by the assumption $p \neq 3$. Then we have

$$
\iota^{-1}\left(\operatorname{Br}\left(A_{\eta}\right)\right)=0, \quad \text { with } \iota: \operatorname{Br}(X) \rightarrow \operatorname{Br}\left(K_{\eta}\right)
$$

by the previous case and a standard norm argument. Therefore by Propositions 5.1.2 and 5.1.3, the assertion is reduced to the following proposition due to Colliot-Thélène [CT4], which holds without the assumption $p \neq 3$ :

Proposition 5.2.6 (Colliot-Thélène). Let $\boldsymbol{e}_{i}(i=1,2)$ be the elements of $\operatorname{Br}\left(X_{L}\right)$ in Proposition 5.1.6. Then $\operatorname{Br}(X) / \operatorname{Br}(k)$ is a free $\mathbb{Z} / 3$-module of rank 1 generated by $\operatorname{Cores}_{X_{L} / X}\left(\boldsymbol{e}_{1}\right)$, where Cores $X_{L} / X$ denotes the corestriction map $\operatorname{Br}\left(X_{L}\right) \rightarrow \operatorname{Br}(X)$.

Proof. Put $G:=\operatorname{Gal}(L / k)$, which has order 2. Let $\sigma$ be the generator of $G$. We prove

$$
\sigma\left(\boldsymbol{e}_{1}\right)=\boldsymbol{e}_{1} \quad \text { and } \quad \sigma\left(\boldsymbol{e}_{2}\right)=-\boldsymbol{e}_{2} \quad \text { in }{ }_{3} \operatorname{Br}\left(X_{L}\right),
$$

which implies the assertion by a standard norm argument. To prove (5.2.7), we work with Galois cohomology groups of the function field

$$
F:=L\left(X_{L}\right)=k(X)\left(\zeta_{3}\right) .
$$

Since ${ }_{3} \operatorname{Br}\left(X_{L}\right) \subset H^{2}\left(F, \mu_{3}\right)$, it is enough to show the following two claims:

(1) For $u, v \in F^{\times}$, we have $\sigma\left((u, v)_{\zeta_{3}}\right)=-(\sigma(u), \sigma(v))_{\zeta_{3}}$ in $H^{2}\left(F, \mu_{3}\right)$.

(2) We have

$$
\left\{a, \frac{T_{0}+\zeta_{3}^{-1} T_{1}}{T_{0}+T_{1}}\right\}=-\left\{a, \frac{T_{0}+\zeta_{3} T_{1}}{T_{0}+T_{1}}\right\} \quad \text { in } \quad H^{2}\left(F, \mu_{3}^{\otimes 2}\right) .
$$


We first show (1). Since $\mathbb{Z} / 3 \simeq \mu_{3}^{\otimes 2}$ as $G_{k}$-modules, we have

$$
\begin{aligned}
\sigma\left((u, v)_{\zeta_{3}}\right) & =\sigma\left(\{u, v\} \otimes \zeta_{3}\right)=\{\sigma(u), \sigma(v)\} \otimes \sigma\left(\zeta_{3}\right) \\
& =\{\sigma(u), \sigma(v)\} \otimes \zeta_{3}^{-1}=-(\sigma(u), \sigma(v))_{\zeta_{3}} .
\end{aligned}
$$

We next show (2). Take an affine open subset of $X$ as follows:

$$
\left\{x^{3}+y^{3}+z^{3}+a=0\right\} \subset \mathbb{A}_{k}^{3} \quad\left(x=\frac{T_{0}}{T_{3}}, y=\frac{T_{1}}{T_{3}}, z=\frac{T_{2}}{T_{3}}\right) .
$$

Then noting that $H^{2}\left(F, \mu_{3}^{\otimes 2}\right)$ is a 3-torsion, we compute

$$
\begin{aligned}
\left\{a, \frac{x+\zeta_{3}^{-1} y}{x+y}\right\}+\left\{a, \frac{x+\zeta_{3} y}{x+y}\right\} & =\left\{a, \frac{x^{2}-x y+y^{2}}{(x+y)^{2}}\right\}=\left\{a, \frac{x^{3}+y^{3}}{(x+y)^{3}}\right\} \\
& =\left\{a, a-z^{3}\right\}=\left\{\frac{a}{z^{3}}, \frac{a}{z^{3}}-1\right\}=0 .
\end{aligned}
$$

This completes the proof of Proposition 5.2.6 and Theorem 5.1.1(1).

5.3. Proof of Theorem 5.1.1(2). Without loss of generality, we may assume $a=\pi$ (a prime of $\mathfrak{o}_{k}$ ). Let $\mathscr{X}$ be the projective flat model of $X$ over $\mathfrak{o}_{k}$ defined in (5.2.1). We will use the following affine open subset:

$$
U:=\operatorname{Spec}\left(\mathfrak{o}_{k}[x, y, z] /\left(x^{3}+y^{3}+z^{3}+\pi\right)\right) \quad\left(x=\frac{T_{0}}{T_{3}}, y=\frac{T_{1}}{T_{3}}, z=\frac{T_{2}}{T_{3}}\right) .
$$

Let $\mathscr{X}_{s}$ be the special fiber of $\mathscr{X} \rightarrow S$, which is irreducible. Let $Y$ be the reduced part of $\mathscr{X}_{s}$ and let $\eta$ be the generic point $Y$. It is easy to see that we have

$$
U \cap Y=\operatorname{Spec}(\mathbb{F}[x, y, z] /(x+y+z)) .
$$

Put $e:=\operatorname{ord}_{k}(3)$. We show the following lemma.

Lemma 5.3.3. $\mathscr{O}_{\mathscr{X}, \eta}$ is a discrete valuation ring with absolute ramification index $3 e$.

Proof. Put $t=x+y+z$. Then we have

$$
t^{3}+\pi\left(1+\pi^{e-1} u\right)=x^{3}+y^{3}+z^{3}+\pi=0 \quad \text { in } \quad \mathscr{O}_{U, \eta}=\mathscr{O}_{\mathscr{X}, \eta},
$$

where we put

$$
u:=-\epsilon_{1}\left(x^{2}(y+z)+y^{2}(z+x)+z^{2}(x+y)+2 x y z\right) \text { with } \epsilon_{1}:=3 \pi^{-e} \in \mathfrak{o}_{k}^{\times} .
$$

In view of (5.3.2), this implies that $t$ generates the maximal ideal of $\mathscr{O}_{\mathscr{X}, \eta}$ (and that $u$ belongs to $\mathscr{O}_{\mathscr{X}, \eta}^{\times}$). Hence $\mathscr{O}_{\mathscr{X}, \eta}$ is a noetherian one-dimensional local ring with maximal ideal generated by $t$, which is a discrete valuation ring. The ramification index of $\mathscr{O}_{\mathscr{X}, \eta}$ over $\mathfrak{o}_{k}$ is 3 by (5.3.4), which implies that the absolute ramification index of $\mathscr{O}_{\mathscr{X}, \eta}$ is $3 e$.

Let $A_{\eta}$ be the henselization of $\mathscr{O}_{\mathscr{X}, \eta}$ as before, and let $K_{\eta}$ be its fraction field. Put $H:=$ $H^{2}\left(K_{\eta}, \mu_{3}^{\otimes 2}\right)$ in what follows. Since $k$ contains $\zeta_{3}$, we have

$$
{ }_{3} \operatorname{Br}\left(A_{\eta}\right) \otimes \mu_{3}(k) \subset U^{3 e^{\prime}} H
$$


by Lemma 2.6.4(1), where $U^{*} H$ denotes the filtration on $H$ defined in $\$ 2.6$ and $e^{\prime}$ denotes $p e /(p-1)=3 e / 2$. As before, by Propositions 5.1.2, 5.1.3 and Theorem 5.1.6, we ought to show

$$
\iota^{-1}\left(\operatorname{Br}\left(A_{\eta}\right)\right)=0, \quad \text { with } \iota: \operatorname{Br}(X) \rightarrow \operatorname{Br}\left(K_{\eta}\right) .
$$

Hence it is enough to show the following:

Proposition 5.3.5. Let $\alpha, \beta \in \mathbb{Z} / 3$, and put

$$
\boldsymbol{\omega}:=\alpha \boldsymbol{e}_{1}+\beta \boldsymbol{e}_{2}=\alpha(\pi, f)_{\zeta_{3}}+\beta(\pi, g)_{\zeta_{3}} \in{ }_{3} \operatorname{Br}(X) .
$$

Assume that

$$
\iota(\boldsymbol{\omega}) \otimes \zeta_{3}=\alpha\{\pi, f\}+\beta\{\pi, g\} \in{ }_{3} \operatorname{Br}\left(K_{\eta}\right) \otimes \mu_{3}(k) \simeq H
$$

belongs to $U^{3 e^{\prime}} H$. Then we have $\alpha=\beta=0$, i.e., $\boldsymbol{\omega}=0$.

Note that $\kappa(\eta)=\mathbb{F}(y, z)$ by (5.3.2). By (5.3.4), we have $\pi=-t^{3}\left(1+\pi^{e-1} u\right)^{-1}$ and

$$
\omega=-\alpha\left\{1+\pi^{e-1} u, f\right\}-\beta\left\{1+\pi^{e-1} u, g\right\} \in U^{3(e-1)} H .
$$

One can derive the proposition easily from the following lemma, where the Bloch-Kato isomorphisms (see (2.3.4) and (2.6.3) ) are defined with respect to the prime element $t \in A_{\eta}$.

Lemma 5.3.6. (1) $\left\{1+\pi^{e-1} u, f\right\}$ belongs to $U^{3 e^{\prime}-2} H$, whose residue class in $\operatorname{gr}_{U}^{3 e^{\prime}-2} H \simeq$ $\Omega_{\eta}^{1}$ is zdy-ydz up to the multiplication by a constant in $\mathbb{F}^{\times}$.

(2) $\left\{1+\pi^{e-1} u, g\right\}$ belongs to $U^{3 e-2} H$, whose residue class in $\operatorname{gr}_{U}^{3 e-2} H \simeq \Omega_{\eta}^{1}$ is $z d y-y d z$ up to the multiplication by a constant in $\mathbb{F}^{\times}$.

Proof of Lemma 5.3.6. We first prove (1). Since $x^{3}+y^{3}+z^{3}+\pi=0$, we have

$$
u=\epsilon_{1}\left(y^{2} z+y z^{2}+t v\right) \text { with } v:=-\left(x^{2}+(t-x)^{2}+\pi \cdot t^{-1}\right)
$$

(note that $\pi \cdot t^{-1}$ is contained in the maximal ideal of $A_{\eta}$ ). On the other hand, we have

$$
f=\frac{x+\zeta_{3} y}{x+y}=1+\left(\zeta_{3}-1\right) \frac{y}{x+y}=1+t^{e^{\prime}} \frac{\epsilon_{2} y}{z}+t^{e^{\prime}+1} \frac{\epsilon_{2} y}{z(z-t)},
$$

where $\epsilon_{2}:=\left(1-\zeta_{3}\right) \cdot t^{-e^{\prime}} \in A_{\eta}^{\times}$. We define the filtration $U^{n} K_{\eta}^{\times}(n \geq 0)$ on $K_{\eta}^{\times}$as the full group $K_{\eta}^{\times}$for $n=0$ and the subgroup $\left\{1+t^{n} c \mid c \in A_{\eta}\right\}$ for $n \geq 1$. We recall the following standard facts:

Sublemma 5.3.9. For $a, b \in A_{\eta}, h \in U^{n} K_{\eta}^{\times}(n \geq 0)$ and integers $\ell, m, \nu \geq 1$, we have

$$
\begin{aligned}
& \left\{1+t^{\ell} a+t^{m} b, h\right\} \equiv\left\{1+t^{\ell} a, h\right\}+\left\{1+t^{m} b, h\right\} \bmod U^{\ell+m+n} H \\
& \left\{1+t^{\ell} a\left(1+t^{m} b\right)^{ \pm \nu}, h\right\} \equiv\left\{1+t^{\ell} a, h\right\} \bmod U^{\ell+m+n} H \\
& \left\{1+t^{\ell} a, 1+t^{m} b\right\} \equiv-\left\{1+\frac{t^{\ell+m} a b}{1+t^{\ell} a},-t^{\ell} a\right\} \bmod U^{\ell+2 m} H .
\end{aligned}
$$

Proof of Sublemma 5.3.9 (1) follows from the equality

$$
\frac{\left(1+t^{m} a\right)\left(1+t^{n} b\right)}{1+t^{m} a+t^{n} b}=1+\frac{t^{m+n} a b}{1+t^{m} a+t^{n} b}
$$

and (3) (cf. [BK], Lemma (4.1)). The assertion (2) follows from a similar computation. (3) follows from similar computations as in [Sa2], Lemma 8.7.4. 
We turn to the proof of Lemma 5.3.6(1), and put $\epsilon_{3}:=3 t^{-3 e} \in A_{\eta}^{\times}$. Then we have

$$
\begin{aligned}
& \left\{1+\pi^{e-1} u, f\right\}=\left\{1+\pi^{e-1} \epsilon_{1}\left(y^{2} z+y z^{2}+t v\right), 1+t^{e^{\prime}} \frac{\epsilon_{2} y}{z}+t^{e^{\prime}+1} \frac{\epsilon_{2} y}{z(z-t)}\right\} \\
& \equiv\left\{1+t^{3 e-2} \epsilon_{3} v, 1+t^{e^{\prime}} \frac{\epsilon_{2} y}{z}\right\}+\left\{1+t^{3 e-3} \epsilon_{3}\left(y^{2} z+y z^{2}\right), 1+t^{e^{\prime}+1} \frac{\epsilon_{2} y}{z(z-t)}\right\} \\
& +\left\{1+t^{3 e-3} \epsilon_{3} y^{2} z, 1+t^{e^{\prime}} \frac{\epsilon_{2} y}{z}\right\}+\left\{1+t^{3 e-3} \epsilon_{3} y z^{2}, 1+t^{e^{\prime}} \frac{\epsilon_{2} y}{z}\right\},
\end{aligned}
$$

where the congruity holds modulo $U^{3 e^{\prime}-1} H$ by Sublemma 5.3.9(1)-(3) and (5.3.4). Therefore, it is enough to show

Lemma 5.3.10. (1) The sum

$$
\left\{1+t^{3 e-2} \epsilon_{3} v, 1+t^{e^{\prime}} \frac{\epsilon_{2} y}{z}\right\}+\left\{1+t^{3 e-3} \epsilon_{3}\left(y^{2} z+y z^{2}\right), 1+t^{e^{\prime}+1} \frac{\epsilon_{2} y}{z(z-t)}\right\} \in U^{3 e^{\prime}-2} H
$$

maps to $z d y-y d z$ up to the multiplication by a constant in $\mathbb{F}^{\times}$, under the Bloch-Kato isomorphism $\mathrm{gr}_{U}^{3 e^{\prime}-2} H \simeq \Omega_{\eta}^{1}$ with respect to the prime element $t \in A_{\eta}$.

(2) $\left\{1+t^{3 e-3} \epsilon_{3} y^{2} z, 1+t^{e^{\prime}} \frac{\epsilon_{2} y}{z}\right\}$ belongs to $U^{3 e^{\prime}-1} H$.

(3) $\left\{1+t^{3 e-3} \epsilon_{3} y z^{2}, 1+t^{e^{\prime}} \frac{\epsilon_{2} y}{z}\right\}$ belongs to $U^{3 e^{\prime}} H$.

Proof. (1) Let $\overline{\epsilon_{2} \epsilon_{3}} \in \kappa(\eta)^{\times}$be the residue class of $\epsilon_{2} \epsilon_{3}=3\left(1-\zeta_{3}\right) t^{-3 e^{\prime}} \in A_{\eta}^{\times}$, which in fact belongs to $\mathbb{F}^{\times}$by (5.3.4). We note that $3 \mid e^{\prime}$ and that $\operatorname{ch}(\eta)=3$. By Sublemma 5.3.9 (3) and (5.3.7), the first term (resp. the second term) of the sum of symbols in question maps to

$$
\overline{\epsilon_{2} \epsilon_{3}} \cdot \frac{\left(2 y^{2} z+y z^{2}\right) d y+\left(2 y^{2} z+y^{3}\right) d z}{z^{2}} \quad\left(\operatorname{resp} . \overline{\epsilon_{2} \epsilon_{3}} \cdot \frac{z(y+z)^{2} d y-y(y+z)^{2} d z}{z^{2}}\right)
$$

under the Bloch-Kato isomorphism (cf. (2.3.4) and (2.6.3) $)$. Since $\operatorname{ch}(\eta)=3$, the sum of these 1-forms agrees with $\overline{\epsilon_{2} \epsilon_{3}}(z d y-y d z)$.

(2) Noting that $e^{\prime} \geq 3$ and that $H$ is a 3 -torsion, we have

$$
\begin{aligned}
& \left\{1+t^{3 e-3} \epsilon_{3} y^{2} z, 1+t^{e^{\prime}} \frac{\epsilon_{2} y}{z}\right\} \\
& \equiv-\left\{1+t^{3 e^{\prime}-3} \epsilon_{2} \epsilon_{3} y^{3},-t^{3 e-3} \epsilon_{3} y^{2} z\right\} \bmod U^{4 e^{\prime}-3} H \quad \text { (Sublemma 5.3.9 (2), (3)) } \\
& =-\left\{1+\left(\zeta_{3}-1\right)^{3} t^{-3} y^{3}\left(1+\left(\zeta_{3}-1\right)\right)^{2}, \epsilon_{3} y^{2} z\right\} \quad\left(t^{3 e^{\prime}} \epsilon_{2} \epsilon_{3}=\left(\zeta_{3}-1\right)^{3} \zeta_{3}^{2}\right) \\
& \equiv-\left\{1+\left(\zeta_{3}-1\right)^{3} t^{-3} y^{3}, \epsilon_{3} y^{2} z\right\} \bmod U^{4 e^{\prime}-3} H \quad \text { (Sublemma 5.3.9(2)) } \\
& =\left\{\frac{\left(1+\left(\zeta_{3}-1\right) t^{-1} y\right)^{3}}{1+\left(\zeta_{3}-1\right)^{3} t^{-3} y^{3}}, \epsilon_{3} y^{2} z\right\} \\
& =\left\{1+\frac{3\left(\zeta_{3}-1\right) t^{-1}\left(y+\left(\zeta_{3}-1\right) t^{-1} y^{2}\right)}{1+\left(\zeta_{3}-1\right)^{3} t^{-3} y^{3}}, \epsilon_{3} y^{2} z\right\} \quad \in U^{3 e^{\prime}-1} H \text {. }
\end{aligned}
$$


(3) Similarly as for (2), we have

$$
\begin{aligned}
& \left\{1+t^{3 e-3} \epsilon_{3} y z^{2}, 1+t^{e^{\prime}} \frac{\epsilon_{2} y}{z}\right\} \\
& \equiv-\left\{1+t^{3 e^{\prime}-3} \epsilon_{2} \epsilon_{3} y^{2} z,-t^{3 e-3} \epsilon_{3} y z^{2}\right\} \quad \bmod U^{4 e^{\prime}-3} H \quad \text { (Sublemma5.3.9 (2), (3)) } \\
& =-\left\{1+t^{3 e^{\prime}-3} \epsilon_{2} \epsilon_{3} y^{2} z, t^{5 e^{\prime}-6} y^{3} z^{3} \epsilon_{2} \epsilon_{3}\right\} \\
& =\left\{1+t^{3 e^{\prime}-3} \epsilon_{2} \epsilon_{3} y^{2} z,\left(1-\zeta_{3}\right) \zeta_{3}^{2}\right\} \\
& =\left\{1+t^{3 e^{\prime}-3} \epsilon_{2} \epsilon_{3} y^{2} z, \epsilon_{4}\left(1+\pi^{e-1} u\right)^{\frac{e}{2}} t^{e^{\prime}} \zeta_{3}^{2}\right\} \\
& \equiv\left\{1+t^{3 e^{\prime}-3} \epsilon_{2} \epsilon_{3} y^{2} z, \epsilon_{4} \zeta_{3}^{2}\right\} \bmod U^{5 e^{\prime}-6} H \\
& \text { (Steinberg relation) } \\
& \left(t^{5 e^{\prime}} \epsilon_{2} \epsilon_{3}^{2}=\left(1-\zeta_{3}\right)^{5} \zeta_{3}^{4}\right) \\
& \left(\epsilon_{4}:=\left(1-\zeta_{3}\right) \pi^{-\frac{e}{2}},(5.3 .4)\right) \\
& \text { (Sublemma 5.3.9(3)). }
\end{aligned}
$$

The last term is contained in $U^{3 e^{\prime}} H$, because $\epsilon_{4} \zeta_{3}^{2}$ belongs to $\mathfrak{o}_{k}^{\times}$and $\mathbb{F}^{\times}$is 3 -divisible. This completes the proof of Lemmas 5.3.10 and 5.3.6(1).

We next prove Lemma 5.3.6(2). Put $\epsilon_{3}:=3 t^{-3 e} \in A_{\eta}^{\times}$. Since

$$
g=\frac{x+z}{x+y}=\frac{y}{z} \cdot \frac{z(t-y)}{y(t-z)}=\frac{y}{z} \cdot\left(1+\frac{t(z-y)}{y(t-z)}\right),
$$

we have

$$
\begin{aligned}
\{1+ & \left.\pi^{e-1} u, g\right\}=\left\{1+\pi^{e-1} \epsilon_{1}\left(y^{2} z+y z^{2}+t v\right), \frac{y}{z} \cdot\left(1+\frac{t(z-y)}{y(t-z)}\right)\right\} \\
\equiv & \left\{1+t^{3 e-2} \epsilon_{3} v, \frac{y}{z}\right\}+\left\{1+t^{3 e-3} \epsilon_{3}\left(y^{2} z+y z^{2}\right), 1+\frac{t(z-y)}{y(t-z)}\right\} \\
& +\left\{1+t^{3 e-3} \epsilon_{3}\left(y^{2} z+y z^{2}\right), \frac{y}{z}\right\}
\end{aligned}
$$

where the congruity holds modulo $U^{3 e-1} H$ by Sublemma 5.3.9 (1)-(3) and (5.3.4). One can easily check that the last term belongs to $U^{3 e} H$ by similar computations as in Lemma 5.3.10(3). One can also check that the sum of symbols

$$
\left\{1+t^{3 e-2} \epsilon_{3} v, \frac{y}{z}\right\}+\left\{1+t^{3 e-3} \epsilon_{3}\left(y^{2} z+y z^{2}\right), 1+\frac{t(z-y)}{y(t-z)}\right\} \in U^{3 e-2} H
$$

maps to $\overline{\epsilon_{3}}(y d z-z d y)$ under the Bloch-Kato isomorphism $\operatorname{gr}_{U}^{3 e-2} H \simeq \Omega_{\eta}^{1}$ (with respect to the prime element $t \in A_{\eta}$ ), by Sublemma 5.3.9 (3) and (5.3.7). Here $\overline{\epsilon_{3}} \in \kappa(\eta)^{\times}$denotes the residue class of $\epsilon_{3}=3 t^{-3 e}$, which belongs to $\mathbb{F}^{\times}$by (5.3.4). This completes the proof of Lemma 5.3.6 (2), Proposition 5.3.5 and Theorem 5.1.1(2). 


\section{Proof of Theorem 1.1 .3}

In this section, we prove Theorem 1.1.3. Let $k$ be $p$-adic local field, and let $\mathfrak{o}_{k}$ be the integer ring of $k$. Put $S:=\operatorname{Spec}\left(\mathfrak{o}_{k}\right)$. Let $\mathscr{X}$ be a regular scheme which is proper flat of finite type over $S$. Let $Y$ be the closed fiber of $\mathscr{X} / S$.

\subsection{Proof of Theorem 1.1.3(1). Let}

$$
\langle,\rangle: \mathrm{CH}_{0}(X) \times \operatorname{Br}(X) \longrightarrow \mathbb{Q} / \mathbb{Z}
$$

be the Brauer-Manin pairing $(\mathbf{M})$ in the introduction, and let $\omega$ be an element of $\operatorname{Br}(X)$ with $\langle c, \omega\rangle=0$ for any $c \in \mathrm{CH}_{0}(X)$. It suffices to show that $\omega$ belongs to $\operatorname{Br}(\mathscr{X})$. Indeed, $\omega$ is 0-unramified in the sense of Definition 4.1.1(2), and the assertion follows from Corollary 4.2.3, where we have assumed the purity of Brauer groups for $\mathscr{X}$. This completes the proof.

6.2. Proof of Theorem 1.1.3(2). We start the proof of Theorem 1.1.3(2), which will be finished in 6.3 below. Let us recall the map (1.1.2), which we denote by $\Phi$ in what follows:

$$
\Phi: A_{0}(X) \longrightarrow \operatorname{Hom}(\operatorname{Br}(X) / \operatorname{Br}(k)+\operatorname{Br}(\mathscr{X}), \mathbb{Q} / \mathbb{Z})
$$

By Theorem 1.1.3(1), $\Phi$ has dense image. To prove that $\Phi$ is surjective, it is enough to show

$$
\operatorname{Im}(\Phi) \simeq \mathbb{Z}_{p}^{\oplus r} \oplus T
$$

for some non-negative integer $r$ and some finite group $T$. We are thus reduced to the following proposition, where we do not assume the purity of Brauer groups:

Proposition 6.2.1. Put $B:=\operatorname{Br}(X) / \operatorname{Br}(k)+\operatorname{Br}(\mathscr{X})$ and $D_{\ell}:=\operatorname{Hom}\left(B_{\ell \text {-tors }}, \mathbb{Q} / \mathbb{Z}\right)$ for $a$ prime number $\ell$. Then:

(1) $D_{\ell}$ is finitely generated over $\mathbb{Z}_{\ell}$ for any $\ell$.

(2) If $\ell \neq p$, then $D_{\ell}$ is finite and the map $\Phi_{\ell}: A_{0}(X) \rightarrow D_{\ell}$ induced by $\Phi$ is surjective. The image of $\Phi_{p}: A_{0}(X) \rightarrow D_{p}$ is a $\mathbb{Z}_{p}$-submodule of $D_{p}$.

(3) $D_{\ell}$ is zero for almost all $\ell \neq p$.

Remark 6.2.2. The ' $\ell \neq p$ ' case of Proposition 6.2.1(2) is proved in [CTS], Corollaire 2.6.

Proof of Proposition 6.2.1. The assertion (1) is obvious. We prove (2). Note that the map $\Phi_{\ell}$ with $\ell \neq p$ has dense image with respect to the $\ell$-adic topology on $D_{\ell}$ (by the $\ell$-primary part of Theorem 1.1.3(1) and the absolute purity). By a standard norm argument, we may suppose that $X$ has a $k$-rational point. Then we have a surjective map

$$
\bigoplus_{C} A_{0}(C) \longrightarrow A_{0}(X) \text {. }
$$

Here $C$ ranges over the smooth integral curves over $k$ which are finite over $X$. Since $k$ is a $p$-adic field by assumption, we have

$$
A_{0}(C) \simeq \mathbb{Z}_{p}^{\oplus r_{C}} \oplus T_{C}
$$

for a non-negative integer $r_{C}$ and a finite group $T_{C}$ by a theorem of Mattuck [Ma]. In case $\ell \neq p$, these facts and (1) imply that $\operatorname{Im}\left(\Phi_{\ell}\right)$ is finite and dense, so that $\Phi_{\ell}$ is surjective. To prove the assertion for $\Phi_{p}$, we need to show that the composite map

$$
\mathbb{Z}_{p}^{\oplus r_{C}} \longrightarrow A_{0}(C) \longrightarrow A_{0}(X) \longrightarrow D_{p}
$$


is a homomorphism of $\mathbb{Z}_{p}$-modules, that is, continuous with respect to the $p$-adic topology. Let $f_{C}: \operatorname{Br}(k) \rightarrow \operatorname{Br}(C)$ be the natural restriction map. Since the above composite map factors through the map

$$
\mathbb{Z}_{p}^{\oplus r_{C}} \hookrightarrow A_{0}(C) \longrightarrow \operatorname{Hom}\left(\operatorname{Coker}\left(f_{C}\right)_{p \text {-tors }}, \mathbb{Q} / \mathbb{Z}\right),
$$

it suffices to see the continuity of this map, which is a consequence of [S1], Theorem (9.2). Thus we obtain Proposition 6.2.1(2).

We next show Proposition 6.2.1(3). For $\ell \neq p$, we have $\operatorname{Im}\left(\Phi_{\ell}\right)=D_{\ell}$ as we mentioned in the proof of (2). On the other hand, we have

$$
\operatorname{Im}\left(\Phi_{\ell}\right) \simeq \operatorname{Im}\left(A_{0}(X) \rightarrow H^{2 N}\left(X, \mathbb{Z}_{\ell}(N)\right)\right)
$$

with $N:=\operatorname{dim}(X)$ by the Poincaré duality. Therefore by the alteration theorem of de Jong $[\mathrm{dJ}]$ and a standard norm argument using the functoriality of cycle class maps, the assertion is reduced to the case that $\mathscr{X}$ has strict semistable reduction over $S$ where 'strict' means that all irreducible components of $Y$ are smooth. We prove that $\bigoplus_{\ell \neq p} D_{\ell}$ is finite, assuming that $\mathscr{X} / S$ has strict semistable reduction. For a torsion abelian group $M$, let $M^{\prime}$ be its prime-to- $p$ part. For $n \in \mathbb{Z}$, let $\mathbb{Q} / \mathbb{Z}^{\prime}(n)$ be the étale sheaf $\bigoplus_{\ell \neq p} \mathbb{Q}_{\ell} / \mathbb{Z}_{\ell}(n)$. Consider a commutative diagram with exact rows

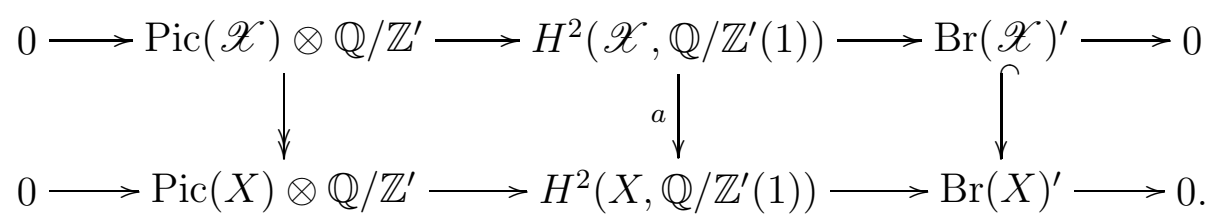

Since the left vertical arrow is surjective, we have

$$
\operatorname{Br}(X)^{\prime} / \operatorname{Br}(\mathscr{X})^{\prime} \simeq \operatorname{Coker}(a) \simeq \operatorname{Ker}\left(H_{Y}^{3}\left(\mathscr{X}, \mathbb{Q} / \mathbb{Z}^{\prime}(1)\right) \rightarrow H^{3}\left(\mathscr{X}, \mathbb{Q} / \mathbb{Z}^{\prime}(1)\right)\right) .
$$

Our task is to show that the complex

$$
\operatorname{Br}(k)^{\prime} \longrightarrow H_{Y}^{3}\left(\mathscr{X}, \mathbb{Q} / \mathbb{Z}^{\prime}(1)\right) \longrightarrow H^{3}\left(\mathscr{X}, \mathbb{Q} / \mathbb{Z}^{\prime}(1)\right)
$$

has finite cohomology group at the middle. Let $k^{\text {ur }}$ be the maximal unramified extension of $k$, and put $X^{\text {ur }}:=\mathscr{X} \otimes_{\mathfrak{o}_{k}} \mathfrak{o}_{k^{\text {ur }}}$ and $\bar{Y}:=Y \otimes_{\mathbb{F}} \overline{\mathbb{F}}$. In view of the short exact sequences

$$
\begin{aligned}
& 0 \rightarrow H^{1}\left(\mathbb{F}, H_{\bar{Y}}^{2}\left(X^{\text {ur }}, \mathbb{Q} / \mathbb{Z}^{\prime}(1)\right)\right) \rightarrow H_{Y}^{3}\left(\mathscr{X}, \mathbb{Q} / \mathbb{Z}^{\prime}(1)\right) \rightarrow H_{\bar{Y}}^{3}\left(X^{\text {ur }}, \mathbb{Q} / \mathbb{Z}^{\prime}(1)\right)^{G_{\mathbb{F}}} \rightarrow 0, \\
& 0 \rightarrow H^{1}\left(\mathbb{F}, H^{2}\left(X^{\text {ur }}, \mathbb{Q} / \mathbb{Z}^{\prime}(1)\right)\right) \rightarrow H^{3}\left(\mathscr{X}, \mathbb{Q} / \mathbb{Z}^{\prime}(1)\right) \rightarrow H^{3}\left(X^{\text {ur }}, \mathbb{Q} / \mathbb{Z}^{\prime}(1)\right)^{G_{\mathbb{F}}} \rightarrow 0
\end{aligned}
$$

obtained from Hochschild-Serre spectral sequences and the fact $\operatorname{cd}(\mathbb{F})=1$, we are reduced to the following lemma:

Lemma 6.2.4. (1) The group $H_{\bar{Y}}^{3}\left(X^{\mathrm{ur}}, \mathbb{Q} / \mathbb{Z}^{\prime}(1)\right)^{G_{\mathbb{F}}}$ is finite.

(2) The composite map $\operatorname{Br}(k)^{\prime} \rightarrow H_{Y}^{3}\left(\mathscr{X}, \mathbb{Q} / \mathbb{Z}^{\prime}(1)\right) \rightarrow H_{\bar{Y}}^{3}\left(X^{\mathrm{ur}}, \mathbb{Q} / \mathbb{Z}^{\prime}(1)\right)^{G_{\mathbb{F}}}$ is zero. Consequently, the complex (6.2.3) induces a complex

$$
\operatorname{Br}(k)^{\prime} \longrightarrow H^{1}\left(\mathbb{F}, H_{\bar{Y}}^{2}\left(X^{\mathrm{ur}}, \mathbb{Q} / \mathbb{Z}^{\prime}(1)\right)\right) \longrightarrow H^{1}\left(\mathbb{F}, H^{2}\left(X^{\mathrm{ur}}, \mathbb{Q} / \mathbb{Z}^{\prime}(1)\right)\right),
$$

(3) The complex (6.2.5) has finite cohomology group at the middle. 
We first show Lemma 6.2.4(1). For $q \geq 1$, let $\bar{Y}^{(q)}$ be the disjoint union of the intersections of $q$ distinct irreducible components of $\bar{Y}$. Note that all connected components of $\bar{Y}^{(q)}$ are smooth proper varieties over $\overline{\mathbb{F}}$ of $\operatorname{dimension} \operatorname{dim}(\mathscr{X})-q$. By the Mayer-Vietoris spectral sequence

$$
E_{1}^{u, v}=H^{2 u+v-2}\left(\bar{Y}^{(-u+1)}, \mathbb{Q} / \mathbb{Z}^{\prime}(u)\right) \Longrightarrow H_{\bar{Y}}^{u+v}\left(X^{\mathrm{ur}}, \mathbb{Q} / \mathbb{Z}^{\prime}(1)\right)
$$

(cf. [RZ], Satz 2.21), we have the following exact sequence of $G_{\mathbb{F}}$-modules:

$$
0 \longrightarrow H^{1}\left(\bar{Y}^{(1)}, \mathbb{Q} / \mathbb{Z}^{\prime}\right) \longrightarrow H_{\bar{Y}}^{3}\left(X^{\mathrm{ur}}, \mathbb{Q} / \mathbb{Z}^{\prime}(1)\right) \longrightarrow H^{0}\left(\bar{Y}^{(2)}, \mathbb{Q} / \mathbb{Z}^{\prime}(-1)\right) .
$$

Hence the assertion follows from the finiteness of $H^{1}\left(\bar{Y}^{(1)}, \mathbb{Q} / \mathbb{Z}^{\prime}\right)^{G_{\mathbb{F}}}$ due to Katz-Lang [KL]. Lemma 6.2.4 (2) is a consequence of (1) and the fact that $\operatorname{Br}(k)^{\prime}$ is divisible.

We next show Lemma 6.2.4(3). For an abelian group $M$, let $M_{\mathbb{L} \text {-div }}$ be the subgroup of elements which are divisible in $M$ by all integers prime to $p$, that is,

$$
M_{\mathbb{L}-\text { div }}:=\bigcap_{n \in \mathbb{Z},(n, p)=1} n \cdot M \text {. }
$$

We define the discrete $G_{\mathbb{F}}$-module $\Xi$ as $\operatorname{Pic}\left(X^{\text {ur }}\right) / \operatorname{Pic}\left(X^{\text {ur }}\right)_{\mathbb{L} \text {-div }}$. For this group we will prove:

Lemma 6.2.7. (1) $\Xi$ is a finitely generated abelian group.

(2) For any positive integer $n$ prime to $p,{ }_{n} \mathrm{Pic}\left(X^{\mathrm{ur}}\right)$ is finite. Consequently, $\operatorname{Pic}\left(X^{\mathrm{ur}}\right)_{\mathbb{L} \text {-div }}$ is divisible by integers prime to $p(c f .[\bar{J}], \S 4)$, and we have a natural injective map

$$
\alpha: \Xi \otimes \mathbb{Q} / \mathbb{Z}^{\prime} \longrightarrow H^{2}\left(X^{\mathrm{ur}}, \mathbb{Q} / \mathbb{Z}^{\prime}(1)\right) .
$$

(3) The map

$$
\alpha^{\prime}: H^{1}\left(\mathbb{F}, \Xi \otimes \mathbb{Q} / \mathbb{Z}^{\prime}\right) \longrightarrow H^{1}\left(\mathbb{F}, H^{2}\left(X^{\mathrm{ur}}, \mathbb{Q} / \mathbb{Z}^{\prime}(1)\right)\right)
$$

induced by $\alpha$ has finite kernel.

The proof of this lemma will be given in $\$ 6.3$ below. We finish our proof of Lemma $6.2 .4(3)$ (and Proposition 6.2.1(3)), admitting this lemma. By Lemma 6.2.7(1), we have the following sequence of finitely generated abelian groups with equivariant $G_{\mathbb{F}}$-action:

$$
\mathbb{Z} \stackrel{\text { diagonal }}{\longrightarrow} \bigoplus_{y \in(\bar{Y})^{0}} \mathbb{Z} \stackrel{g}{\longrightarrow} \Xi
$$

where $g$ is induced by the Gysin map $\bigoplus_{y \in(\bar{Y})^{0}} \mathbb{Z} \rightarrow \operatorname{Pic}\left(X^{\text {ur }}\right)$. This sequence is a complex by the semistability of $\mathscr{X} / S$. Because the $\ell$-primary part of the complex (6.2.5) has finite cohomology group for any $\ell \neq p$ by Proposition 6.2.1(2), one can easily check that the complex (6.2.8) has finite cohomology group as well (in fact, (6.2.8) is exact because the cokernel of the first diagonal map is torsion-free). Hence the cohomology group of the induced complex

$$
\mathbb{Q} / \mathbb{Z} \stackrel{\text { diagonal }}{\longrightarrow} \bigoplus_{y \in(\bar{Y})^{0}} \mathbb{Q} / \mathbb{Z} \stackrel{g \otimes \mathbb{Q} / \mathbb{Z}}{\longrightarrow} \Xi \otimes \mathbb{Q} / \mathbb{Z},
$$

is finite and its order is the same as that of

$$
\operatorname{Coker}\left(g: \bigoplus_{y \in(\bar{Y})^{0}} \mathbb{Z} \longrightarrow \Xi / \Xi_{\text {tors }}\right)_{\text {tors }} \text {. }
$$


We denote its order by $b$. Now we show that the complex (6.2.5) has finite cohomology group. By a standard norm argument, we may suppose that $G_{\mathbb{F}}$ acts trivially on the groups in (6.2.8). Then consider a commutative diagram of complexes

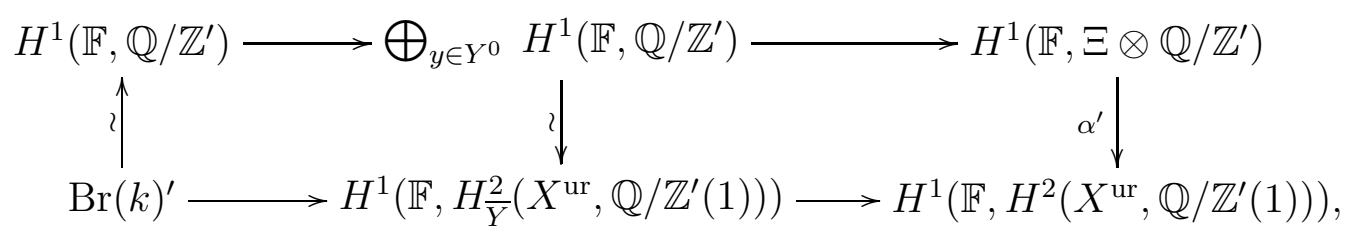

where the upper row is induced by (6.2.9), the lower row is the complex (6.2.5) in question and the bijectivity of the central vertical arrow is obtained from the spectral sequence (6.2.6); the commutativity of the left square follows from the semistability of $\mathscr{X} / S$. The upper row has finite cohomology group of order $b$ up to a power of $p$, and the right vertical arrow $\alpha^{\prime}$ has finite kernel by Lemma 6.2.7(3). Hence the lower row has finite cohomology group of order dividing $b \cdot \#\left(\operatorname{Ker}\left(\alpha^{\prime}\right)\right)$. This completes the proof of Lemma 6.2.4, Proposition 6.2.1 and Theorem 1.1.3 (2), assuming Lemma 6.2.7

6.3. Proof of Lemma 6.2.7. We first prove (1). We change the notation slightly, and put $\Xi(Z):=\operatorname{Pic}(Z) / \operatorname{Pic}(Z)_{\mathbb{L} \text {-div }}$ for a scheme $Z$. For a smooth variety $Z$ over a field, let $\operatorname{NS}(Z)$ be the Néron-Severi group of $Z$. The natural map $\Xi\left(X^{\text {ur }}\right) \rightarrow \Xi(\bar{Y})$ is injective by the proper base-change theorem:

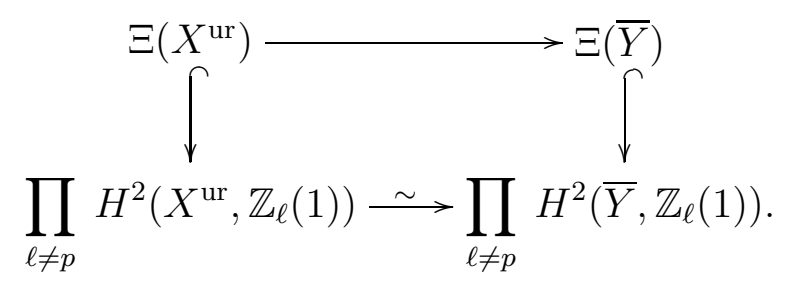

We show that $\Xi(\bar{Y})$ is finitely generated. Since $Y$ has simple normal crossings on $\mathscr{X}$, we have the following exact sequence of sheaves on $Y_{\text {ét }}$ :

$$
0 \longrightarrow \mathscr{O}_{Y}^{\times} \longrightarrow \mathbb{G}_{\mathrm{m}, Y^{(1)}} \longrightarrow \mathbb{G}_{\mathrm{m}, Y^{(2)}} \longrightarrow \cdots
$$

where $Y^{(1)}(q \geq 1)$ is as in the proof of Lemma 6.2.4(1). By this exact sequence, it is easy to see that the kernel of the natural map $\operatorname{Pic}(\bar{Y}) \rightarrow \operatorname{Pic}\left(\bar{Y}^{(1)}\right)$ is an extension of a finite group by a torsion divisible group. Hence the induced map

$$
\Xi(\bar{Y}) \longrightarrow \Xi\left(\bar{Y}^{(1)}\right) \simeq \mathrm{NS}\left(\bar{Y}^{(1)}\right) / \mathrm{NS}\left(\bar{Y}^{(1)}\right)_{p \text {-tors }}
$$

has finite kernel, and the assertion follows from the fact that the last group is a finitely generated abelian group. Thus we obtain Lemma 6.2.7(1).

Lemma 6.2.7(2) follows from the finiteness of the groups $H^{1}\left(X^{\mathrm{ur}}, \mu_{n}\right)$ with $(n, p)=1$. The details are straight-forward and left to the reader.

We prove Lemma 6.2.7(3). By the proof of (1), the natural map $\Xi\left(X^{\mathrm{ur}}\right) \rightarrow \Xi\left(\bar{Y}^{(1)}\right)$ has finite kernel and $\Xi\left(\bar{Y}^{(1)}\right)$ is finitely generated, which implies that the left vertical arrow in the 
following commutative diagram has finite kernel by a standard norm argument:

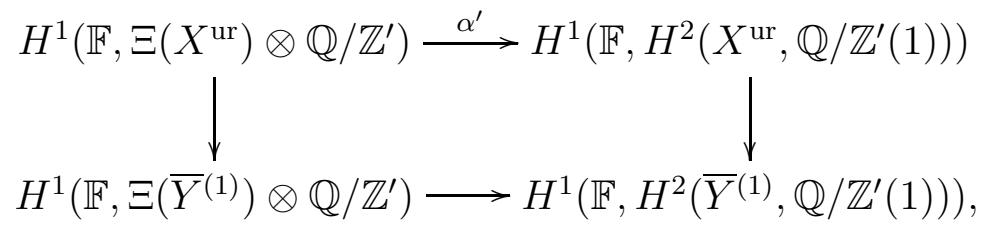

where the bottom horizontal arrow is defined in the same way as $\alpha^{\prime}$ and it has finite kernel by [SS2], Lemma 6.7 (note that $\Xi\left(\bar{Y}^{(1)}\right)=\mathrm{NS}\left(\bar{Y}^{(1)}\right) / \mathrm{NS}\left(\bar{Y}^{(1)}\right)_{p \text {-tors }}$ ). Hence $\alpha^{\prime}$ has finite kernel as well. This completes the proof of Lemma 6.2.7.

6.4. Appendix: Degree of 0-cycles. Let $\mathscr{X}$ be a regular scheme of finite type over $S=$ $\operatorname{Spec}\left(\mathfrak{o}_{k}\right)$. We do not assume the properness of $\mathscr{X} / S$ here. Put $Q:=X_{0}$, and let $Q_{\text {fin }}$ be the subset of $Q$ consisting of all closed points on $X$ whose closure in $\mathscr{X}$ are finite over $S$. If $\mathscr{X}$ is proper over $S$ then we have $Q_{\text {fin }}=Q$. For $y \in Y^{0}$, let $e_{y}$ be the multiplicity of $y$ in $\mathscr{X} \otimes_{\mathfrak{o}_{k}} \mathbb{F}$, and let $f_{y}$ be the degree over $\mathbb{F}$ of the algebraic closure of $\mathbb{F}$ in $\kappa(y)$.

Theorem 6.4.1. Assume that the purity of Brauer groups holds for $\mathscr{X}$. Then the following three numbers are equal to one another:

$N_{1}$ : the order of the kernel of the composite map $\operatorname{Br}(k) \rightarrow \operatorname{Br}(X) \rightarrow \operatorname{Br}(X) / \operatorname{Br}(\mathscr{X})$.

$N_{2}$ : the greatest common measure of the degrees $[\kappa(v): k]$ with $v \in Q_{\mathrm{fin}}$.

$N_{3}$ : the greatest common measure of the integers $e_{y} \cdot f_{y}$ with $y \in Y^{0}$.

Remark 6.4.2. This result was proved by Colliot-Thélène and Saito in [CTS], Théorème 3.1 , up to powers of $p$. However, their arguments work including powers of $p$ after some modification, which we show in what follows.

Proof. By the arguments in [CTS], Théorème 3.1, we have $N_{1}\left|N_{2}\right| N_{3}$. Our task is to show $N_{1}=N_{3}$. Let us consider the composite map

$$
\alpha: \operatorname{Br}(k) \longrightarrow \operatorname{Br}(X) / \operatorname{Br}(\mathscr{X}) \stackrel{\beta}{\longrightarrow} \bigoplus_{y \in Y^{0}} H_{y}^{3}\left(\mathscr{X}, \mathbb{G}_{\mathrm{m}}\right),
$$

where the second map $\beta$ denotes the composite map

$$
\beta: \operatorname{Br}(X) / \operatorname{Br}(\mathscr{X}) \hookrightarrow H_{Y}^{3}\left(\mathscr{X}, \mathbb{G}_{\mathrm{m}}\right) \longrightarrow \bigoplus_{y \in Y^{0}} H_{y}^{3}\left(\mathscr{X}, \mathbb{G}_{\mathrm{m}}\right)
$$

and the last map is injective by the purity assumption on Brauer groups (cf. Remark 4.1.2(3)). Hence it suffices to show the following lemma:

Lemma 6.4.3. Let $\eta$ be a generic point of $Y$. Then the kernel of the composite map

$$
\alpha_{\eta}: \operatorname{Br}(k) \stackrel{\alpha}{\longrightarrow} \bigoplus_{y \in Y^{0}} H_{y}^{3}\left(\mathscr{X}, \mathbb{G}_{\mathrm{m}}\right) \longrightarrow H_{\eta}^{3}\left(\mathscr{X}, \mathbb{G}_{\mathrm{m}}\right)
$$

is isomorphic to $\mathbb{Z} / e_{\eta} f_{\eta} \mathbb{Z}$. 
Proof of Lemma 6.4.3 Let $A_{\eta}$ (resp. $A_{\bar{\eta}}$ ) be the henselization (resp. strict henselization) of $\mathscr{O}_{\mathscr{X}, \eta}$ at its maximal ideal, and let $G_{\eta}$ be the absolute Galois group of $\kappa(\eta)$. By the HochschildSerre spectral sequence

$$
E_{2}^{u, v}=H_{\text {Gal }}^{u}\left(G_{\eta}, H_{\bar{\eta}}^{v}\left(A_{\bar{\eta}}, \mathbb{G}_{\mathrm{m}}\right)\right) \Longrightarrow H_{\eta}^{u+v}\left(A_{\eta}, \mathbb{G}_{\mathrm{m}}\right)\left(=H_{\eta}^{u+v}\left(\mathscr{X}, \mathbb{G}_{\mathrm{m}}\right)\right)
$$

and the standard purity (2.2.3), we have the following exact sequence:

$$
0 \longrightarrow H^{1}(\eta, \mathbb{Q} / \mathbb{Z}) \longrightarrow H_{\eta}^{3}\left(A_{\eta}, \mathbb{G}_{\mathrm{m}}\right) \longrightarrow H_{\bar{\eta}}^{3}\left(A_{\bar{\eta}}, \mathbb{G}_{\mathrm{m}}\right)^{G_{\eta}}
$$

Next let $s$ be the closed point of $S$, and let $k^{\text {ur }}$ be the maximal unramified extension of $k$. The same computation for $S$ yields an isomorphism $H^{1}(\mathbb{F}, \mathbb{Q} / \mathbb{Z}) \simeq H_{s}^{3}\left(S, \mathbb{G}_{\mathrm{m}}\right)(\simeq \operatorname{Br}(k))$, because we have $H_{\frac{3}{s}}\left(\mathscr{O}_{S, \bar{s}}^{\mathrm{sh}}, \mathbb{G}_{\mathrm{m}}\right) \simeq \operatorname{Br}\left(k^{\text {ur }}\right)=0$ (cf. [Se1], II.3.3). Thus we obtain the following commutative diagram with exact rows:

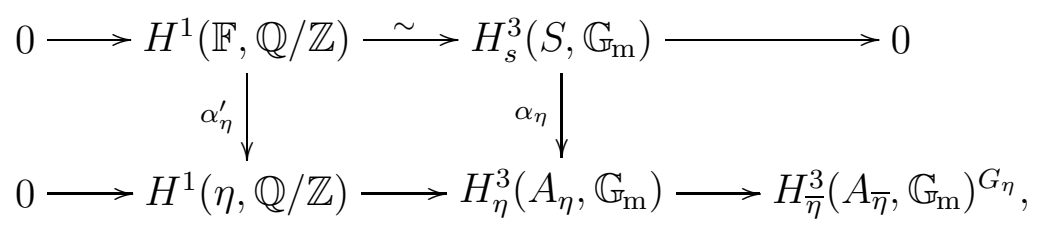

where the arrow $\alpha_{\eta}^{\prime}$ is induced by the right square. One can easily show that

$$
\alpha_{\eta}^{\prime}=e_{\eta} \cdot \operatorname{Res}
$$

where Res denotes the natural restriction map $H^{1}(\mathbb{F}, \mathbb{Q} / \mathbb{Z}) \rightarrow H^{1}(\eta, \mathbb{Q} / \mathbb{Z})$ induced by the structure map $\eta \rightarrow \operatorname{Spec}(\mathbb{F})$. Thus we have

$$
\operatorname{Ker}\left(\alpha_{\eta}\right) \simeq \operatorname{Ker}\left(\alpha_{\eta}^{\prime}\right) \simeq \mathbb{Z} / e_{\eta} f_{\eta} \mathbb{Z}
$$

This completes the proof of Lemma 6.4.3 and Theorem 6.4.1. 


\section{Proof of Theorem 1.3 .1}

In this section, we prove Theorem 1.3.1. Let the notation be as in $\$ 1.3$. Put $S:=\operatorname{Spec}\left(\mathfrak{o}_{k}\right)$ and let $Y$ be the divisor on $\mathscr{X}$ defined by the radical of $(p) \subset \mathscr{O}_{\mathscr{X}}$.

7.1. Key diagram. For $v \in X_{0}$, the closure $\overline{\{v\}} \subset \mathscr{X}$ contains exactly one closed point of $Y$ by the properness of $\mathscr{X}$ over $S$.

Definition 7.1.1. We define the map of sets sp : $X_{0} \rightarrow Y_{0}$ by the law that $\operatorname{sp}(v)=x$ if and only if $x$ is the closed point of $\overline{\{v\}} \subset \mathscr{X}$. Since $\mathfrak{o}_{k}$ is henselian local, there is a natural identification of sets

$$
\operatorname{sp}^{-1}(\{x\})=\left\{\operatorname{closed} \text { points on } \operatorname{Spec}\left(\mathscr{O}_{\mathscr{X}, x}^{\mathrm{h}}\left[p^{-1}\right]\right)\right\} \quad\left(x \in Y_{0}\right)
$$

where $\mathscr{O}_{\mathscr{X}, x}^{\mathrm{h}}$ denotes the henselization of $\mathscr{O}_{\mathscr{X}, x}$ at its maximal ideal.

We construct the key diagram (7.1.4) below. Let $U \subset Y$ be a regular dense open subset. For $x \in U_{0}$, put $A_{x}:=\mathscr{O}_{\mathscr{X}, x}^{\mathrm{h}}$ and let $\psi_{x, p^{r}}$ be the composite map

$$
\psi_{x, p^{r}}: p^{r} \operatorname{Br}\left(A_{x}\left[p^{-1}\right]\right) \stackrel{\text { specialization }}{\longrightarrow} \prod_{v \in \mathrm{sp}^{-1}(\{x\})} p^{r} \operatorname{Br}(v) \stackrel{\text { invariant }}{\sim} \prod_{v \in \mathrm{sp}^{-1}(\{x\})} \mathbb{Z} / p^{r} \mathbb{Z} .
$$

We put $Q_{U}:=\mathrm{sp}^{-1}\left(U_{0}\right)$, and define the map

$$
\psi_{p^{r}}: \prod_{x \in U_{0}} p^{r} \operatorname{Br}\left(A_{x}\left[p^{-1}\right]\right) \longrightarrow \prod_{v \in Q_{U}} \mathbb{Z} / p^{r} \mathbb{Z}
$$

as the direct product of $\psi_{x, p^{r}}$ 's with $x \in U_{0}$. Next we construct a canonical map

$$
\theta_{p^{r}}: H_{Y}^{3}\left(\mathscr{X}, \mathfrak{T}_{r}(1)\right) \longrightarrow \prod_{x \in U_{0}} p^{r} \operatorname{Br}\left(A_{x}\left[p^{-1}\right]\right),
$$

where $\mathfrak{T}_{r}(1)=\mathfrak{T}_{r}(1)_{\mathscr{X}}$ denotes the $p$-adic étale Tate twist [Sa2]. For this we will prove

Lemma 7.1.2. Let $x$ be a closed point on $U$ and let $Y_{x}$ be the divisor on $\mathscr{X}_{x}:=\operatorname{Spec}\left(A_{x}\right)$ defined by the radical of $(p) \subset A_{x}$. Then there is a canonical isomorphism

$$
{ }_{p^{r}} \operatorname{Br}\left(A_{x}\left[p^{-1}\right]\right) \simeq H_{Y_{x}}^{3}\left(\mathscr{X}_{x}, \mathfrak{T}_{r}(1)\right) .
$$

By this lemma, we define the desired map $\theta_{p^{r}}$ as the restriction map

$$
H_{Y}^{3}\left(\mathscr{X}, \mathfrak{T}_{r}(1)\right) \longrightarrow \prod_{x \in U_{0}} H_{Y_{x}}^{3}\left(\mathscr{X}_{x}, \mathfrak{T}_{r}(1)\right) \simeq \prod_{x \in U_{0}} p^{r} \operatorname{Br}\left(A_{x}\left[p^{-1}\right]\right)
$$

Proof of Lemma 7.1.2. Consider the localization exact sequence

$$
H^{2}\left(\mathscr{X}_{x}, \mathfrak{T}_{r}(1)\right) \longrightarrow H^{2}\left(\mathscr{X}_{x}\left[p^{-1}\right], \mu_{p^{r}}\right) \longrightarrow H_{Y_{x}}^{3}\left(\mathscr{X}_{x}, \mathfrak{T}_{r}(1)\right) \longrightarrow H^{3}\left(\mathscr{X}_{x}, \mathfrak{T}_{r}(1)\right),
$$

where we have used the property that $\mathfrak{T}_{r}(1)$ is isomorphic to $\mu_{p^{r}}$ outside of characteristic $p$ (cf. [Sa2] $)$. Since $A_{x}\left[p^{-1}\right]$ is a UFD, we have $H^{2}\left(\mathscr{X}_{x}\left[p^{-1}\right], \mu_{p^{r}}\right) \simeq{ }_{p^{r}} \operatorname{Br}\left(A_{x}\left[p^{-1}\right]\right)$. On the other hand, we have

$$
\begin{aligned}
& H^{2}\left(\mathscr{X}_{x}, \mathfrak{T}_{r}(1)\right) \stackrel{(*)}{\simeq}{ }_{p^{r}} \operatorname{Br}\left(\mathscr{X}_{x}\right) \simeq{ }^{r} \operatorname{Br}(x)=0, \\
& H^{3}\left(\mathscr{X}_{x}, \mathfrak{T}_{r}(1)\right) \simeq H^{3}\left(x,\left.\mathfrak{T}_{r}(1)\right|_{x}\right)=0,
\end{aligned}
$$


where the isomorphism $(*)$ follows from the Kummer theory for $\mathscr{O}_{\mathscr{X}}^{\times}$(cf. [Sa2], $§ 4.5$ ), and the last vanishing follows from the facts that $\mathfrak{T}_{r}(1)$ is concentrated in degree 0 and 1 (cf. loc. cit.) and that $\operatorname{cd}(x)=1$. The assertion follows from these facts.

We turn to the proof of Theorem 1.3.1, and define $\alpha$ as the composite map

$$
\alpha: \mathrm{CH}^{d-1}(\mathscr{X}) / p^{r} \stackrel{\varrho_{p^{r}}^{d-1}}{\longrightarrow} H^{2 d-2}\left(\mathscr{X}, \mathfrak{T}_{r}(d-1)\right) \stackrel{\sim}{\longrightarrow} H_{Y}^{3}\left(\mathscr{X}, \mathfrak{T}_{r}(1)\right)^{*},
$$

where for a $\mathbb{Z} / p^{r} \mathbb{Z}$-module $M, M^{*}$ denotes $\operatorname{Hom}\left(M, \mathbb{Z} / p^{r} \mathbb{Z}\right)$, and the last isomorphism follows from the arithmetic duality ([Sa2], Theorem 10.1.1). To prove Theorem[1.3.1, it remains to show the following:

Lemma 7.1.3. (1) The following diagram is commutative:

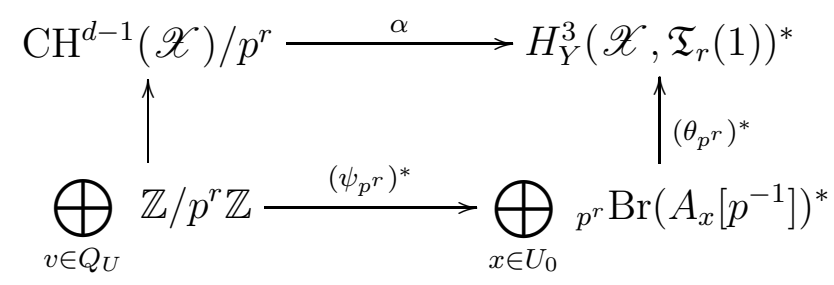

where the left vertical arrow sends $v \in Q_{U}$ to the class of $v$.

(2) The maps $\theta_{p^{r}}$ and $\psi_{p^{r}}$ are injective. Consequently, $\left(\theta_{p^{r}}\right)^{*}$ and $\left(\psi_{p^{r}}\right)^{*}$ are surjective.

We prove (2) in $\$ 7.2$, and then prove (1) in $\$ 7.3$ below.

7.2. Proof of Lemma 7.1.3(2). Put $Z:=Y \backslash U$ and $\mathscr{X}^{\prime}:=\mathscr{X} \backslash Z$. For $x \in U_{0}$, let $\mathscr{X}_{x}$ and $Y_{x}$ be as in Lemma 7.1.2. The map $\psi_{p^{r}}$ is injective by Corollary 3.1.2. Indeed this injectivity is immediately reduced to the case $\zeta_{p} \in k$ by a standard norm argument, because $\mathscr{X}^{\prime}$ is smooth over $S$ by the semistability assumption on $\mathscr{X}$. To show the injectivity of $\theta_{p^{r}}$, we consider the following commutative diagram:

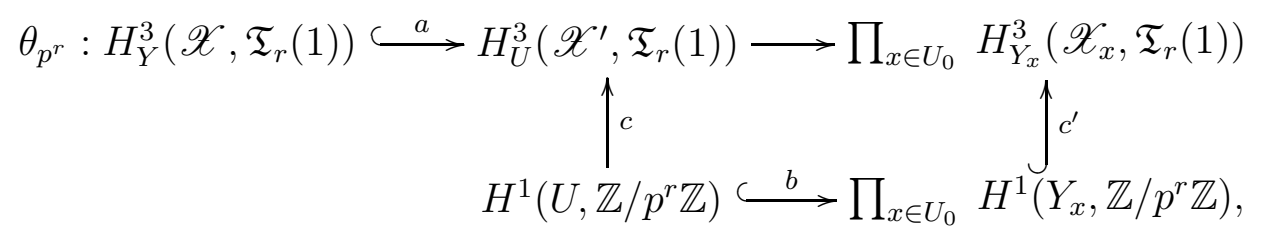

where $a$ and $b$ are restriction maps, and $c$ and $c^{\prime}$ are Gysin maps ([Sa2], Theorem 4.4.7). The arrow $a$ is injective by the purity for $\mathfrak{T}_{r}(1)$ (cf. loc. cit.), and $b$ is injective by the Čebotarev density theorem [ $[\mathrm{Se} 2]$, Theorem 7. The arrow $c^{\prime}$ is injective by Lemma 7.1.2 and Theorem 2.6.2. It remains to show that the induced map Coker $(c) \rightarrow \operatorname{Coker}\left(c^{\prime}\right)$ is injective. Let $i$ be the closed immersion $U \hookrightarrow \mathscr{X}^{\prime}$. Since we have $\operatorname{Coker}(c) \subset H^{0}\left(U, R^{3} i^{!} \mathfrak{T}_{r}(1)\right)$ and

$$
\operatorname{Coker}\left(c^{\prime}\right) \subset \prod_{x \in U_{0}} H^{0}\left(Y_{x},\left.\left(R^{3} i^{\prime} \mathfrak{T}_{r}(1)\right)\right|_{Y_{x}}\right),
$$

it suffices to show that for an étale sheaf $\mathscr{F}$ on a noetherian scheme $W$, the restriction map

$$
H^{0}(W, \mathscr{F}) \longrightarrow \prod_{x \in W_{0}} H^{0}\left(\operatorname{Spec}\left(\mathscr{O}_{W, x}^{\mathrm{h}}\right),\left.\mathscr{F}\right|_{\operatorname{Spec}\left(\mathscr{O}_{W, x}^{\mathrm{h}}\right)}\right)
$$

is injective, which follows from a standard argument using the induction on $\operatorname{dim}(W)$. Thus we obtain Lemma 7.1.3(2). 
7.3. Proof of Lemma 7.1.3(1). Put $\Lambda:=\mathbb{Z} / p^{r} \mathbb{Z}$ and $N:=d-1$ for simplicity. By the definitions of the maps in (7.1.4), it is enough to show that the following diagram commutes for each $v \in Q_{U}$ :

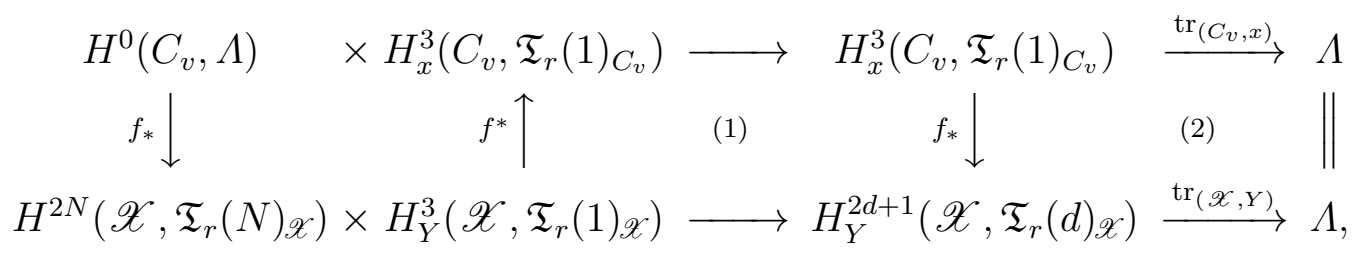

where $C_{v}$ denotes the normalization of $\overline{\{v\}} \subset \mathscr{X}, x$ denotes the closed point of $C_{v}$ and $f$ denotes the canonical finite morphism $C_{v} \rightarrow \mathscr{X}$. See [Sa2], Theorem 10.1.1 for the trace maps. The arrows $f_{*}$ arise from the following relative trace morphism with $n=0,1$ (loc. cit., Theorem 7.1.1):

$$
\operatorname{Gys}_{f}: R f_{*} \mathfrak{T}_{r}(n)_{C_{v}} \longrightarrow \mathfrak{T}_{r}(n+N)_{\mathscr{X}}[2 N] \quad \text { in } D^{b}\left(\mathscr{X}_{\text {ét }}, \Lambda\right),
$$

and the arrow $f^{*}$ arises from the pull-back morphism (loc. cit., Proposition 4.2.8)

$$
\operatorname{res}^{f}: \mathfrak{T}_{r}(1) \mathscr{X}_{*} \longrightarrow R f_{*} \mathfrak{T}_{r}(1)_{C_{v}} \quad \text { in } D^{b}\left(\mathscr{X}_{\text {ét }}, \Lambda\right) .
$$

The commutativity of the square (2) follows from a similar argument as for [Sa2], Lemma 10.2.1. We prove the commutativity (1) of pairings. Consider the following commutative diagram:

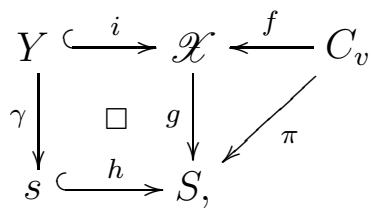

where $s$ denotes the closed point of $S$. Note that we have the base-change isomorphism $R \gamma_{*} R i^{!}=R h^{!} R g_{*}$ by Deligne [SGA4], XVIII.3.1.12.3. To prove the commutativity of pairings in question, it suffices to show that the following diagram commutes in $D^{-}\left(s_{\text {ét }}, \Lambda\right)$ :

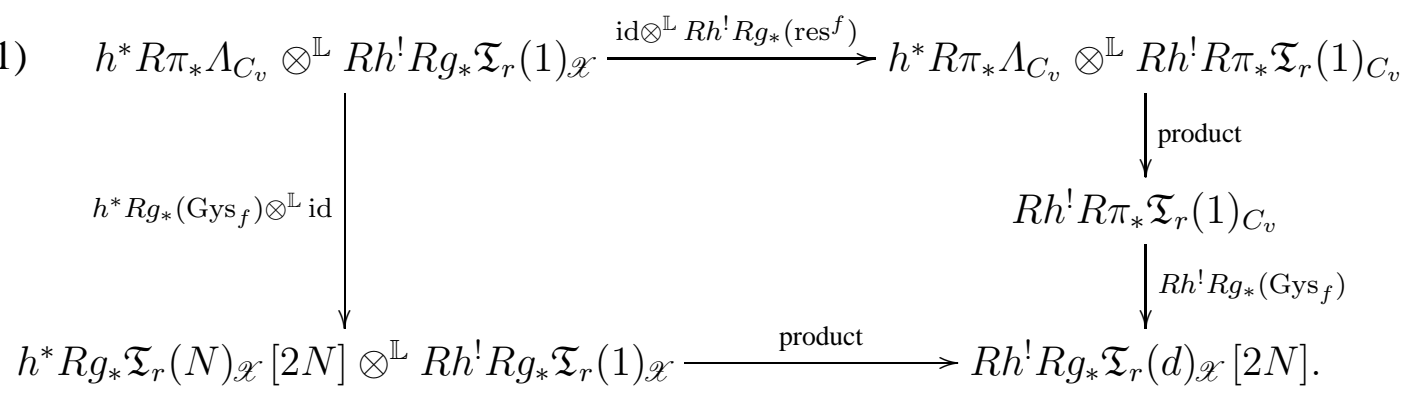

Here the arrows 'product' are induced by the canonical morphism

$$
h^{*} \mathscr{K} \otimes^{\mathbb{L}} R h^{!} \mathscr{L} \longrightarrow R h^{!}\left(\mathscr{K} \otimes^{\mathbb{L}} \mathscr{L}\right) \quad\left(\mathscr{K}, \mathscr{L} \in D^{b}\left(S_{\text {ét }}, \Lambda\right)\right)
$$

and the product structure of Tate twists ([[Sa2], Proposition 4.2.6). We have defined $R h^{!}$for unbounded objects using a result of Spaltenstein $[\mathrm{Sp}]$, Theorem A. Finally one can easily 
check the commutativity of (7.3.1) by applying $R h ! R g_{*}$ to the following commutative diagram in [Sa2], Corollary 7.2.4:

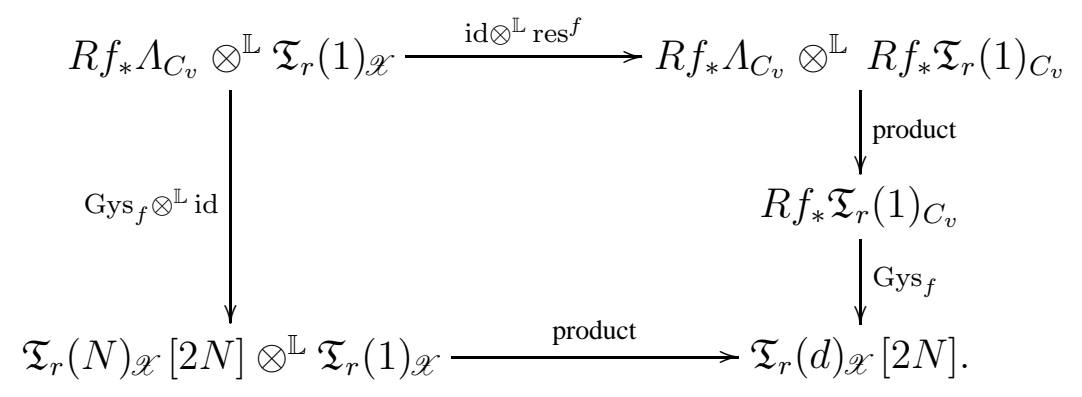

This completes the proof of Lemma 7.1 .3 and Theorem 1.3 .1 .

\section{REFERENCES}

[Be] Beilinson, A. A.: Height pairings between algebraic cycles. In: Manin, Yu. I. (ed.) K-theory, Arithmetic and Geometry, (Lecture Notes in Math. 1289), pp. 1-27, Berlin, Springer, 1987

[B1] Bloch, S.: On the Chow groups of certain rational surfaces. Ann. Sci. Ec. Norm. Sup. (4) 14, 41-59 (1981)

[BK] Bloch, S., Kato, K.: p-adic étale cohomology. Inst. Hautes Études Sci. Publ. Math. 63, 107-152 (1986)

[CT1] Colliot-Thélène, J.-L.: Hilbert's Theorem 90 for $K_{2}$, with application to the Chow groups of rational surfaces. Invent. Math. 71, 1-20 (1983).

[CT2] Colliot-Thélène, J.-L.: Cycles algébriques de torsion et $K$-théorie algébrique. In: Ballico, E. (ed.) Arithmetic Algebraic Geometry, Trento 1991 (Lecture notes in Math. 1553), pp. 1-49, Berlin, 1993

[CT3] Colliot-Thélène, J.-L.: L'arithmétique du groupe de Chow des zéro-cycles. J. Théor. Nombres Bordeaux 7, 51-73 (1995)

[CT4] Colliot-Thélène, J.-L.: Letter to the first author.

[CTOP] Colliot-Thélène, J.-L., Ojanguren, M., Parimala, R.: Quadratic forms over fraction fields of twodimensional henselian rings and Brauer groups of related schemes. In: Parimala, R. (ed.) Algebra, Arithmetic and Geometry, Mumbai 2000, (Tata Inst. Fund. Res. Stud. Math. 16, Vol. I), pp. 185-217, Tata Inst. Fund. Res., Bombay, 2002.

[CTS] Colliot-Thélène, J.-L., Saito, S.: Zéro-cycles sur les variétés $p$-adiques et groupe de Brauer. Internat. Math. Res. Notices 1996, 151-160

[CTSn] Colliot-Thélène, J.-L., Sansuc, J.-J.: On the Chow groups of certain rational surfaces: a sequel to a paper of S. Bloch. Duke Math. J. 48, 421-447 (1981)

[CTSS] Colliot-Thélène, J.-L., Sansuc, J.-J., Soulé, C.: Torsion dans le groupe de Chow de codimension deux. Duke Math. J. 50, 763-801 (1983)

[Da] Dalawat, C. S.: Le groupe de Chow d'une surface rationnelle sur un corps local. Compositio Math. 141, 344-358 (2005)

[dJ] de Jong, A. J.: Smoothness, semi-stability and alterations. Inst. Hautes Études Sci. Publ. Math. 83, 51-93 (1996)

[F] Fujiwara, K.: A proof of the absolute purity conjecture (after Gabber). In: Usui, S., Green, M., Illusie, L., Kato, K., Looijenga, E., Mukai, S., Saito, S. (eds.) Algebraic Geometry, Azumino, 2001, (Adv. Stud. in Pure Math. 36), pp. 153-184, Tokyo, Math. Soc. Japan, 2002

[Ga] Gabber, O.: Some theorems on Azumaya algebras. In: Kervaire, M., Ojanguren, M. (eds.) Groupe de Brauer Séminaire, Les Plans-sur-Bex, 1980, (Lecture Notes in Math. 844), pp. 129-209, Berlin, Springer, 1981

[Gr] Gros, M.: Classes de Chern et classes des cycles en cohomologie logarithmique. Bull. Soc. Math. France Mémoire $\mathrm{N}^{\circ} 21,1985$

[G] Grothendieck, A.: Le groupe de Brauer. In: Dix Exposés sur la Cohomologie des Schémas, pp. 46-188, Amsterdam, North-Holland, 1968 
[H] Hyodo, O.: A note on $p$-adic etale cohomology in the semi-stable reduction case. Invent. Math. 91 543-557 (1988)

[J] Jannsen, U.: Continuous étale cohomology. Math. Ann. 280, 207-245 (1987)

[K] Kato, K.: A generalization of local class field theory by using $K$-groups II. J. Fac. Sci. Univ. of Tokyo Sec. IA 27, 603-683 (1980)

[Kh] Kahn, B.: Descente galoisienne et $K_{2}$ des corps de nombres. $K$-Theory 7, 55-100 (1993)

[KL] Katz, N., Lang, S.: Finiteness theorem for higher geometric class field theory. Enseign. Math. 27, 285-319 (1981)

[L1] Lichtenbaum, S.: Duality theorems for curves over $p$-adic fields. Invent. Math. 7, 120-136 (1969)

[L2] Lichtenbaum, S.: Values of zeta functions at non-negative integers. In: Jager, H. (ed.) Number Theory, Noordwijkerhout, 1983, (Lecture Notes in Math. 1068), pp. 127-138, Berlin, Springer, 1984

[M1] Manin, Yu. I.: Le groupe de Brauer-Grothendieck en géométrie diophantinne. In: Actes du Congrés International des Mathématicients, Nice 1970, Tome 1, pp. 401-411, Paris, Gauthier-Villars, 1971

[M2] Manin, Yu. I.: Cubic forms. Algebra, geometry, arithmetic. Translated by Hazewinkel, M., 2nd ed. (North-Holland Math. Library 4), North-Holland, Amsterdam, 1986

[Ma] Mattuck, A.: Abelian varieties over p-adic ground fields. Ann. of Math. 62, 92-119 (1955)

[MS] Merkur'ev, A. S., Suslin, A. A.: $K$-cohomology of Severi-Brauer varieties and the norm residue homomorphism. Math. USSR Izv. 21, 307-341 (1983)

[Mi] Milne, J. S.: Values of zeta functions of varieties over finite fields. Amer. J. Math. 108, 297-360 (1986)

[PS] Parimala R., Suresh, V.: Zero-cycles on quadratic fibrations: Finiteness theorems and the cycle map. Invent. Math. 122, 83-117 (1995)

[RZ] Rapoport, M., Zink, T.: Über die lokale Zetafunktion von Shimuravarietäten. Monodromiefiltration und verschwindende Zyklen in ungleicher Charakteristik. Invent. Math. 68, 21-101 (1982)

[S1] Saito, S.: Arithmetic on two-dimensional local rings. Invent. Math. 85, 379-414 (1986)

[S2] Saito, S.: On the cycle map for torsion algebraic cycles of codimension two. Invent. Math. 106, 443460 (1991)

[SS1] Saito, S., Sato, K.: A finiteness theorem for zero-cycles over $p$-adic fields. to appear in Ann. of Math.

[SS2] Saito, S., Sato, K.: A p-adic regulator map and finiteness results for arithmetic schemes. preprint, 2009

[Sa1] Sato, K.: Logarithmic Hodge-Witt sheaves on normal crossing varieties. Math. Z. 257, 707-743 (2007)

[Sa2] Sato, K.: p-adic étale Tate twists and arithmetic duality. (with an appendix by Hagihara, K.), Ann. Sci. École Norm. Sup. (4) 40, 519-588 (2007)

[Sch] Schneider, P.: p-adic point of motives. In: Jannsen, U. (ed.) Motives, (Proc. Symp. Pure Math. 55-II), pp. 225-249, Providence, Amer. Math. Soc., 1994

[Se1] Serre, J.-P.: Cohomologie Galoisienne. $5^{e}$ éd., (Lecture Notes in Math. 5), Berlin, Springer, 1992

[Se2] Serre, J.-P.: Zeta and L-functions. In: Schilling, O. F. G. (ed.) Arithmetical Algebraic Geometry, pp. 82-92, New York, Harper and Row, 1965

[Sh] Shiho, A.: On logarithmic Hodge-Witt cohomology of regular schemes. J. Math. Sci. Univ. Tokyo 14, 567-635 (2007)

[Sp] Spaltenstein, N.: Resolutions of unbounded complexes. Compositio Math. 65, 121-154 (1988)

[Sr] Srinivas, V.: Algebraic K-Theory. 2nd ed., (Progr. Math. 90), Boston, Birkhäuser, 1996

[Th] Thomason, R. W.: Absolute cohomological purity. Bull. Soc. Math. France 112, 397-406 (1984)

[Y1] Yamazaki, T.: Formal Chow groups, $p$-divisible groups and syntomic cohomology. Duke Math. J. 102, 359-390 (2000)

[Y2] Yamazaki, T.: On Chow and Brauer groups of a product of Mumford curves. Math. Ann. 333, 549-567 (2005)

[SGA4] Grothendieck, A., Artin, M., Verdier, J.-L., with Deligne, P., Saint-Donat, B.: Théorie des Topos et Cohomologie Étale des Schémas, Tome 3. (Lecture Notes in Math. 305), Berlin, Springer, 1973

\author{
(Shuji Saito) Department of Mathematics, University of Tokyo \\ KomaBA, MEguro-KU, TOKYO 153-8914, JAPAN \\ E-mail address: sshuji@ms.u-tokyo.ac.jp
}

(Kanetomo Sato) Graduate School of Mathematics, Nagoya University 
FURO-CHO, CHIKUSA-KU, NAGOYA 464-8602, JAPAN

E-mail address: kanetomo@math. nagoya-u.ac.jp 